\title{
Statistical analysis of differential white blood cell counts for G-TwYST studies A and C
}

Paul W. Goedhart \& Hilko van der Voet

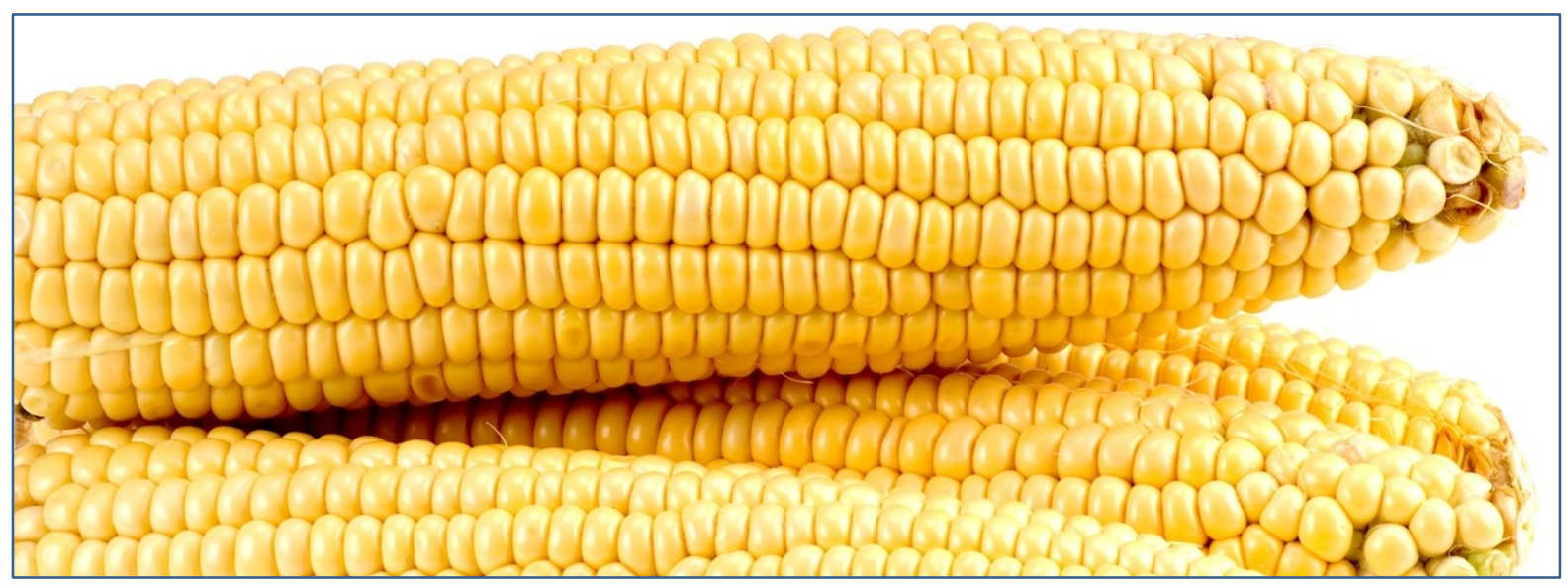

G-TwYST (EU grant agreement no: 632165) Deliverables 4.2a and 4.3e

Biometris report 42.08 .18

August 2018

https://doi.org/10.18174/456855 


\section{Statistical analysis of differential white blood cell counts for G-TwYST studies A and C}

Paul W. Goedhart \& Hilko van der Voet

Biometris, one of the largest groups of quantitative scientists in North-Western Europe, develops statistical and mathematical methods for the quantification of biological processes and processes in our living environment. These methods are applied and validated in practice and are often available as software packages. In addition, we provide education at the undergraduate, Master, PhD, and PostDoc levels, as well as training and consultancy for industry. We cover a wide range of application areas, from gene to ecosystem and from product to production chain. Our goal is to bring quantitative methods to life.

Biometris is the full integration of the chair groups Applied Mathematics (Molenaar) and Applied Statistics (Van Eeuwijk) with the Wageningen Plant Research business unit Biometris (Wehrens).

For more information please visit the website www.biometris.nl or contact:

Biometris, Wageningen University \& Research

P.O. Box 16

6700 AA Wageningen, The Netherlands

Visiting address:

Buildingnumber 107

Droevendaalsesteeg 1, 6708 PD Wageningen, The Netherlands

Phone: +31317480798 or +31317486001

E-mail: biometris@wur.nl 


\section{Contents}

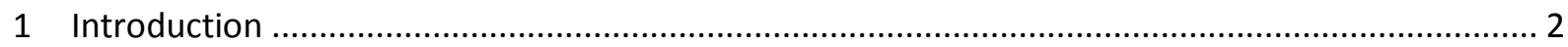

2 Data

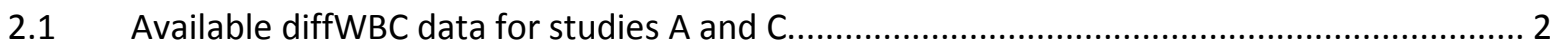

2.2 Outliers and checking of ANOVA assumptions for study A ............................................... 3

2.3 Outliers and checking of ANOVA assumptions for study C .............................................. 12

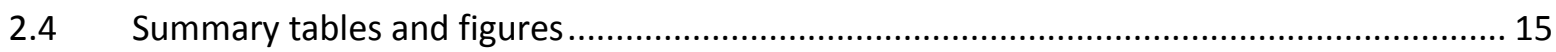

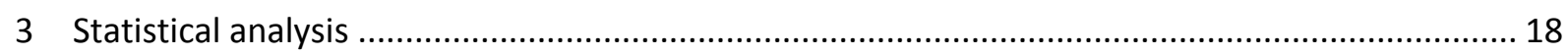

3.1 Equivalence testing for study A using historical data...................................................... 18

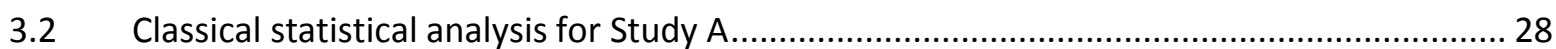

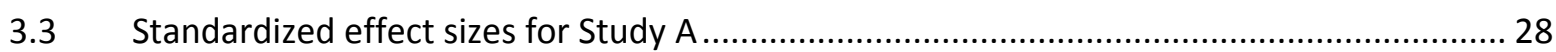

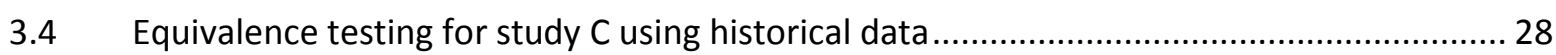

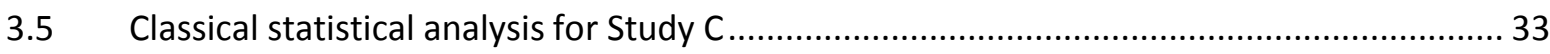

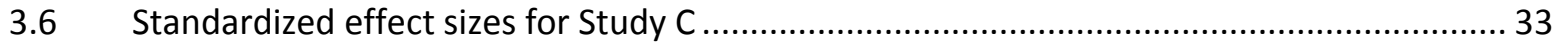

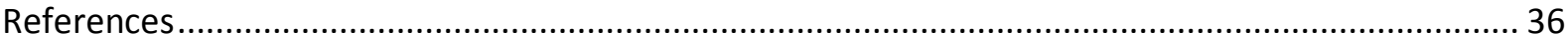

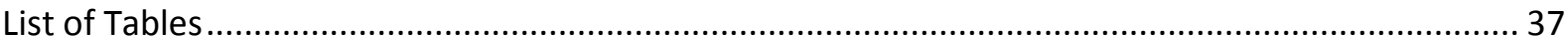

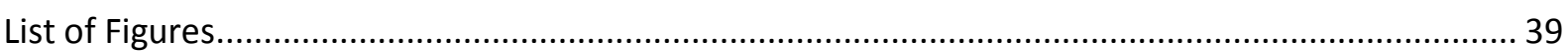

Appendix 1. Graphs of cage means on the original scale for study A ......................................... 41

Appendix 2. Graphs of cage means on the log scale for study A .................................................. 43

Appendix 3. Graphs of cage means on the original scale for study C.......................................... 45

Appendix 4. Graphs of cage means on the log scale for study C................................................ 46

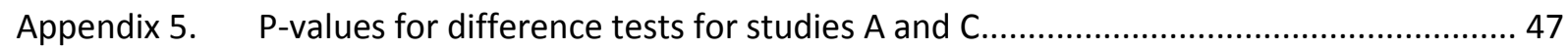

Appendix 6. Tests for normality and homogeneity of variance for studies $A$ and $C$....................... 49 


\section{Introduction}

Statistical analysis of the G-TwYST studies was reported in

Study A: Goedhart \& van der Voet (2018a, 2018b 2018c, 2018d and 2018e)

Study B: Goedhart \& van der Voet (2017)

Study C: Goedhart \& van der Voet (2018f)

Note that for Study A there are separate reports for data acquired after 3, 6, 12 and 24 months as well as a main report. Study B and C were 3 months studies only. These reports lacks a statistical analysis of differential white blood cell counts, or diffWBC for short, for Study A data acquired after 3, 12 and 24 month, and also for study C. This report presents these statistical analysis; it includes, for reasons of completeness, the statistical analysis of diffWBC for study A for 6 months which was already presented in Goedhart \& van der Voet (2018c). The reader is referred to the reports listed above for details of the study plans and the statistical methods used. Here only the results of the statistical analysis are presented.

\section{Data}

\subsection{Available diffWBC data for studies A and C}

The available files for the diffWBC data for studies $A$ and $C$ are given in Table 1 . The number of observations, generally evenly distributed across treatment group, are given in Table 2 . For study $A$ in principle the same rats were observed after 3, 6, 12 and 24 months. However due to mortality, especially after 24 months, additional rats were observed.

Table 1 Data files for G-TwYST studies A and C.

\begin{tabular}{|r|r|r|r|}
\hline Data files differential white blood cell counts Study A & Date & Time & Size (b) \\
\hline 3 months: Diferent WBC GTWYST A-3M database.xlsx & $06-03-2018$ & $12: 53$ & 33,234 \\
\hline 6 months: Differential WBC G-TwYST A - after 6M final 12.12.17.xIsx & $12-12-2017$ & $14: 17$ & 37,026 \\
\hline 12 months: Database Differential WBC GTWYST A - 12M final JT.xlsx & $15-06-2018$ & $10: 40$ & 33,513 \\
\hline 24 months: Database Differential WBC GTWYST A - 24M final JT.xlsx & $14-06-2018$ & $11: 57$ & 30,358 \\
\hline Data file differential white blood cell counts Study C & Date & Time & Size (b) \\
\hline 3 months: Differential WBC - G-TwYST C 20.3.18.xlsx & $20-03-2018$ & $12: 01$ & 28,404 \\
\hline
\end{tabular}

Table 2 Number of animals for which diffWBC data are available in studies A and C.

\begin{tabular}{|c|c|c|}
\hline Variable & Males & Females \\
\hline study A 3 months & 200 & 200 \\
\hline study A 6 months & 200 & 199 \\
\hline study A 12 months & 197 & 202 \\
\hline study A 24 months & 154 & 141 \\
\hline study C & 128 & 128 \\
\hline
\end{tabular}

For each sample 200 cells are classified as a Lymphocyte, Neutrophil, Monocyte, Eosinophil or Basophil. The latter type is rarely found and is not statistically analysed. Occasionally less or more than 200 cell are classified. The data are thus expressed as a percentage of the total number of counted cells. The diffWBC data are thus not independent as the sum of the percentages equals 100 . 


\subsection{Outliers and checking of ANOVA assumptions for study A}

Table 3 and Table 4 lists the five smallest and largest values for every diffWBC response variable in study A for Males and Females respectively. Goedhart \& van der Voet (2018c) found extreme values for Lymphocytes and Neutrophils for female animal 616 in group NK11- in Study A after 6 months. The same animal also had extreme Lymphocytes and Neutrophils values after 3 months, and these are therefore also considered as outliers. Animal 616 died after 372 days and there are no diffWBC data for this animal after 12 and 24 months.

Table 3 The five smallest and five largest observed diffWBC percentages for every response variable observed in study A for Males. Outliers are listed in yellow.

\begin{tabular}{|r|r|r|r|r|r|r|r|}
\hline \multicolumn{5}{|c|}{ Smallest five Lymphocytes Males } & \multicolumn{3}{|c|}{ Largest five Lymphocytes Males } \\
\hline Animal & Cage & Group & Lymphocytes-03 & Animal & Cage & Group & Lymphocytes-03 \\
\hline 266 & 133 & NK33- & 38.0 & 9 & 5 & NK33+ & 85.0 \\
\hline 265 & 133 & NK33- & 45.5 & 35 & 18 & NK33+ & 84.5 \\
\hline 40 & 20 & NK33+ & 50.5 & 161 & 81 & NK11+ & 83.0 \\
\hline 60 & 30 & NK33+ & 52.5 & 231 & 116 & NK33- & 83.0 \\
\hline 195 & 98 & NK11+ & 54.0 & 302 & 151 & Control & 83.0 \\
\hline Animal & Cage & Group & Lymphocytes-06 & Animal & Cage & Group & Lymphocytes-06 \\
\hline 160 & 80 & NK11+ & 54.0 & 22 & 11 & NK33+ & 83.5 \\
\hline 196 & 98 & NK11+ & 54.0 & 300 & 150 & Control & 82.5 \\
\hline 222 & 111 & NK33- & 54.0 & 156 & 78 & NK11+ & 82.0 \\
\hline 269 & 135 & NK33- & 56.5 & 296 & 148 & Control & 81.5 \\
\hline 298 & 149 & Control & 56.5 & 9 & 5 & NK33+ & 81.0 \\
\hline Animal & Cage & Group & Lymphocytes-12 & Animal & Cage & Group & Lymphocytes-12 \\
\hline 153 & 77 & NK11+ & 12.0 & 35 & 18 & NK33+ & 78.5 \\
\hline 195 & 98 & NK11+ & 43.5 & 36 & 18 & NK33+ & 77.0 \\
\hline 81 & 41 & NK11- & 44.0 & 307 & 154 & Control & 75.5 \\
\hline 202 & 101 & NK11+ & 44.0 & 280 & 140 & NK33- & 74.5 \\
\hline 221 & 111 & NK33- & 44.0 & 283 & 142 & Control & 74.5 \\
\hline Animal & Cage & Group & Lymphocytes-24 & Animal & Cage & Group & Lymphocytes-24 \\
\hline 130 & 65 & NK11- & 1.0 & 174 & 87 & NK11+ & 86.5 \\
\hline 123 & 62 & NK11- & 14.0 & 305 & 153 & Control & 83.0 \\
\hline 339 & 170 & Control & 15.5 & 22 & 11 & NK33+ & 81.5 \\
\hline 36 & 18 & NK33+ & 17.0 & 117 & 59 & NK11- & 71.5 \\
\hline 59 & 30 & NK33+ & 18.0 & 200 & 100 & NK11+ & 70.0 \\
\hline
\end{tabular}

\begin{tabular}{|r|r|r|r|r|r|r|r|}
\hline \multicolumn{4}{|c|}{ Smallest five Neutrophils Males } & \multicolumn{4}{|c|}{ Largest five Neutrophils Males } \\
\hline Animal & Cage & Group & Neutrophils-03 & Animal & Cage & Group & Neutrophils-03 \\
\hline 35 & 18 & NK33+ & 11.5 & 266 & 133 & NK33- & 58.0 \\
\hline 79 & 40 & NK11- & 11.5 & 265 & 133 & NK33- & 51.5 \\
\hline 9 & 5 & NK33+ & 13.5 & 40 & 20 & NK33+ & 45.0 \\
\hline 21 & 11 & NK33+ & 14.0 & 60 & 30 & NK33+ & 44.5 \\
\hline 143 & 72 & NK11+ & 14.0 & 195 & 98 & NK11+ & 42.0 \\
\hline Animal & Cage & Group & Neutrophils-06 & Animal & Cage & Group & Neutrophils-06 \\
\hline 22 & 11 & NK33+ & 14.5 & 160 & 80 & NK11+ & 45.0 \\
\hline 9 & 5 & NK33+ & 15.5 & 196 & 98 & NK11+ & 43.0 \\
\hline 156 & 78 & NK11+ & 16.0 & 228 & 114 & NK33- & 40.0 \\
\hline 80 & 40 & NK11- & 16.5 & 269 & 135 & NK33- & 40.0 \\
\hline 217 & 109 & NK33- & 16.5 & 222 & 111 & NK33- & 39.5 \\
\hline Animal & Cage & Group & Neutrophils-12 & Animal & Cage & Group & Neutrophils-12 \\
\hline 35 & 18 & NK33+ & 19.5 & 153 & 77 & NK11+ & 81.0 \\
\hline
\end{tabular}


Statistical analysis of differential white blood cell counts for G-TwYST studies A and C

\begin{tabular}{|r|r|r|r|r|r|r|r|}
\hline 36 & 18 & NK33+ & 22.0 & 202 & 101 & NK11+ & 55.0 \\
\hline 80 & 40 & NK11- & 24.0 & 81 & 41 & NK11- & 54.0 \\
\hline 232 & 116 & NK33- & 24.0 & 195 & 98 & NK11+ & 54.0 \\
\hline 280 & 140 & NK33- & 24.0 & 221 & 111 & NK33- & 53.0 \\
\hline Animal & Cage & Group & Neutrophils-24 & Animal & Cage & Group & Neutrophils-24 \\
\hline 174 & 87 & NK11+ & 12.0 & 130 & 65 & NK11- & 91.0 \\
\hline 305 & 153 & Control & 15.0 & 36 & 18 & NK33+ & 82.5 \\
\hline 22 & 11 & NK33+ & 17.5 & 162 & 81 & NK11+ & 81.0 \\
\hline 117 & 59 & NK11- & 25.5 & 327 & 164 & Control & 79.5 \\
\hline 165 & 83 & NK11+ & 27.0 & 123 & 62 & NK11- & 78.0 \\
\hline
\end{tabular}

\begin{tabular}{|c|c|c|c|c|c|c|c|}
\hline \multicolumn{4}{|c|}{ Smallest five Monocytes Males } & \multicolumn{4}{|c|}{ Largest five Monocytes Males } \\
\hline Animal & Cage & Group & Monocytes-03 & Animal & Cage & Group & Monocytes-03 \\
\hline 17 & 9 & NK33+ & 0.5 & 73 & 37 & NK11- & 4.5 \\
\hline 27 & 14 & NK33+ & 0.5 & 74 & 37 & NK11- & 4.0 \\
\hline 80 & 40 & NK11- & 0.5 & 89 & 45 & NK11- & 4.0 \\
\hline 122 & 61 & NK11- & 0.5 & 3 & 2 & NK33+ & 3.5 \\
\hline 149 & 75 & NK11+ & 0.5 & 6 & 3 & NK33+ & 3.5 \\
\hline Animal & Cage & Group & Monocytes-06 & Animal & Cage & Group & Monocytes-06 \\
\hline 65 & 33 & NK33+ & 0.0 & 224 & 112 & NK33- & 3.0 \\
\hline 71 & 36 & NK11- & 0.0 & 297 & 149 & Control & 2.5 \\
\hline 89 & 45 & NK11- & 0.0 & 10 & 5 & NK33+ & 2.0 \\
\hline 135 & 68 & NK11- & 0.0 & 28 & 14 & NK33+ & 2.0 \\
\hline 136 & 68 & NK11- & 0.0 & 148 & 74 & NK11+ & 2.0 \\
\hline Animal & Cage & Group & Monocytes-12 & Animal & Cage & Group & Monocytes-12 \\
\hline 3 & 2 & NK33+ & 0.0 & 8 & 4 & NK33+ & 3.0 \\
\hline 15 & 8 & NK33+ & 0.0 & 265 & 133 & NK33- & 3.0 \\
\hline 16 & 8 & NK33+ & 0.0 & 81 & 41 & NK11- & 2.0 \\
\hline 17 & 9 & NK33+ & 0.0 & 196 & 98 & NK11+ & 2.0 \\
\hline 18 & 9 & NK33+ & 0.0 & 7 & 4 & NK33+ & 1.5 \\
\hline Animal & Cage & Group & Monocytes-24 & Animal & Cage & Group & Monocytes-24 \\
\hline 36 & 18 & NK33+ & 0.0 & 334 & 167 & Control & 11.0 \\
\hline 45 & 23 & NK33+ & 0.0 & 317 & 159 & Control & 10.5 \\
\hline 106 & 53 & NK11- & 0.0 & 139 & 70 & NK11- & 10.0 \\
\hline 122 & 61 & NK11- & 0.0 & 345 & 173 & Control & 10.0 \\
\hline 162 & 81 & NK11+ & 0.0 & 59 & 30 & NK33+ & 8.5 \\
\hline
\end{tabular}

\begin{tabular}{|r|r|r|r|r|r|r|r|}
\hline \multicolumn{6}{|c|}{ Smallest five Eosinophils Males } & \multicolumn{3}{|c|}{ Largest five Eosinophils Males } \\
\hline Animal & Cage & Group & Eosinophils-03 & Animal & Cage & Group & Eosinophils-03 \\
\hline 17 & 9 & NK33+ & 0.0 & 79 & 40 & NK11- & 7.0 \\
\hline 27 & 14 & NK33+ & 0.0 & 15 & 8 & NK33+ & 6.0 \\
\hline 39 & 20 & NK33+ & 0.0 & 290 & 145 & Control & 5.5 \\
\hline 98 & 49 & NK11- & 0.0 & 55 & 28 & NK33+ & 5.0 \\
\hline 129 & 65 & NK11- & 0.0 & 175 & 88 & NK11+ & 5.0 \\
\hline Animal & Cage & Group & Eosinophils-06 & Animal & Cage & Group & Eosinophils-06 \\
\hline 75 & 38 & NK11- & 0.0 & 159 & 80 & NK11+ & 10.5 \\
\hline 141 & 71 & NK11+ & 0.0 & 230 & 115 & NK33- & 8.0 \\
\hline 155 & 78 & NK11+ & 0.0 & 245 & 123 & NK33- & 6.5 \\
\hline 210 & 105 & NK11+ & 0.0 & 167 & 84 & NK11+ & 6.0 \\
\hline 249 & 125 & NK33- & 0.0 & 292 & 146 & Control & 6.0 \\
\hline Animal & Cage & Group & Eosinophils-12 & Animal & Cage & Group & Eosinophils-12 \\
\hline 12 & 6 & NK33+ & 0.0 & 89 & 45 & NK11- & 6.5 \\
\hline 15 & 8 & NK33+ & 0.0 & 84 & 42 & NK11- & 6.0 \\
\hline
\end{tabular}


Statistical analysis of differential white blood cell counts for G-TwYST studies A and C

\begin{tabular}{|r|r|r|r|r|r|r|r|}
\hline 21 & 11 & NK33+ & 0.0 & 153 & 77 & NK11+ & 6.0 \\
\hline 55 & 28 & NK33+ & 0.0 & 13 & 7 & NK33+ & 5.5 \\
\hline 77 & 39 & NK11- & 0.0 & 152 & 76 & NK11+ & 5.0 \\
\hline Animal & Cage & Group & Eosinophils-24 & Animal & Cage & Group & Eosinophils-24 \\
\hline 22 & 11 & NK33+ & 0.0 & 105 & 53 & NK11- & 5.0 \\
\hline 25 & 13 & NK33+ & 0.0 & 190 & 95 & NK11+ & 5.0 \\
\hline 48 & 24 & NK33+ & 0.0 & 201 & 101 & NK11+ & 5.0 \\
\hline 61 & 31 & NK33+ & 0.0 & 232 & 116 & NK33- & 4.0 \\
\hline 67 & 34 & NK33+ & 0.0 & 243 & 122 & NK33- & 4.0 \\
\hline
\end{tabular}

Table 4 The five smallest and five largest observed diffWBC percentages for every response variable observed in study A for Females. Outliers are listed in yellow.

\begin{tabular}{|r|r|r|r|r|r|r|r|}
\hline \multicolumn{4}{|c|}{ Smallest five Lymphocytes Females } & \multicolumn{3}{|c|}{ Largest five Lymphocytes Females } \\
\hline Animal & Cage & Group & Lymphocytes-03 & Animal & Cage & Group & Lymphocytes-03 \\
\hline 616 & 558 & NK11- & 20.0 & 792 & 646 & Control & 87.0 \\
\hline 729 & 615 & NK33- & 51.0 & 566 & 533 & NK33+ & 86.5 \\
\hline 571 & 536 & NK11- & 51.5 & 724 & 612 & NK33- & 86.0 \\
\hline 836 & 668 & Control & 52.0 & 765 & 633 & NK33- & 86.0 \\
\hline 641 & 571 & NK11+ & 56.5 & 845 & 673 & Control & 86.0 \\
\hline Animal & Cage & Group & Lymphocytes-06 & Animal & Cage & Group & Lymphocytes-06 \\
\hline 616 & 558 & NK11- & 24.0 & 513 & 507 & NK33+ & 83.0 \\
\hline 782 & 641 & Control & 52.0 & 795 & 648 & Control & 80.0 \\
\hline 629 & 565 & NK11- & 55.5 & 514 & 507 & NK33+ & 78.5 \\
\hline 640 & 570 & NK11- & 55.5 & 516 & 508 & NK33+ & 78.5 \\
\hline 709 & 605 & NK11+ & 57.0 & 586 & 543 & NK11- & 78.5 \\
\hline Animal & Cage & Group & Lymphocytes-12 & Animal & Cage & Group & Lymphocytes-12 \\
\hline 585 & 543 & NK11- & 33.0 & 711 & 606 & NK33- & 91.5 \\
\hline 727 & 614 & NK33- & 41.5 & 641 & 571 & NK11+ & 88.5 \\
\hline 815 & 658 & Control & 43.0 & 724 & 612 & NK33- & 86.5 \\
\hline 643 & 572 & NK11+ & 46.0 & 781 & 641 & Control & 85.5 \\
\hline 825 & 663 & Control & 46.0 & 788 & 644 & Control & 85.0 \\
\hline Animal & Cage & Group & Lymphocytes-24 & Animal & Cage & Group & Lymphocytes-24 \\
\hline 592 & 546 & NK11- & 9.0 & 702 & 601 & NK11+ & 80.0 \\
\hline 671 & 586 & NK11+ & 11.0 & 568 & 534 & NK33+ & 79.5 \\
\hline 521 & 511 & NK33+ & 17.0 & 567 & 534 & NK33+ & 79.0 \\
\hline 664 & 582 & NK11+ & 21.0 & 602 & 551 & NK11- & 79.0 \\
\hline 831 & 666 & Control & 22.0 & 690 & 595 & NK11+ & 77.5 \\
\hline
\end{tabular}

\begin{tabular}{|r|r|r|r|r|r|r|r|}
\hline \multicolumn{4}{|c|}{ Smallest five Neutrophils Females } & \multicolumn{4}{|c|}{ Largest five Neutrophils Females } \\
\hline Animal & Cage & Group & Neutrophils-03 & Animal & Cage & Group & Neutrophils-03 \\
\hline 566 & 533 & NK33+ & 10.0 & 616 & 558 & NK11- & 73.5 \\
\hline 792 & 646 & Control & 11.0 & 729 & 615 & NK33- & 47.5 \\
\hline 845 & 673 & Control & 12.0 & 571 & 536 & NK11- & 43.5 \\
\hline 802 & 651 & Control & 12.5 & 680 & 590 & NK11+ & 40.0 \\
\hline 724 & 612 & NK33- & 13.0 & 575 & 538 & NK11- & 39.5 \\
\hline Animal & Cage & Group & Neutrophils-06 & Animal & Cage & Group & Neutrophils-06 \\
\hline 513 & 507 & NK33+ & 16.5 & 616 & 558 & NK11- & 69.5 \\
\hline 795 & 648 & Control & 17.0 & 782 & 641 & Control & 45.0 \\
\hline 651 & 576 & NK11+ & 19.0 & 727 & 614 & NK33- & 41.0 \\
\hline 808 & 654 & Control & 19.0 & 629 & 565 & NK11- & 40.5 \\
\hline 626 & 563 & NK11- & 19.5 & 709 & 605 & NK11+ & 40.0 \\
\hline
\end{tabular}


Statistical analysis of differential white blood cell counts for G-TwYST studies A and C

\begin{tabular}{|r|r|r|r|r|r|r|r|}
\hline Animal & Cage & Group & Neutrophils-12 & Animal & Cage & Group & Neutrophils-12 \\
\hline 711 & 606 & NK33- & 3.5 & 585 & 543 & NK11- & 63.5 \\
\hline 724 & 612 & NK33- & 10.5 & 727 & 614 & NK33- & 56.5 \\
\hline 712 & 606 & NK33- & 11.0 & 643 & 572 & NK11+ & 52.0 \\
\hline 641 & 571 & NK11+ & 11.5 & 606 & 553 & NK11- & 50.5 \\
\hline 781 & 641 & Control & 12.5 & 730 & 615 & NK33- & 50.5 \\
\hline Animal & Cage & Group & Neutrophils-24 & Animal & Cage & Group & Neutrophils-24 \\
\hline 568 & 534 & NK33+ & 17.0 & 592 & 546 & NK11- & 88.0 \\
\hline 602 & 551 & NK11- & 18.0 & 521 & 511 & NK33+ & 81.5 \\
\hline 567 & 534 & NK33+ & 18.5 & 671 & 586 & NK11+ & 77.0 \\
\hline 702 & 601 & NK11+ & 19.0 & 664 & 582 & NK11+ & 75.0 \\
\hline 690 & 595 & NK11+ & 20.0 & 771 & 636 & NK33- & 75.0 \\
\hline
\end{tabular}

\begin{tabular}{|c|c|c|c|c|c|c|c|}
\hline \multicolumn{4}{|c|}{ Smallest five Monocytes Females } & \multicolumn{4}{|c|}{ Largest five Monocytes Females } \\
\hline Animal & Cage & Group & Monocytes-03 & Animal & Cage & Group & Monocytes-03 \\
\hline 574 & 537 & NK11- & 0.0 & 503 & 502 & NK33+ & 3.5 \\
\hline 514 & 507 & NK33+ & 0.5 & 540 & 520 & NK33+ & 3.5 \\
\hline 573 & 537 & NK11- & 0.5 & 577 & 539 & NK11- & 3.5 \\
\hline 616 & 558 & NK11- & 0.5 & 578 & 539 & NK11- & 3.5 \\
\hline 653 & 577 & NK11+ & 0.5 & 642 & 571 & NK11+ & 3.5 \\
\hline Animal & Cage & Group & Monocytes-06 & Animal & Cage & Group & Monocytes-06 \\
\hline 508 & 504 & NK33+ & 0.0 & 616 & 558 & NK11- & 3.5 \\
\hline 513 & 507 & NK33+ & 0.0 & 755 & 628 & NK33- & 3.0 \\
\hline 516 & 508 & NK33+ & 0.0 & 714 & 607 & NK33- & 2.5 \\
\hline 519 & 510 & NK33+ & 0.0 & 784 & 642 & Control & 2.5 \\
\hline 528 & 514 & NK33+ & 0.0 & 578 & 539 & NK11- & 2.0 \\
\hline Animal & Cage & Group & Monocytes-12 & Animal & Cage & Group & Monocytes-12 \\
\hline 507 & 504 & NK33+ & 0.0 & 799 & 650 & Control & 7.0 \\
\hline 508 & 504 & NK33+ & 0.0 & 552 & 526 & NK33+ & 5.0 \\
\hline 509 & 505 & NK33+ & 0.0 & 659 & 580 & NK11+ & 4.5 \\
\hline 510 & 505 & NK33+ & 0.0 & 815 & 658 & Control & 4.5 \\
\hline 512 & 506 & NK33+ & 0.0 & 755 & 628 & NK33- & 4.0 \\
\hline Animal & Cage & Group & Monocytes-24 & Animal & Cage & Group & Monocytes-24 \\
\hline 553 & 527 & NK33+ & 0.0 & 671 & 586 & NK11+ & 10.5 \\
\hline 630 & 565 & NK11- & 0.0 & 831 & 666 & Control & 8.5 \\
\hline 631 & 566 & NK11- & 0.0 & 540 & 520 & NK33+ & 8.0 \\
\hline 688 & 594 & NK11+ & 0.0 & 618 & 559 & NK11- & 7.5 \\
\hline 698 & 599 & NK11+ & 0.0 & 823 & 662 & Control & 7.0 \\
\hline
\end{tabular}

\begin{tabular}{|r|r|r|r|r|r|r|r|}
\hline \multicolumn{4}{|c|}{ Smallest five Eosinophils Females } & \multicolumn{4}{|c|}{ Largest five Eosinophils Females } \\
\hline Animal & Cage & Group & Eosinophils-03 & Animal & Cage & Group & Eosinophils-03 \\
\hline 540 & 520 & NK33+ & 0.0 & 826 & 663 & Control & 8.5 \\
\hline 569 & 535 & NK33+ & 0.0 & 836 & 668 & Control & 6.5 \\
\hline 570 & 535 & NK33+ & 0.0 & 555 & 528 & NK33+ & 6.0 \\
\hline 573 & 537 & NK11- & 0.0 & 616 & 558 & NK11- & 6.0 \\
\hline 576 & 538 & NK11- & 0.0 & 517 & 509 & NK33+ & 5.0 \\
\hline Animal & Cage & Group & Eosinophils-06 & Animal & Cage & Group & Eosinophils-06 \\
\hline 508 & 504 & NK33+ & 0.0 & 577 & 539 & NK11- & 6.0 \\
\hline 622 & 561 & NK11- & 0.0 & 517 & 509 & NK33+ & 5.0 \\
\hline 769 & 635 & NK33- & 0.0 & 539 & 520 & NK33+ & 5.0 \\
\hline 507 & 504 & NK33+ & 0.5 & 762 & 631 & NK33- & 5.0 \\
\hline 509 & 505 & NK33+ & 0.5 & 796 & 648 & Control & 5.0 \\
\hline
\end{tabular}


Statistical analysis of differential white blood cell counts for G-TwYST studies A and C

\begin{tabular}{|r|r|r|r|r|r|r|r|}
\hline Animal & Cage & Group & Eosinophils-12 & Animal & Cage & Group & Eosinophils-12 \\
\hline 522 & 511 & NK33+ & 0.0 & 580 & 540 & NK11- & 10.5 \\
\hline 612 & 556 & NK11- & 0.0 & 598 & 549 & NK11- & 6.0 \\
\hline 629 & 565 & NK11- & 0.0 & 718 & 609 & NK33- & 5.0 \\
\hline 641 & 571 & NK11+ & 0.0 & 552 & 526 & NK33+ & 4.5 \\
\hline 643 & 572 & NK11+ & 0.0 & 711 & 606 & NK33- & 4.5 \\
\hline Animal & Cage & Group & Eosinophils-24 & Animal & Cage & Group & Eosinophils-24 \\
\hline 533 & 517 & NK33+ & 0.0 & 527 & 514 & NK33+ & 4.0 \\
\hline 534 & 517 & NK33+ & 0.0 & 619 & 560 & NK11- & 4.0 \\
\hline 535 & 518 & NK33+ & 0.0 & 734 & 617 & NK33- & 4.0 \\
\hline 540 & 520 & NK33+ & 0.0 & 748 & 624 & NK33- & 4.0 \\
\hline 541 & 521 & NK33+ & 0.0 & 756 & 628 & NK33- & 4.0 \\
\hline
\end{tabular}

The cage means, after a log transformation, for each observed variable were statistically analysed by means of an analysis of variance using the model "Block + Group" according to the randomized block design. To circumvent taking the logarithm of zero, 0.5 was added to each Monocytes and Eosinophils observation before taking logs. Grubbs' outlier test at the $1 \%$ level was sequentially applied to the residuals to detect outliers. This resulted in the outliers listed below, and these were presented to the G-TwYST coordinator who confirmed the proposed outlier classifications:

1. Males, Lymphocytes-03, cage 133 , group NK33- (p-value 0.000$)$. This cage houses the two animals 266 and 265 with the two smallest Lymphocytes values (38.0 and 45.5, Table 3). These two values are considered to be outliers.

2. Males, Lymphocytes-12, cage 77 , group NK11+ (p-values 0.000 ). This cage houses animal 153 with by far the smallest Lymphocyte value $(12.0$, Table 3$)$. This value is considered to be an outlier.

3. Males, Lymphocytes-24, cage 65 , group NK11- (p-values 0.003 ). This cage houses animal 130 with by far the smallest Lymphocyte value (1.0, Table 3$)$. This value is considered to be an outlier.

4. Females, Lymphocytes-03, cage 615 , group NK33- (p-values 0.003 ); found after removing the value for animal 616 . Cage 615 houses animals 729 and 730 with values 51.0 and 62.5 , of which 51.0 is the second smallest (Table 4). These values are not considered to be extreme and are therefore not seen as outliers.

5. Females, Lymphocytes-24, cage 586 , group NK11+ ( $p$-values 0.005$)$. The cage mean for this cage is based on a single animal 671 with the second smallest value (11.0, Table 4). This value is not considered to be extreme and is therefore not seen as an outlier.

6. Females, Neutrophils-12, cage 606, group NK33- (p-values 0.000 ). This cage houses animal 711 with by far the smallest value (3.5, Table 4$)$. This value is considered to be an outlier.

7. Females, Monocytes-03, cage 537, group NK11- ( $p$-values 0.004$)$. This cage houses animal 574 and 573 with two of the smallest values ( 0.0 and 0.5 , Table 4 ). These values are not considered to be extreme and are therefore not seen as outliers.

Plots of residuals versus fitted values, after removal of the outliers listed above, are given in Figure 1 and Figure 3, while normal probability plots are given in Figure 2 and Figure 4 . These residual plots are generally satisfactory implying that the ANOVA assumptions, homogeneity of variance and less importantly normality, are generally fulfilled. 

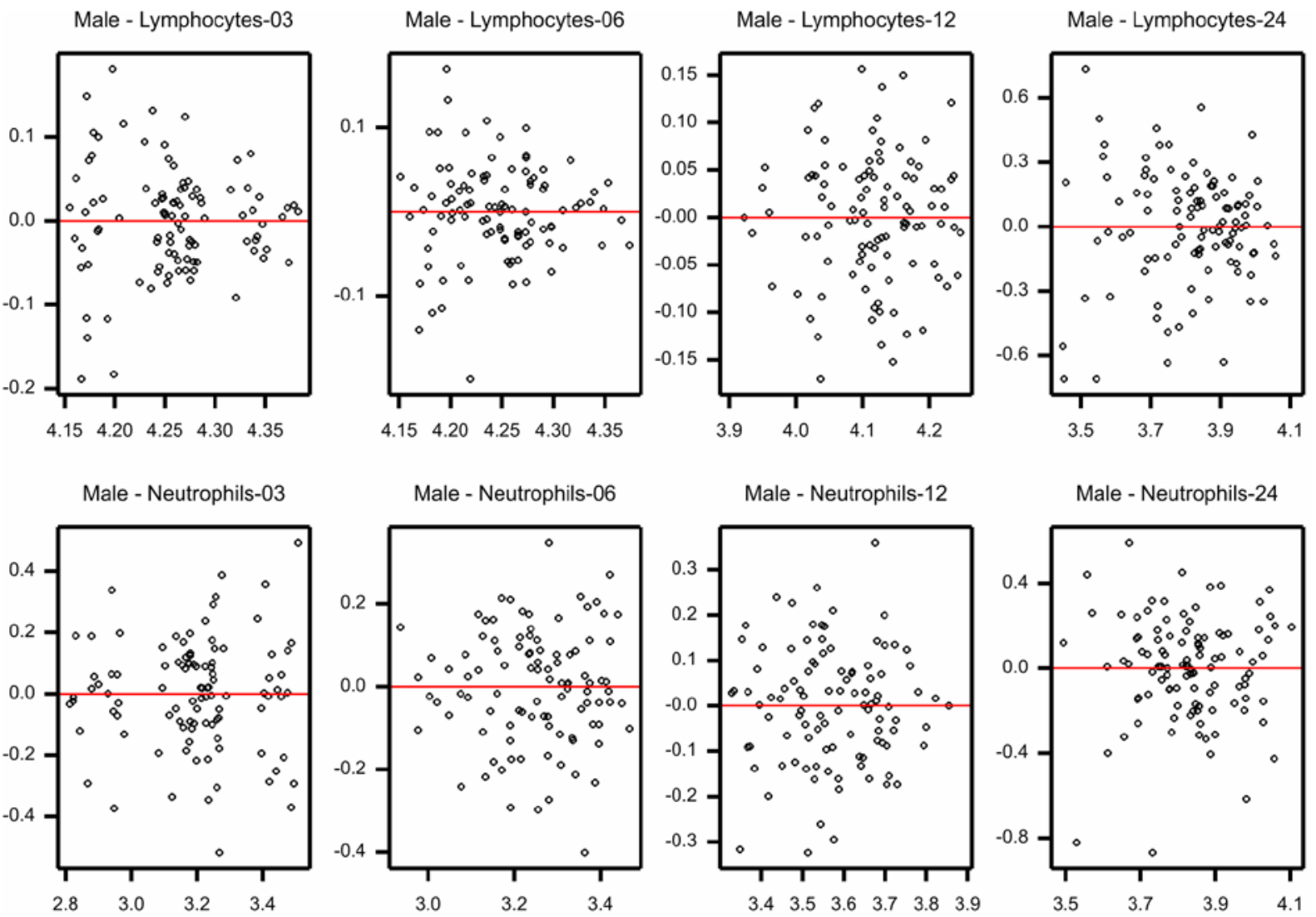

Male - Monocytes-03
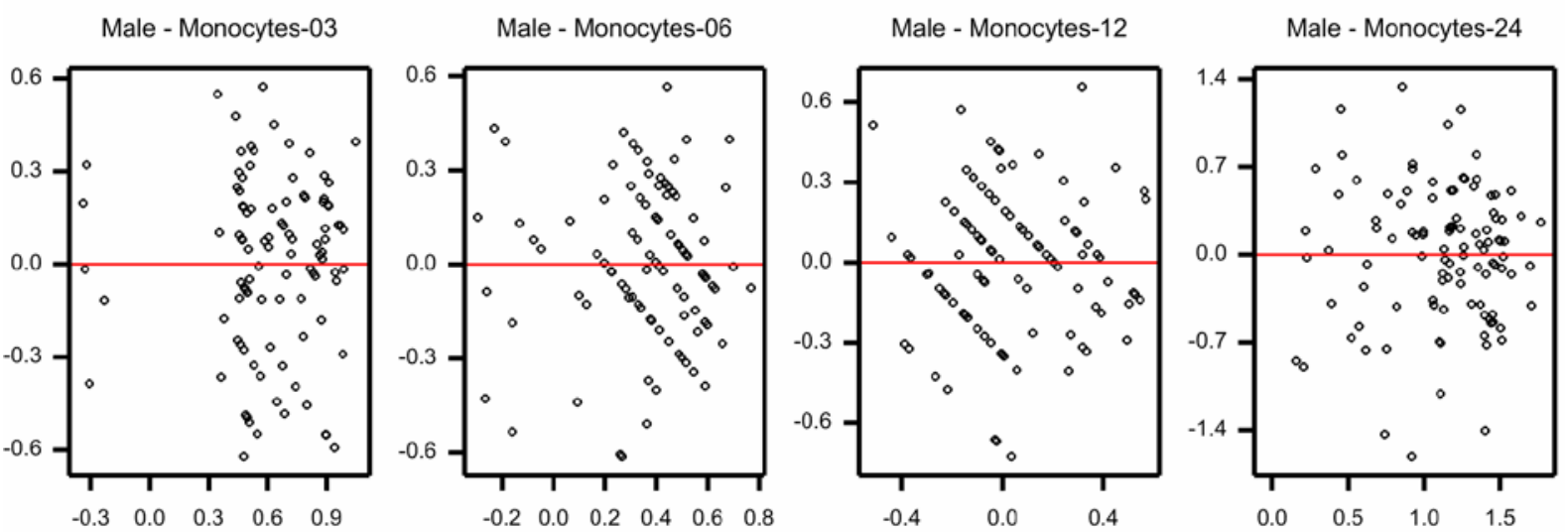

Male - Eosinophils-06

Male - Eosinophils-12

Male - Eosinophils-24
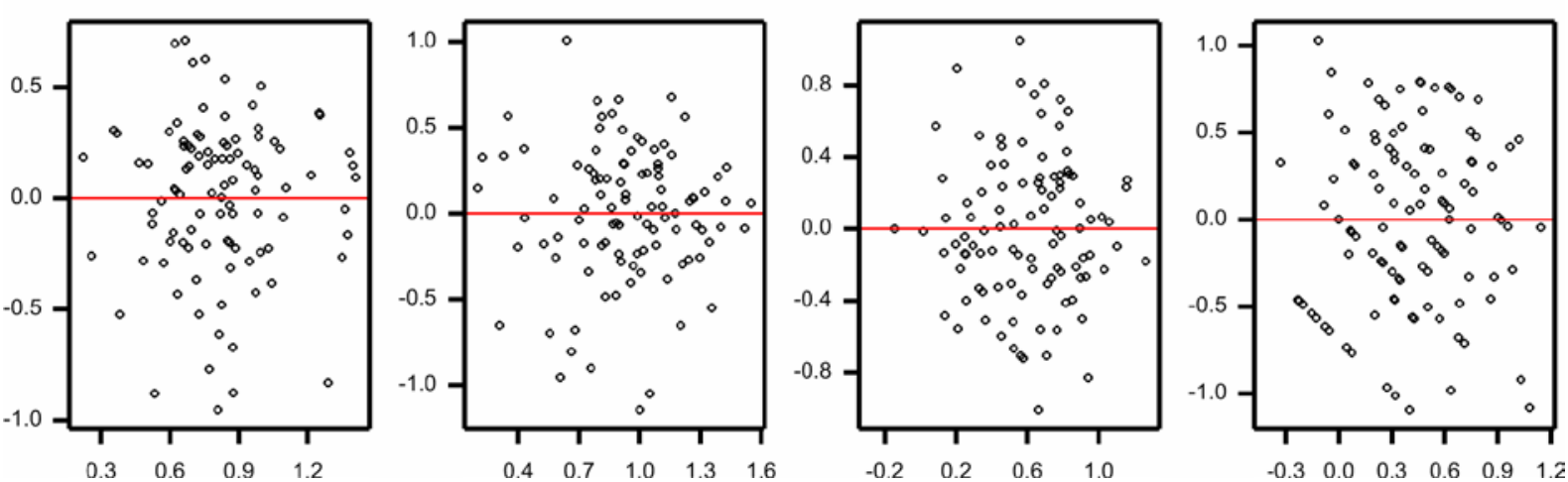

Figure 1 Residuals along the $y$-axis versus fitted values along the $x$-axis resulting from an analysis of variance for Study $A$ in Males on log transformed cage means. 

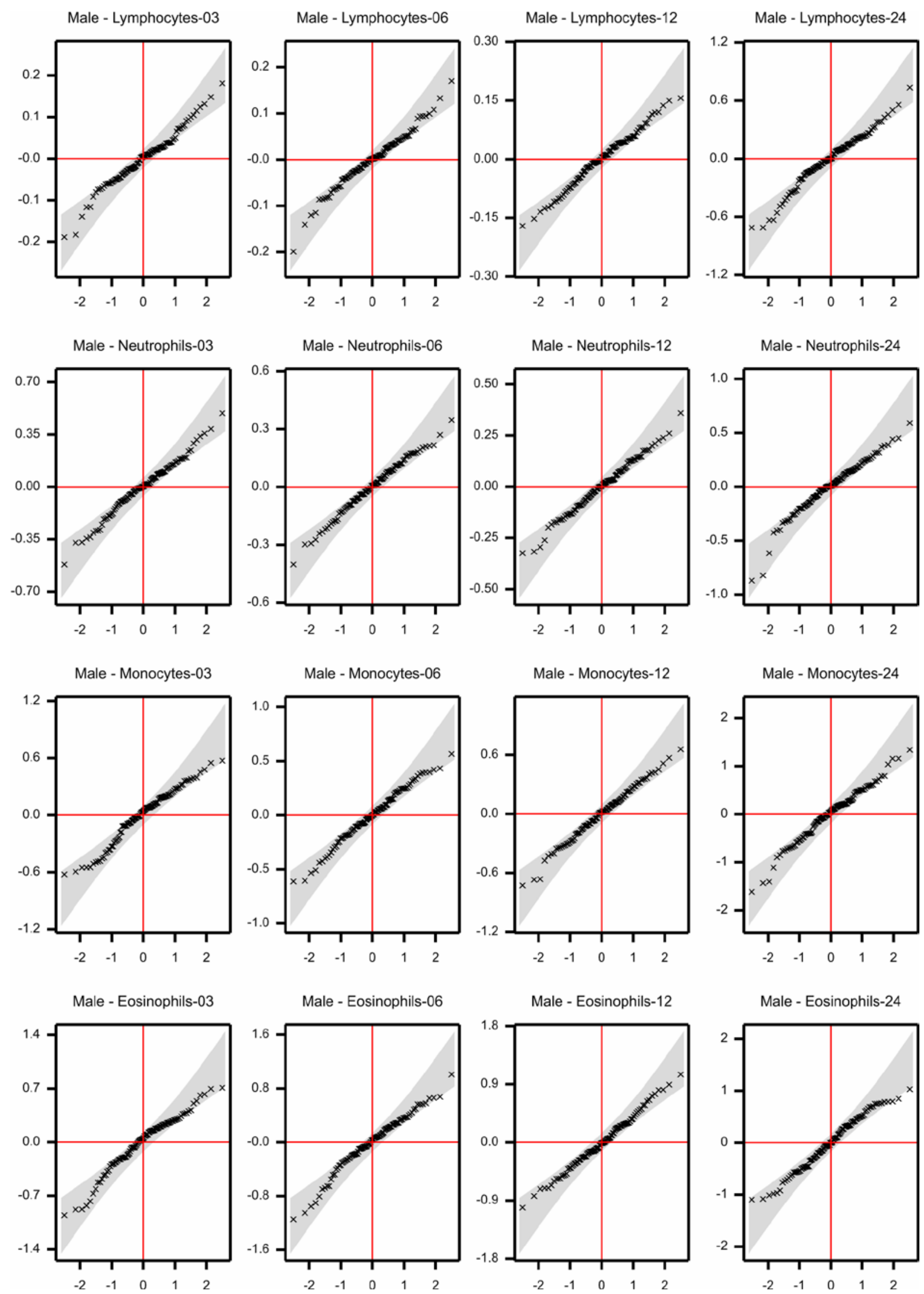

Figure 2 Normal probability plots of residuals resulting from an analysis of variance for Study A in Males on log transformed cage means. 

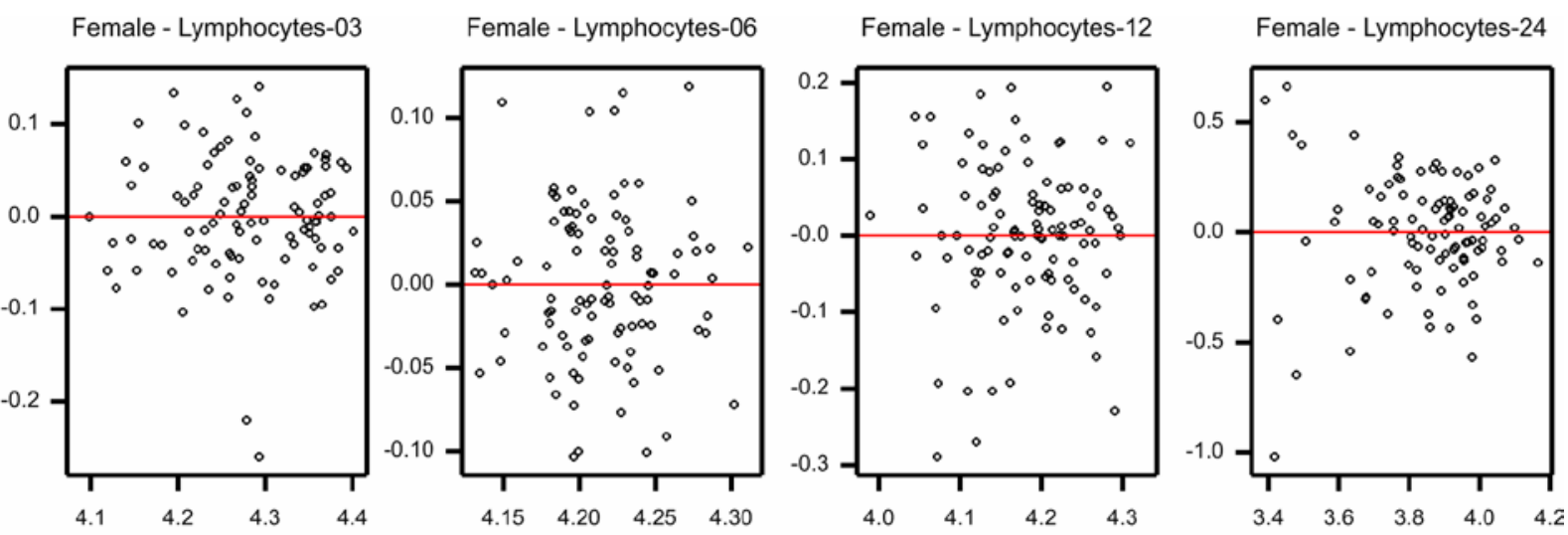

Female - Neutrophils-03

Female - Neutrophils-06

Female - Neutrophils-12

Female - Neutrophils-24
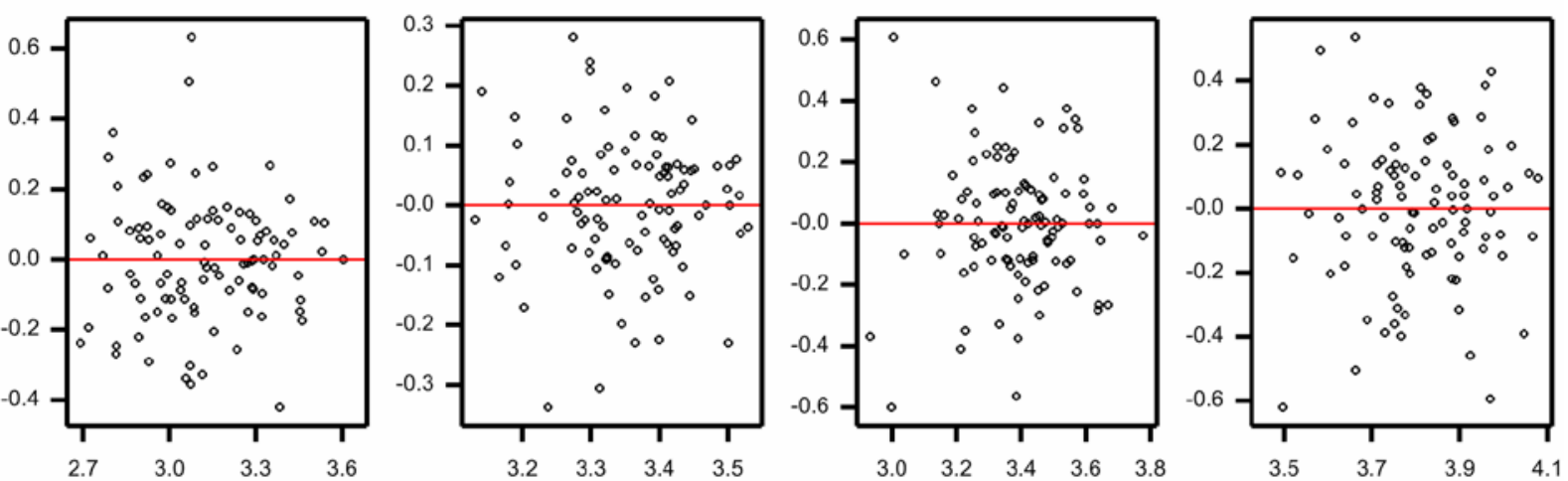

Female - Monocytes-03

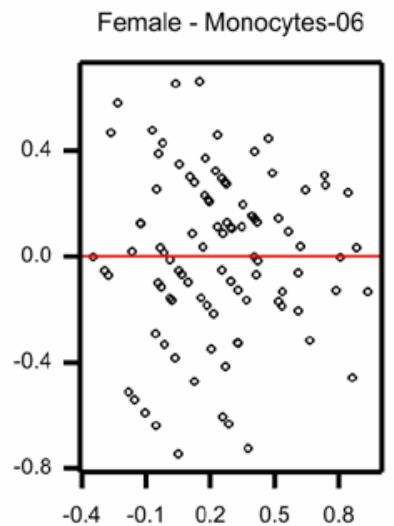

Female - Monocytes-12

Female - Monocytes-24
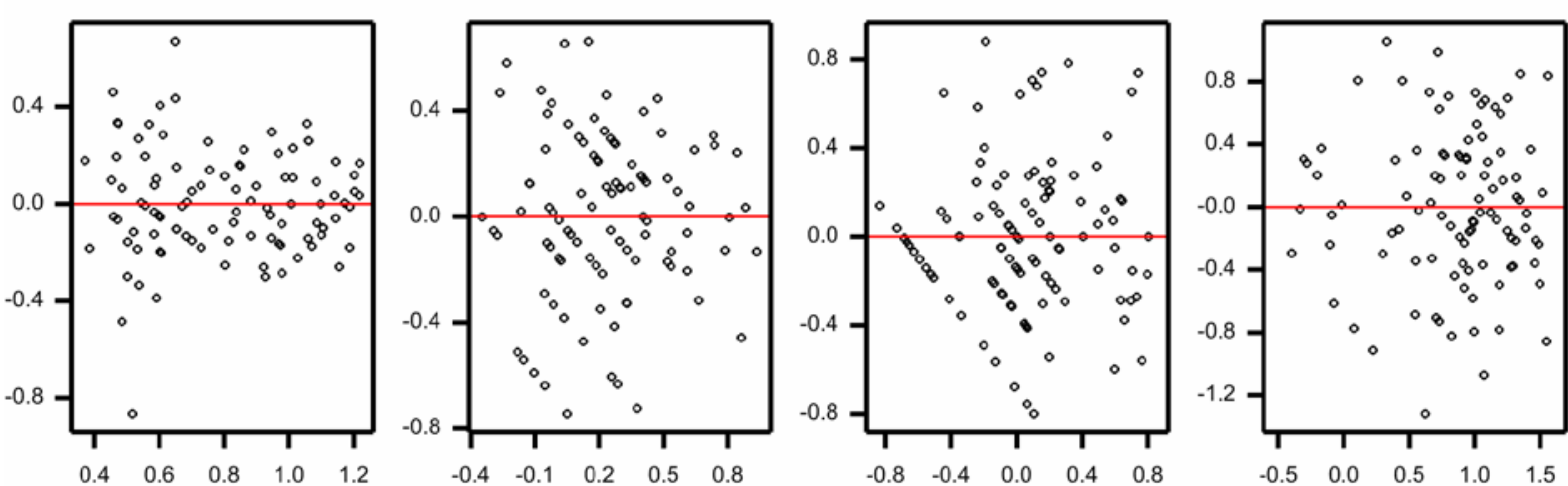

Female - Eosinophils-03

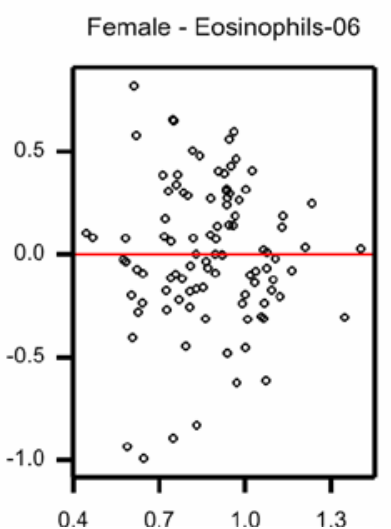

Female - Eosinophils-12

Female - Eosinophils-24
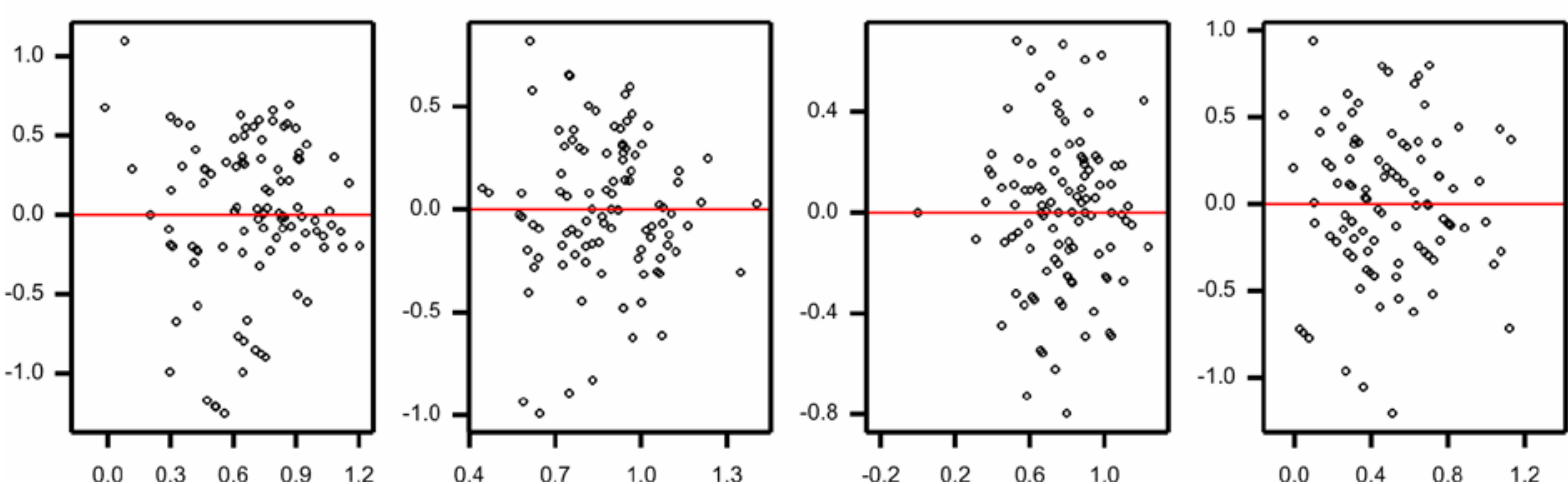

Figure 3 Residuals along the $y$-axis versus fitted values along the $x$-axis resulting from an analysis of variance for Study A in Females on log transformed cage means. 
Female - Lymphocytes-03

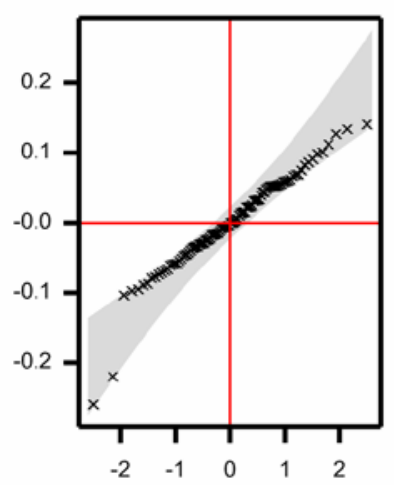

Female - Neutrophils-03

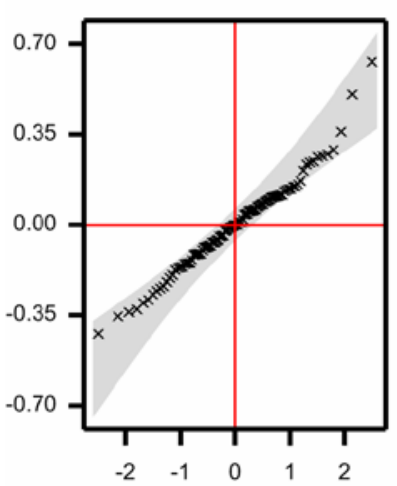

Female - Monocytes-03

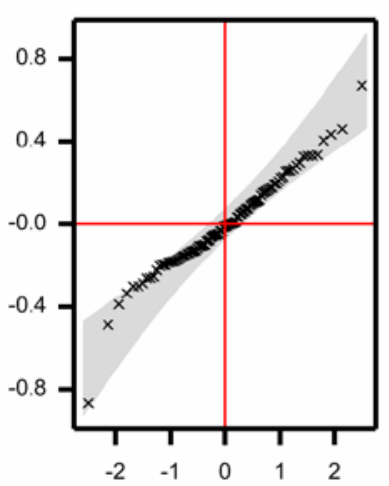

Female - Eosinophils-03

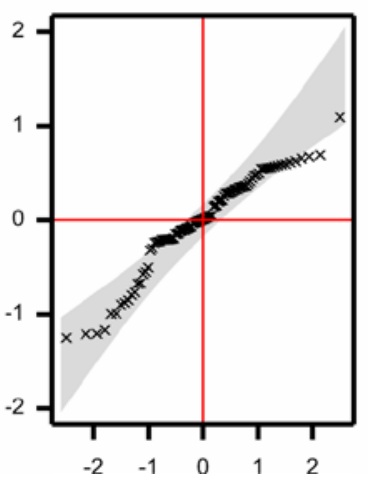

Female - Lymphocytes-06

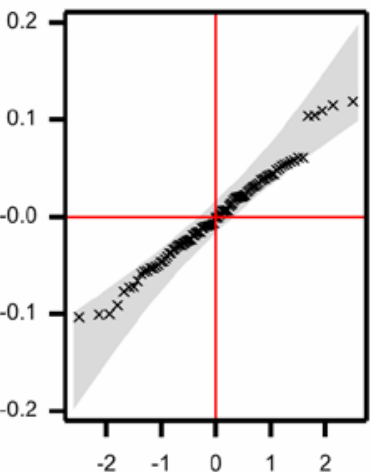

Female - Neutrophils-06

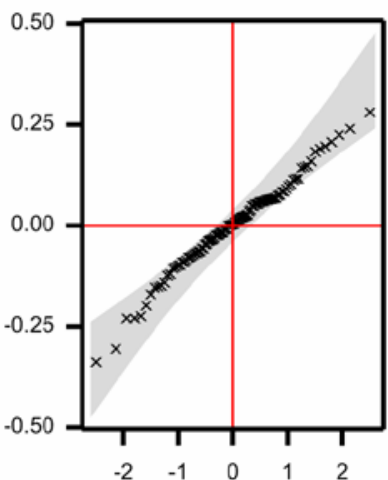

Female - Monocytes-06

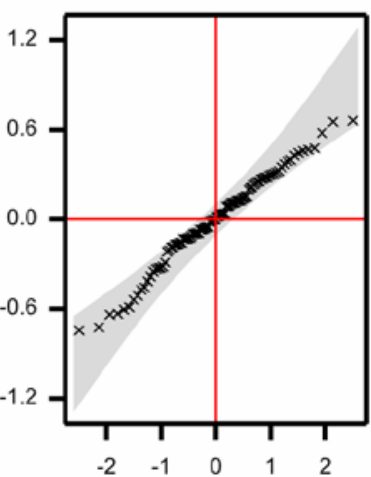

Female - Eosinophils-06

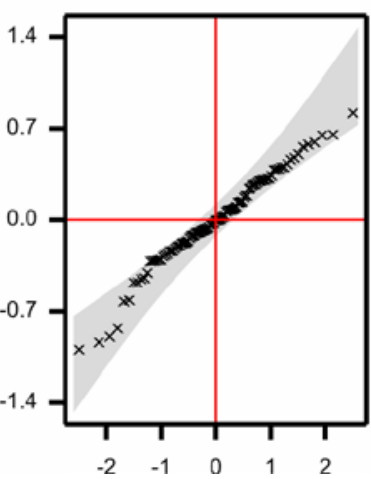

Female - Lymphocytes-12

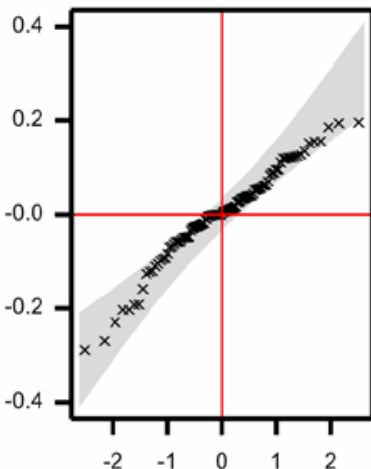

Female - Neutrophils-12

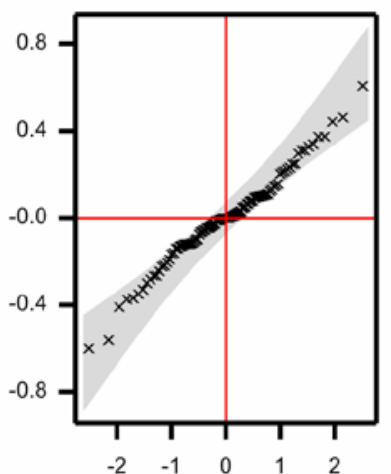

Female - Monocytes-12

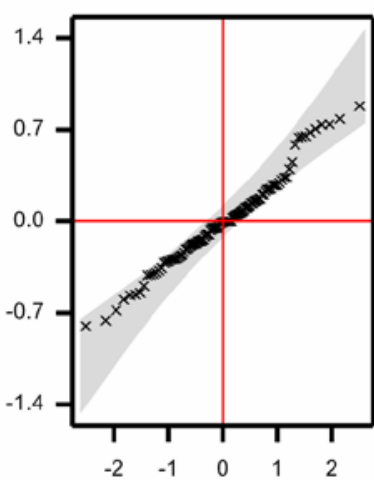

Female - Eosinophils-12

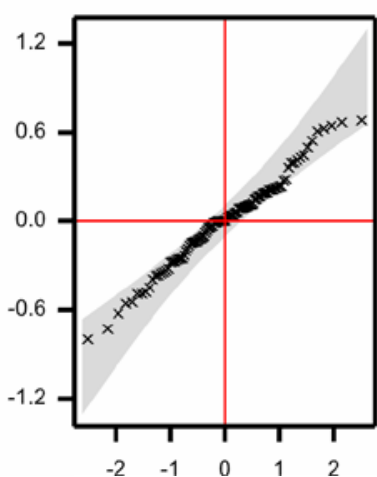

Female - Lymphocytes-24

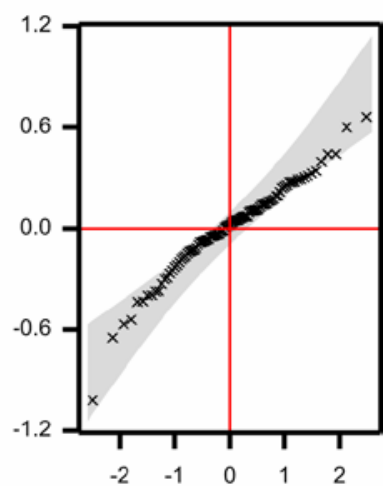

Female - Neutrophils-24

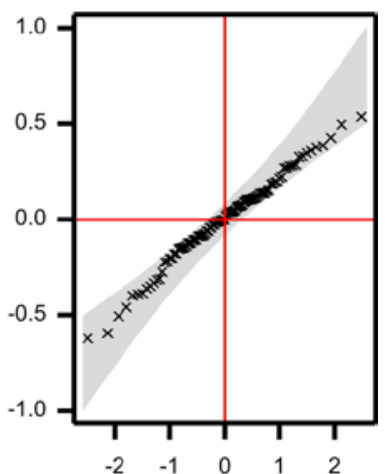

Female - Monocytes-24

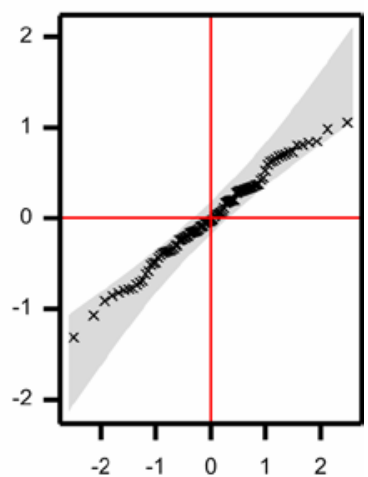

Female - Eosinophils-24

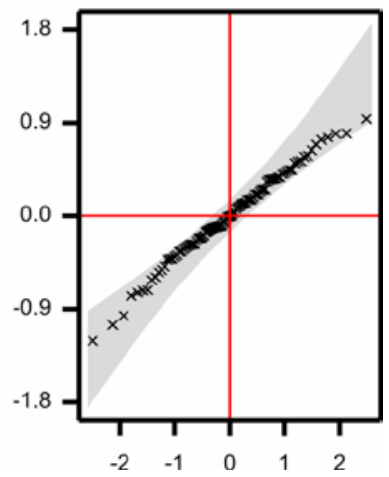

Figure 4 Normal probability plots of residuals resulting from an analysis of variance for Study A in Females on log transformed cage means. 


\subsection{Outliers and checking of ANOVA assumptions for study $C$}

Data on diffWBC are available for all 256 animals in Study C. Basophils were mainly zero, expect for a count of 1 for two male animals and for one female animal, and Basophils were therefore not statistically analysed. The five smallest and largest values are given in Table 5 and Table 6 .

Table 5 The five smallest and five largest observed diffWBC percentages for every response variable observed in study $C$ for Males. No outliers were identified.

\begin{tabular}{|r|r|r|r|r|r|r|r|}
\hline \multicolumn{4}{|c|}{ Five smallest Males } & \multicolumn{4}{|c|}{ Five largest Males } \\
\hline Animal & Cage & Group & Lymphocytes & Animal & Cage & Group & Lymphocytes \\
\hline 21 & 11 & NK50+ & 20.5 & 49 & 25 & NK11+/50 & 99.0 \\
\hline 118 & 59 & NK33+ & 29.5 & 1 & 1 & NK50- & 98.5 \\
\hline 102 & 51 & Con33 & 39.0 & 76 & 38 & NK11-/50 & 98.0 \\
\hline 109 & 55 & Con33 & 44.0 & 3 & 2 & NK50- & 97.5 \\
\hline 110 & 55 & Con33 & 44.0 & 68 & 34 & NK11-/50 & 97.5 \\
\hline Animal & Cage & Group & Neutrophils & Animal & Cage & Group & Neutrophils \\
\hline 1 & 1 & NK50- & 0.0 & 21 & 11 & NK50+ & 71.0 \\
\hline 2 & 1 & NK50- & 0.0 & 118 & 59 & NK33+ & 66.0 \\
\hline 68 & 34 & NK11-/50 & 0.0 & 102 & 51 & Con33 & 57.0 \\
\hline 76 & 38 & NK11-/50 & 0.0 & 109 & 55 & Con33 & 51.0 \\
\hline 3 & 2 & NK50- & 0.5 & 110 & 55 & Con33 & 50.5 \\
\hline Animal & Cage & Group & Monocytes & Animal & Cage & Group & Monocytes \\
\hline 1 & 1 & NK50- & 0.0 & 86 & 43 & NK33- & 8.5 \\
\hline 2 & 1 & NK50- & 0.0 & 79 & 40 & NK11-/50 & 6.5 \\
\hline 3 & 2 & NK50- & 0.0 & 90 & 45 & NK33- & 6.5 \\
\hline 4 & 2 & NK50- & 0.0 & 84 & 42 & NK33- & 6.0 \\
\hline 49 & 25 & NK11+/50 & 0.0 & 94 & 47 & NK33- & 5.5 \\
\hline Animal & Cage & Group & Eosinophils & Animal & Cage & Group & Eosinophils \\
\hline 6 & 3 & NK50- & 0.0 & 97 & 49 & Con33 & 11.0 \\
\hline 7 & 4 & NK50- & 0.0 & 21 & 11 & NK50+ & 6.5 \\
\hline 8 & 4 & NK50- & 0.0 & 88 & 44 & NK33- & 4.5 \\
\hline 19 & 10 & NK50+ & 0.0 & 2 & 1 & NK50- & 4.0 \\
\hline 29 & 15 & NK50+ & 0.0 & 22 & 11 & NK50+ & 4.0 \\
\hline
\end{tabular}

Table 6 The five smallest and five largest observed diffWBC percentages for every response variable observed in study $C$ for Females. No outliers were identified.

\begin{tabular}{|r|r|r|r|r|r|r|r|}
\hline \multicolumn{5}{|c|}{ Five smallest Females } & \multicolumn{4}{|c|}{ Five largest Females } \\
\hline Animal & Cage & Group & Lymphocytes & Animal & Cage & Group & Lymphocytes \\
\hline 246 & 90 & Con50 & 40.0 & 267 & 101 & NK11-/50 & 98.5 \\
\hline 256 & 95 & NK11+/50 & 46.0 & 317 & 126 & NK33+ & 98.0 \\
\hline 245 & 90 & Con50 & 52.0 & 221 & 78 & NK50+ & 97.5 \\
\hline 322 & 128 & NK33+ & 52.0 & 300 & 117 & Con33 & 97.0 \\
\hline 201 & 68 & NK50- & 55.5 & 299 & 117 & Con33 & 96.5 \\
\hline Animal & Cage & Group & Neutrophils & Animal & Cage & Group & Neutrophils \\
\hline 221 & 78 & NK50+ & 0.0 & 246 & 90 & Con50 & 56.5 \\
\hline 267 & 101 & NK11-/50 & 0.0 & 256 & 95 & NK11+/50 & 53.0 \\
\hline 317 & 126 & NK33+ & 1.0 & 245 & 90 & Con50 & 44.0 \\
\hline 300 & 117 & Con33 & 2.0 & 322 & 128 & NK33+ & 44.0 \\
\hline 308 & 121 & Con33 & 2.5 & 277 & 106 & NK11-/50 & 43.0 \\
\hline
\end{tabular}


Statistical analysis of differential white blood cell counts for G-TwYST studies A and C

\begin{tabular}{|r|r|r|r|r|r|r|r|}
\hline Animal & Cage & Group & Monocytes & Animal & Cage & Group & Monocytes \\
\hline 202 & 68 & NK50- & 0.0 & 287 & 111 & NK33- & 5.0 \\
\hline 214 & 74 & NK50- & 0.0 & 216 & 75 & NK50- & 3.5 \\
\hline 236 & 85 & Con50 & 0.0 & 223 & 79 & NK50+ & 3.5 \\
\hline 241 & 88 & Con50 & 0.0 & 246 & 90 & Con50 & 3.5 \\
\hline 243 & 89 & Con50 & 0.0 & 273 & 104 & NK11-/50 & 3.5 \\
\hline Animal & Cage & Group & Eosinophils & Animal & Cage & Group & Eosinophils \\
\hline 212 & 73 & NK50- & 0.0 & 229 & 82 & NK50+ & 4.5 \\
\hline 215 & 75 & NK50- & 0.0 & 201 & 68 & NK50- & 4.0 \\
\hline 236 & 85 & Con50 & 0.0 & 292 & 113 & NK33- & 4.0 \\
\hline 237 & 86 & Con50 & 0.0 & 295 & 115 & NK33- & 4.0 \\
\hline 246 & 90 & Con50 & 0.0 & 235 & 85 & Con50 & 3.5 \\
\hline
\end{tabular}

The four diffWBC variables were log transformed after which cage means were calculated. Note that Neutrophils, Monocytes and Eosinophils contain zero counts, and therefore for these variables 0.5 was added to each value before taking the logarithm. The log-transformed data were statistically analysed by means of an analysis of variance using the model "Block + Group", which is according to the randomized block design. Grubbs' outlier test at the $1 \%$ level was applied to the residuals to detect outliers. This resulted in a single outlier for Lymphocytes observed in females ( $p$-value for Grubbs' test equals 0.009 which is just significant at the $1 \%$ level). The outlier was observed for Cage 90 for feeding group Con50 with animals 245 and 246 which had two of the lowest values (40.0 and 52.0, Table 6). These values are not considered to be extreme and are therefore, after consultation of the G-TwYST coordinator, not seen as outliers.

Plots of residuals versus fitted values and normal probability plots are given in Figure 5 . These residual plots are generally satisfactory implying that the ANOVA assumptions, homogeneity of variance and less importantly normality, are generally fulfilled. Note that for Neutrophils there is some indication that smaller values are more variable than larger values. 
Statistical analysis of differential white blood cell counts for G-TwYST studies A and C
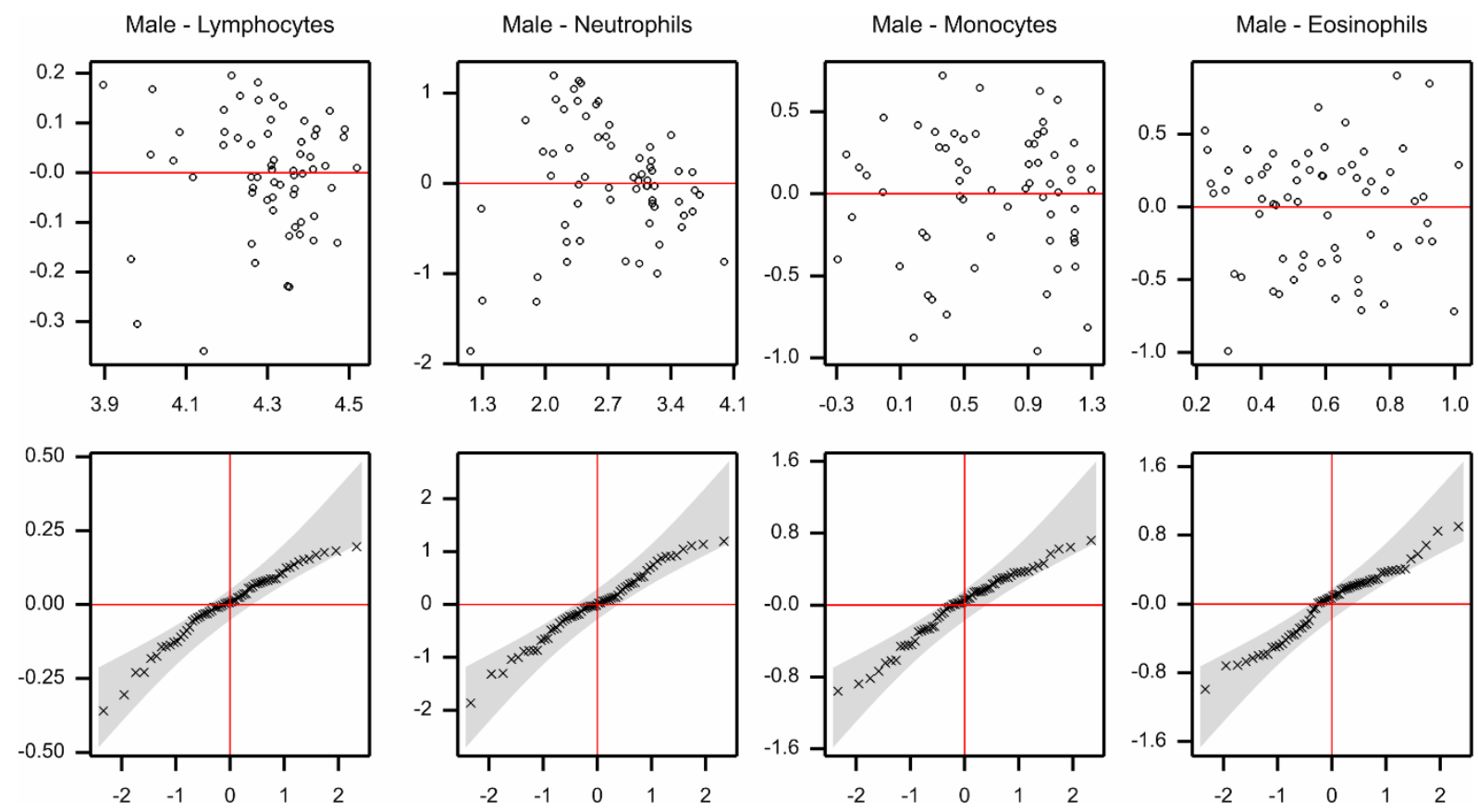

Female - Lymphocytes

Female - Neutrophils
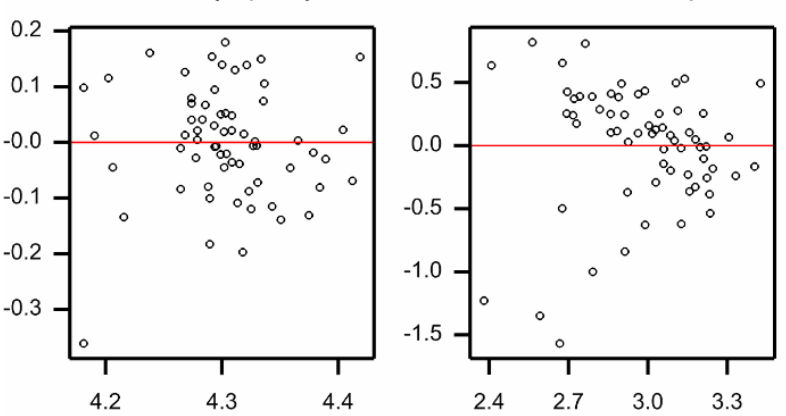

Female - Monocytes

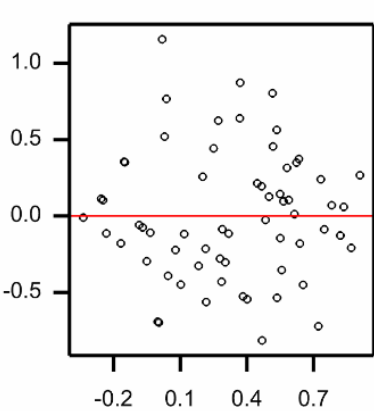

Female - Eosinophils
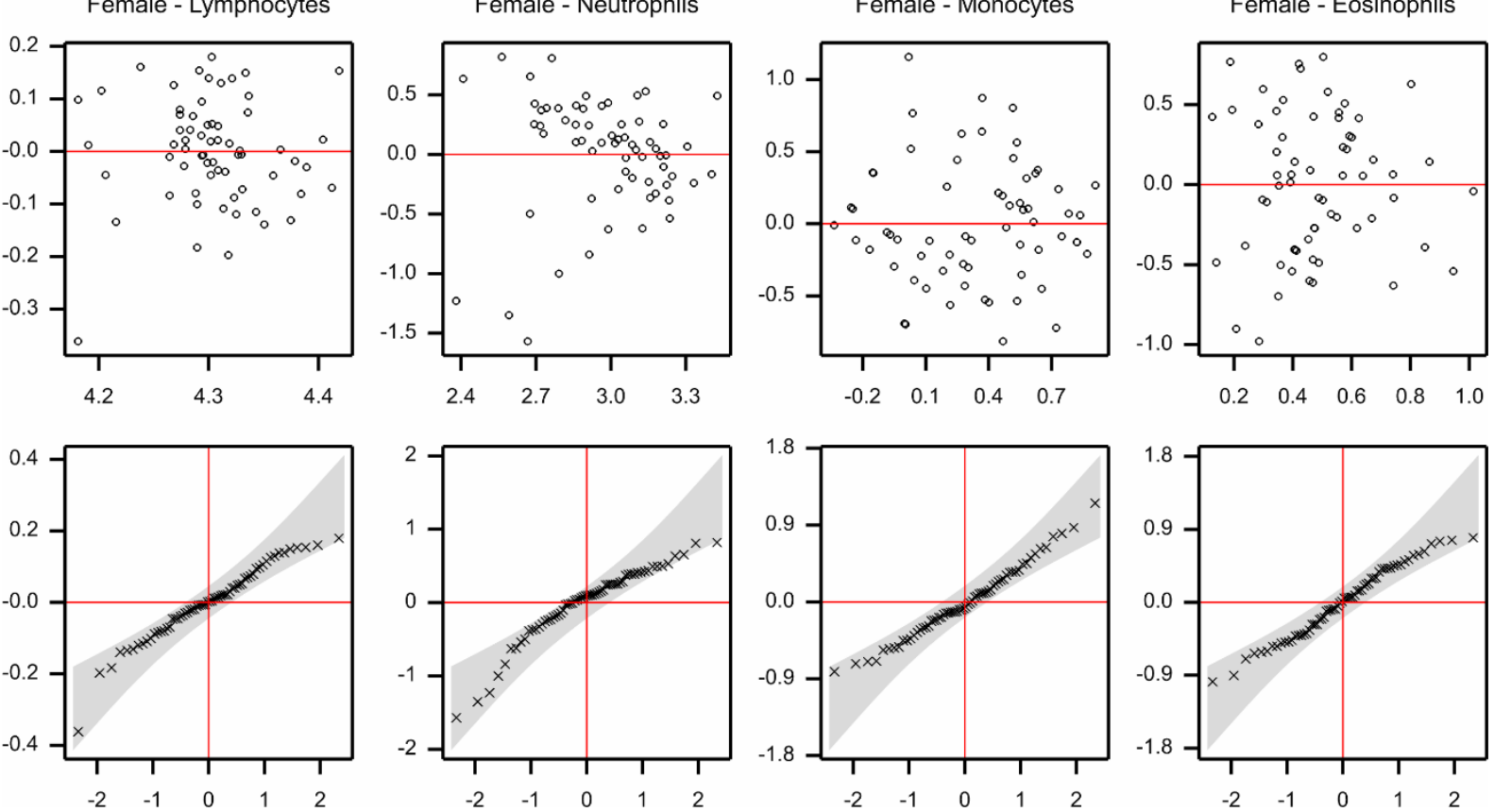

Figure 5 Residuals along the $y$-axis versus fitted values along the $x$-axis resulting from an analysis of variance for Study $C$ on log transformed cage means and normal probability plots for males (top) and females (bottom). 


\subsection{Summary tables and figures}

Cage means on the original and on the log scale for Study A are given in Appendix 1 and Appendix 2, while Appendix 3 and Appendix 4 present these for Study C. Summary tables, on the original nontransformed scale, of number of observations, means, standard deviations and coefficients of variation (\%), classified by the feeding groups, are given in Table 7 for Study A and Table 8 for Study $C$. These tables were obtained by first calculating cage means and then calculating the summary statistics. Plots of feeding group means of Lymphocytes and Neutrophils versus month for Study A are given in Figure 6.
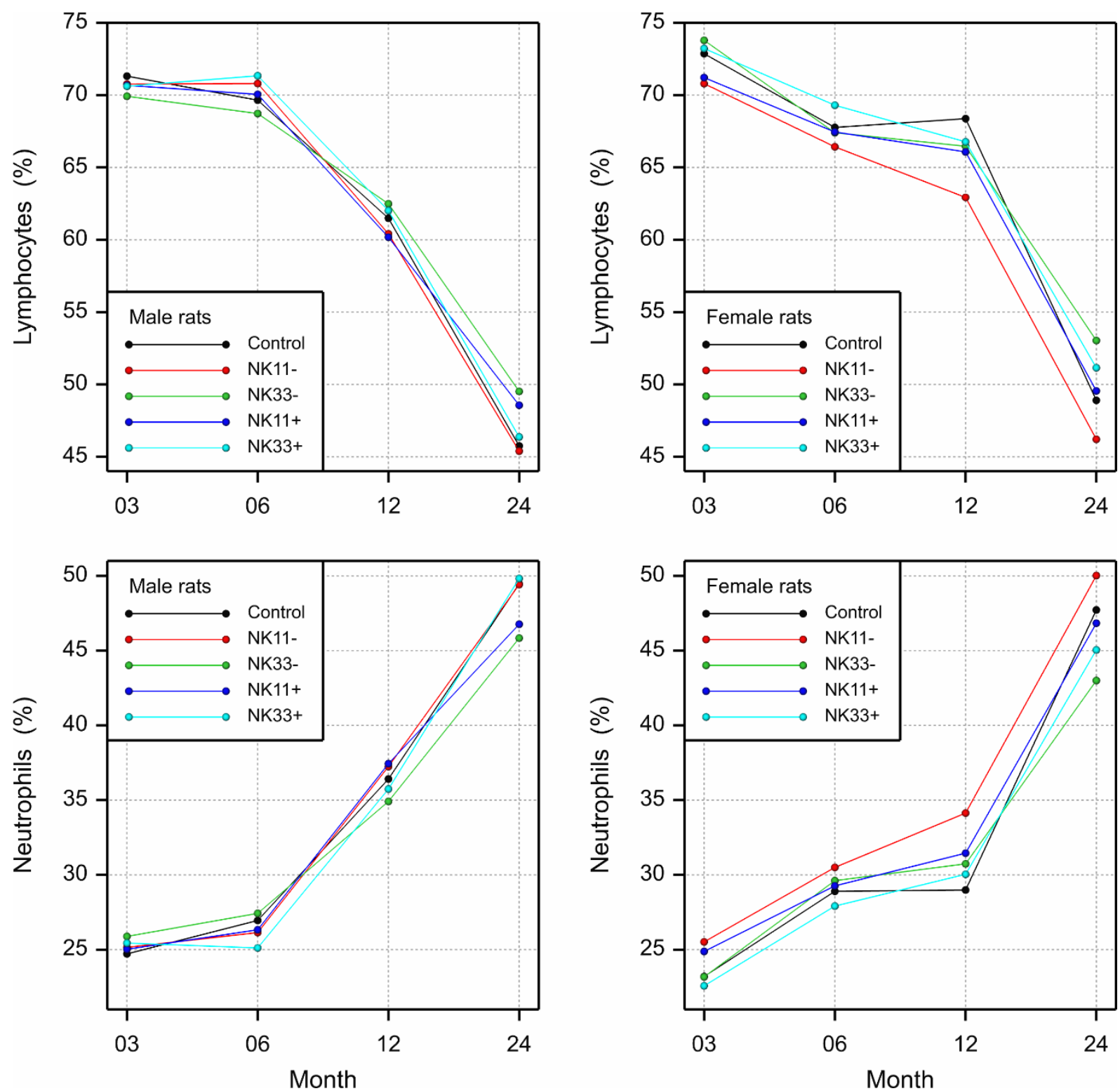

Figure 6 Mean values of Lymphocytes and Neutrophils versus month for each feeding group in Study A for males (left) and females (right). 
Table 7 Summary statistics for male and female rats for Study A classified by the feeding groups: number of cages (N), means (Mean), standard deviations (Sd) and coefficients of variation (CV). The summary statistics are obtained from cage means.

\begin{tabular}{|c|c|c|c|c|c|c|c|c|c|c|c|c|c|c|c|c|c|c|c|c|}
\hline \multirow{2}{*}{$\begin{array}{l}\text { diffWBC } \\
\text { Male } \\
\end{array}$} & \multicolumn{4}{|c|}{ Control } & \multicolumn{4}{|c|}{ NK11- } & \multicolumn{4}{|c|}{ NK33- } & \multicolumn{4}{|c|}{ NK11+ } & \multicolumn{4}{|c|}{ NK33+ } \\
\hline & $\mathrm{N}$ & Mean & $\mathrm{Sd}$ & $\mathrm{CV}$ & $\mathrm{N}$ & Mean & $\mathrm{Sd}$ & $\mathrm{CV}$ & $\mathrm{N}$ & Mean & $\mathrm{Sd}$ & $\mathrm{CV}$ & $\mathrm{N}$ & Mean & $\mathrm{Sd}$ & $\mathrm{CV}$ & $\mathrm{N}$ & Mean & $\mathrm{Sd}$ & $\mathrm{CV}$ \\
\hline Lymphocytes-03 & 20 & 71.3 & 4.1 & 5.8 & 20 & 70.8 & 5.7 & 8.0 & 19 & 71.4 & 5.2 & 7.3 & 20 & 70.7 & 5.9 & 8.3 & 20 & 70.6 & 8.5 & 12.0 \\
\hline Lymphocytes-06 & 20 & 69.7 & 5.2 & 7.5 & 20 & 70.8 & 4.6 & 6.4 & 20 & 68.7 & 5.3 & 7.7 & 20 & 70.0 & 6.5 & 9.3 & 20 & 71.3 & 4.3 & 6.1 \\
\hline Lymphocytes-12 & 20 & 61.5 & 6.0 & 9.8 & 20 & 60.5 & 5.3 & 8.8 & 20 & 62.4 & 6.0 & 9.6 & 21 & 60.2 & 9.6 & 15.9 & 20 & 62.0 & 5.5 & 8.9 \\
\hline Lymphocytes-24 & 21 & 47.1 & 14.7 & 31.2 & 23 & 45.1 & 12.0 & 26.7 & 21 & 49.6 & 7.8 & 15.6 & 23 & 48.8 & 12.5 & 25.5 & 19 & 46.2 & 15.0 & 32.5 \\
\hline Neutrophils-03 & 20 & 24.7 & 4.2 & 16.9 & 20 & 25.1 & 5.7 & 22.6 & 20 & 25.9 & 8.5 & 32.7 & 20 & 25.0 & 6.0 & 23.8 & 20 & 25.4 & 8.4 & 33.0 \\
\hline Neutrophils -06 & 20 & 27.0 & 4.9 & 18.0 & 20 & 26.1 & 4.0 & 15.4 & 20 & 27.4 & 5.3 & 19.3 & 20 & 26.3 & 5.8 & 22.0 & 20 & 25.1 & 4.0 & 15.9 \\
\hline Neutrophils -12 & 20 & 36.4 & 5.8 & 15.8 & 20 & 37.1 & 5.5 & 14.9 & 20 & 35.0 & 6.0 & 17.2 & 21 & 37.3 & 9.0 & 24.2 & 20 & 35.7 & 5.6 & 15.6 \\
\hline Neutrophils -24 & 21 & 48.2 & 13.6 & 28.2 & 23 & 49.7 & 11.5 & 23.0 & 21 & 45.6 & 8.2 & 18.1 & 23 & 46.6 & 12.6 & 27.0 & 19 & 50.0 & 14.4 & 28.8 \\
\hline Monocytes -03 & 20 & 1.9 & 0.5 & 24.1 & 20 & 2.2 & 0.8 & 36.0 & 20 & 2.0 & 0.7 & 36.2 & 20 & 2.1 & 0.8 & 41.0 & 20 & 2.0 & 0.6 & 31.7 \\
\hline Monocytes -06 & 20 & 1.2 & 0.5 & 40.9 & 20 & 0.9 & 0.4 & 43.7 & 20 & 1.1 & 0.5 & 46.9 & 20 & 1.0 & 0.5 & 50.3 & 20 & 1.0 & 0.4 & 35.3 \\
\hline Monocytes -12 & 20 & 0.5 & 0.3 & 63.3 & 20 & 0.6 & 0.4 & 61.1 & 20 & 0.7 & 0.4 & 62.3 & 21 & 0.7 & 0.4 & 61.3 & 20 & 0.7 & 0.5 & 73.6 \\
\hline Monocytes -24 & 21 & 3.5 & 2.8 & 80.8 & 23 & 3.7 & 2.1 & 57.4 & 21 & 3.2 & 1.7 & 53.6 & 23 & 3.2 & 1.6 & 51.5 & 19 & 2.8 & 2.3 & 79.9 \\
\hline Eosinophils -03 & 20 & 2.1 & 1.0 & 47.6 & 20 & 1.8 & 1.1 & 62.4 & 20 & 2.2 & 0.9 & 42.9 & 20 & 2.2 & 1.0 & 44.3 & 20 & 2.0 & 1.0 & 53.1 \\
\hline Eosinophils -06 & 20 & 2.2 & 1.2 & 54.5 & 20 & 2.2 & 1.3 & 60.4 & 20 & 2.8 & 1.2 & 45.3 & 20 & 2.6 & 1.7 & 62.6 & 20 & 2.5 & 0.8 & 33.5 \\
\hline Eosinophils -12 & 20 & 1.6 & 1.1 & 68.0 & 20 & 1.8 & 1.1 & 61.1 & 20 & 1.9 & 0.7 & 39.5 & 21 & 1.8 & 1.4 & 75.6 & 20 & 1.6 & 0.8 & 50.5 \\
\hline Eosinophils -24 & 21 & 1.2 & 0.9 & 78.0 & 23 & 1.4 & 1.0 & 71.1 & 21 & 1.6 & 0.8 & 50.8 & 23 & 1.4 & 1.2 & 83.1 & 19 & 1.0 & 1.0 & 100.1 \\
\hline
\end{tabular}

\begin{tabular}{|c|c|c|c|c|c|c|c|c|c|c|c|c|c|c|c|c|c|c|c|c|}
\hline \multirow{2}{*}{$\begin{array}{l}\text { diffWBC } \\
\text { Female }\end{array}$} & \multicolumn{4}{|c|}{ Control } & \multicolumn{4}{|c|}{ NK11- } & \multicolumn{4}{|c|}{ NK33- } & \multicolumn{4}{|c|}{ NK11+ } & \multicolumn{4}{|c|}{ NK33+ } \\
\hline & $\mathrm{N}$ & Mean & $\mathrm{Sd}$ & $\mathrm{CV}$ & $\mathrm{N}$ & Mean & $\mathrm{Sd}$ & $\mathrm{CV}$ & $\mathrm{N}$ & Mean & $\mathrm{Sd}$ & $\mathrm{CV}$ & $\mathrm{N}$ & Mean & $\mathrm{Sd}$ & $\mathrm{CV}$ & $\mathrm{N}$ & Mean & $\mathrm{Sd}$ & $\mathrm{CV}$ \\
\hline Lymphocytes-03 & 20 & 72.9 & 7.5 & 10.3 & 20 & 71.9 & 7.2 & 10.0 & 20 & 73.8 & 6.7 & 9.1 & 20 & 71.2 & 6.5 & 9.2 & 20 & 73.2 & 7.0 & 9.6 \\
\hline Lymphocytes-06 & 20 & 67.7 & 4.3 & 6.3 & 20 & 67.5 & 4.3 & 6.4 & 20 & 67.4 & 3.9 & 5.8 & 20 & 67.5 & 3.5 & 5.2 & 20 & 69.3 & 4.5 & 6.5 \\
\hline Lymphocytes-12 & 20 & 68.4 & 7.4 & 10.8 & 21 & 62.8 & 6.4 & 10.3 & 22 & 66.5 & 9.0 & 13.6 & 21 & 66.2 & 6.7 & 10.1 & 20 & 66.7 & 6.6 & 9.9 \\
\hline Lymphocytes-24 & 17 & 49.6 & 14.3 & 28.8 & 17 & 46.7 & 11.6 & 24.9 & 20 & 52.9 & 8.1 & 15.2 & 22 & 47.3 & 13.5 & 28.6 & 22 & 50.0 & 13.2 & 26.4 \\
\hline Neutrophils-03 & 20 & 23.2 & 6.4 & 27.7 & 20 & 24.4 & 7.1 & 28.9 & 20 & 23.2 & 6.6 & 28.7 & 20 & 24.9 & 6.0 & 24.1 & 20 & 22.6 & 6.4 & 28.5 \\
\hline Neutrophils -06 & 20 & 29.0 & 4.3 & 14.7 & 20 & 29.5 & 4.1 & 13.8 & 20 & 29.6 & 4.1 & 14.0 & 20 & 29.3 & 3.8 & 12.9 & 20 & 27.9 & 4.3 & 15.5 \\
\hline Neutrophils -12 & 20 & 29.0 & 7.3 & 25.3 & 21 & 34.3 & 6.5 & 18.8 & 22 & 30.7 & 9.1 & 29.6 & 21 & 31.3 & 6.9 & 22.2 & 20 & 30.1 & 6.1 & 20.1 \\
\hline Neutrophils - 24 & 17 & 46.8 & 13.9 & 29.6 & 17 & 49.8 & 10.9 & 22.0 & 20 & 42.9 & 8.5 & 19.9 & 22 & 48.8 & 11.7 & 24.1 & 22 & 46.2 & 13.4 & 29.0 \\
\hline
\end{tabular}




\begin{tabular}{|c|c|c|c|c|c|c|c|c|c|c|c|c|c|c|c|c|c|c|c|c|}
\hline Monocytes -03 & 20 & 1.9 & 0.7 & 37.6 & 20 & 1.7 & 0.7 & 41.5 & 20 & 1.7 & 0.6 & 34.2 & 20 & 2.0 & 0.9 & 42.7 & 20 & 2.0 & 0.7 & 36.2 \\
\hline Monocytes -06 & 20 & 1.1 & 0.6 & 53.7 & 20 & 0.9 & 0.6 & 75.4 & 20 & 1.0 & 0.6 & 60.7 & 20 & 0.9 & 0.4 & 38.6 & 20 & 0.7 & 0.4 & 59.1 \\
\hline Monocytes -12 & 20 & 0.7 & 0.9 & 128.0 & 21 & 0.8 & 0.6 & 72.9 & 22 & 0.9 & 0.7 & 73.1 & 21 & 0.8 & 0.9 & 104.5 & 20 & 1.0 & 0.9 & 91.6 \\
\hline Monocytes -24 & 17 & 2.3 & 2.2 & 94.4 & 17 & 2.2 & 1.4 & 66.0 & 20 & 2.4 & 1.4 & 59.8 & 22 & 2.7 & 2.5 & 90.0 & 22 & 2.4 & 1.6 & 66.1 \\
\hline Eosinophils -03 & 20 & 2.1 & 1.2 & 59.0 & 20 & 2.0 & 1.2 & 61.8 & 20 & 1.3 & 0.8 & 60.5 & 20 & 1.9 & 0.8 & 42.1 & 20 & 2.2 & 1.1 & 50.5 \\
\hline Eosinophils -06 & 20 & 2.2 & 0.8 & 33.9 & 20 & 2.2 & 0.9 & 38.7 & 20 & 2.0 & 1.0 & 49.6 & 20 & 2.3 & 0.8 & 33.0 & 20 & 2.1 & 1.2 & 58.0 \\
\hline Eosinophils -12 & 20 & 1.9 & 0.7 & 36.0 & 21 & 2.2 & 1.2 & 57.2 & 22 & 1.9 & 1.0 & 53.6 & 21 & 1.6 & 0.6 & 34.9 & 20 & 2.2 & 0.7 & 33.6 \\
\hline Eosinophils -24 & 17 & 1.2 & 0.7 & 53.8 & 17 & 1.4 & 0.9 & 68.0 & 20 & 1.8 & 1.1 & 61.3 & 22 & 1.2 & 0.7 & 60.6 & 22 & 1.4 & 0.8 & 60.1 \\
\hline
\end{tabular}

Table 8 Summary statistics for male and female rats for Study C classified by the feeding groups: means (Mean), standard deviations (Sd) and coefficients of variation (CV). The summary statistics are obtained from cage means. All means are based on 8 cages.

\begin{tabular}{|c|c|c|c|c|c|c|c|c|c|c|c|c|c|c|c|c|c|c|c|c|c|c|c|c|}
\hline \multirow{2}{*}{$\begin{array}{l}\text { diffWBC } \\
\text { Male }(\mathrm{N}=8)\end{array}$} & \multicolumn{3}{|c|}{ Con50 } & \multicolumn{3}{|c|}{ NK11-/50 } & \multicolumn{3}{|c|}{ NK50- } & \multicolumn{3}{|c|}{ NK11+/50 } & \multicolumn{3}{|c|}{ NK50+ } & \multicolumn{3}{|c|}{ Con33 } & \multicolumn{3}{|c|}{ NK33- } & \multicolumn{3}{|c|}{ NK33+ } \\
\hline & Mean & $\mathrm{Sd}$ & $\mathrm{CV}$ & Mean & $\mathrm{Sd}$ & $\mathrm{CV}$ & Mean & $\mathrm{Sd}$ & $\mathrm{CV}$ & Mean & $\mathrm{Sd}$ & $\mathrm{CV}$ & Mean & $\mathrm{Sd}$ & $\mathrm{CV}$ & Mean & $\mathrm{Sd}$ & $\mathrm{CV}$ & Mean & $\mathrm{Sd}$ & $\mathrm{CV}$ & Mean & $\mathrm{Sd}$ & CV \\
\hline Lymphocytes & 78.1 & 8.9 & 11.3 & 79.4 & 10.7 & 13.5 & 82.7 & 12.6 & 15.2 & 74.4 & 10.7 & 14.4 & 73.8 & 12.4 & 16.8 & 67.0 & 11.4 & 17.0 & 74.1 & 10.7 & 14.4 & 71.9 & 12.9 & 18.0 \\
\hline Neutrophils & 18.8 & 8.8 & 47.0 & 17.1 & 9.6 & 56.1 & 14.2 & 12.3 & 86.0 & 22.0 & 9.5 & 43.4 & 22.4 & 11.1 & 49.4 & 29.2 & 10.4 & 35.5 & 21.3 & 9.5 & 44.6 & 24.4 & 12.5 & 51.3 \\
\hline Monocytes & 1.97 & 0.59 & 29.9 & 2.03 & 1.80 & 88.6 & 1.62 & 1.10 & 67.8 & 2.09 & 1.48 & 70.5 & 2.00 & 1.19 & 59.4 & 1.62 & 1.22 & 74.9 & 2.78 & 1.63 & 58.5 & 1.78 & 0.83 & 46.5 \\
\hline Eosinophils & 1.16 & 0.42 & 36.4 & 1.47 & 0.75 & 51.0 & 1.47 & 0.83 & 56.4 & 1.50 & 0.50 & 33.3 & 1.84 & 1.47 & 79.7 & 2.19 & 2.06 & 94.4 & 1.84 & 0.74 & 40.3 & 1.87 & 0.69 & 37.0 \\
\hline
\end{tabular}

\begin{tabular}{|c|c|c|c|c|c|c|c|c|c|c|c|c|c|c|c|c|c|c|c|c|c|c|c|c|}
\hline diffWBC & \multicolumn{3}{|c|}{ Con50 } & \multicolumn{3}{|c|}{ NK11-/50 } & \multicolumn{3}{|c|}{ NK50- } & \multicolumn{3}{|c|}{ NK11+/50 } & \multicolumn{3}{|c|}{ NK50+ } & \multicolumn{3}{|c|}{ Con33 } & \multicolumn{3}{|c|}{ NK33- } & \multicolumn{3}{|c|}{ NK33+ } \\
\hline Female $(\mathrm{N}=8)$ & Mean & $\mathrm{Sd}$ & $\mathrm{CV}$ & Mean & $\mathrm{Sd}$ & $\mathrm{CV}$ & Mean & $\mathrm{Sd}$ & $\mathrm{CV}$ & Mean & $\mathrm{Sd}$ & $\mathrm{CV}$ & Mean & $\mathrm{Sd}$ & $\mathrm{CV}$ & Mean & $\mathrm{Sd}$ & $\mathrm{CV}$ & Mean & $\mathrm{Sd}$ & $\mathrm{CV}$ & Mean & $\mathrm{Sd}$ & CV \\
\hline Lymphocytes & 72.7 & 11.4 & 15.7 & 74.0 & 7.4 & 10.0 & \begin{tabular}{|l|}
76.4 \\
\end{tabular} & 7.0 & 9.2 & \begin{tabular}{|l|}
72.4 \\
\end{tabular} & 1.8 & 2.4 & 74.8 & 9.7 & 12.9 & 80.5 & 8.3 & 10.3 & 73.8 & 6.8 & 9.2 & 73.2 & 7.5 & 10.2 \\
\hline Neutrophils & 24.8 & 10.7 & 43.3 & 23.0 & 7.1 & 31.0 & 21.0 & 7.1 & 33.6 & 25.3 & 2.5 & 10.1 & 21.9 & 9.4 & 43.2 & 17.4 & 7.6 & 43.5 & 23.1 & 7.7 & 33.2 & 24.4 & 7.4 & 30.3 \\
\hline Monocytes & 1.16 & 1.00 & 86.4 & 1.41 & 1.07 & 76.0 & 1.31 & 0.74 & 56.5 & 1.09 & 0.93 & 84.6 & 1.34 & 0.79 & 58.8 & 0.88 & 0.76 & 86.4 & 1.53 & 0.90 & 58.8 & 0.97 & 0.78 & 80.9 \\
\hline Eosinophils & 1.38 & 0.78 & 56.7 & 1.53 & 0.69 & 44.9 & 1.25 & 0.61 & 49.0 & 1.19 & 0.88 & 74.4 & 1.94 & 0.85 & 44.0 & 1.16 & 0.65 & 56.5 & 1.50 & 1.07 & 71.3 & 1.44 & 0.94 & 65.6 \\
\hline
\end{tabular}




\section{Statistical analysis}

\subsection{Equivalence testing for study A using historical data}

Figure 7 displays, for the diffWBC endpoints, the ratio of the residual variance in the current G-TwYST study $A$ as a percentage of the residual variance in the historical GRACE study. This indicates that the 12 and 24 month data are in general more variable than those observed after 3 and 6 months. It also shows that even after 3 and 6 months the Lymphocytes data in G-TwYST Study A is (much) more variable than in GRACE resulting in a less useful equivalence analysis.
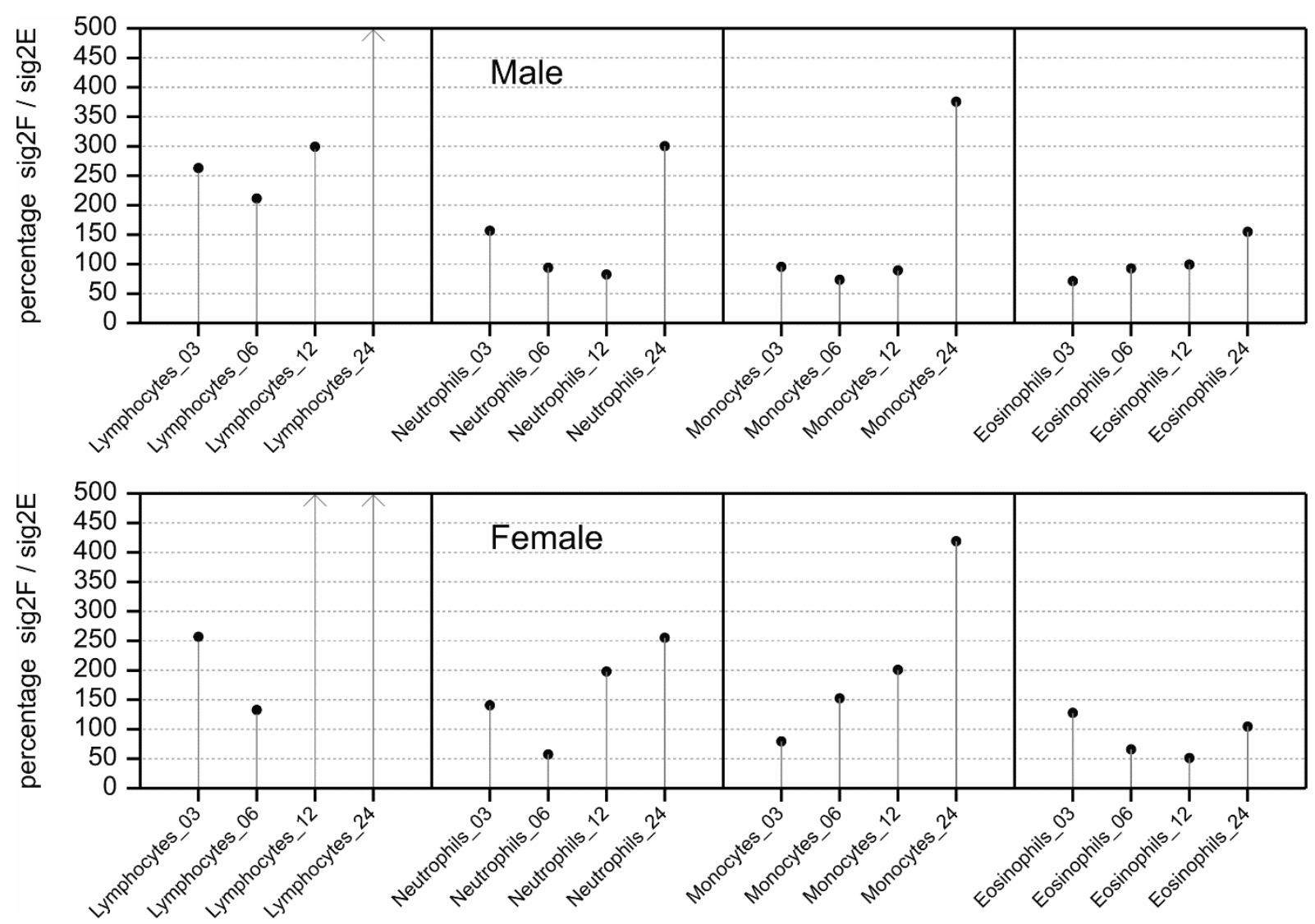

Figure 7 Residual variance (sig2F or $\sigma_{F}^{2}$ ) in the current G-TwYST A study as a percentage of the residual variance (sig2E or $\sigma_{E}^{2}$ ) in the historical GRACE studies for males (top panel) and females (bottom panel) that survived for 24 months.

Preliminary regulatory values $\alpha=0.05, \beta=0.20$ and $n_{0}=20$ were employed, where $n_{0}$ is similar to the number of cages for the diffWBC endpoints (Table 7). The DWE intervals showing the main results of the equivalence tests are given in Figure 8 and Figure 9. The hypothesis of no difference is rejected in case the interval does not contain zero, which is denoted by fuchsia coloured estimates. The non-equivalence hypothesis is rejected when the interval fully lies inside the interval $(-1,1)$. For further interpretation the $95 \%$ confidence intervals for the ratios are given in Figure 10 and Figure 11, and also in Table 9. For most cases where the residual variance in G-TwYST is $150 \%$ or larger than in the reference data there was a failure to prove equivalence, but equivalence was still more likely than not in all cases but one (Females, Control vs. NK11-, N-12). In all cases where the residual variance was less than $150 \%$ compared to the reference data equivalence could be established, except for Eosinophils-03 for Females Control vs NK33-. 
Male Control vs NK11-

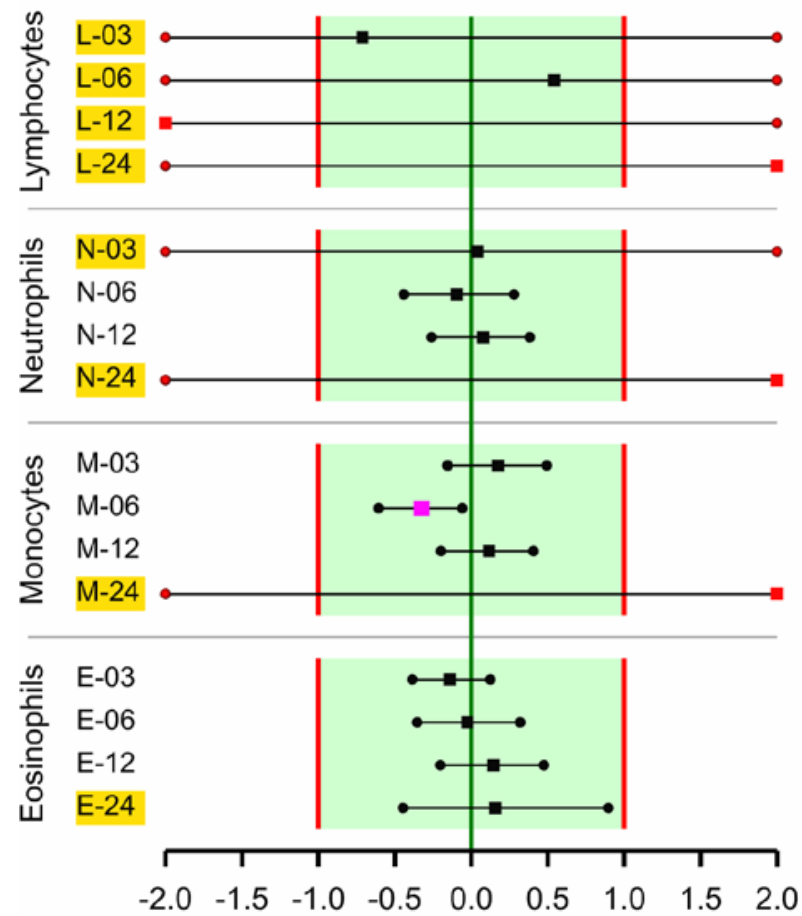

Male Control vs NK33-

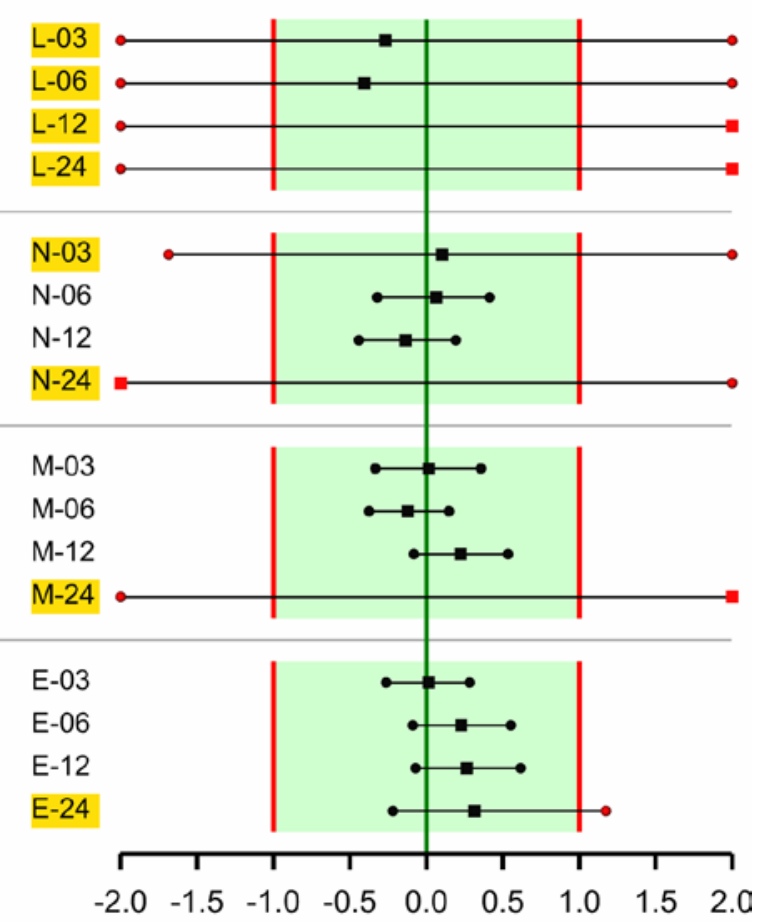

Male Control vs NK11+

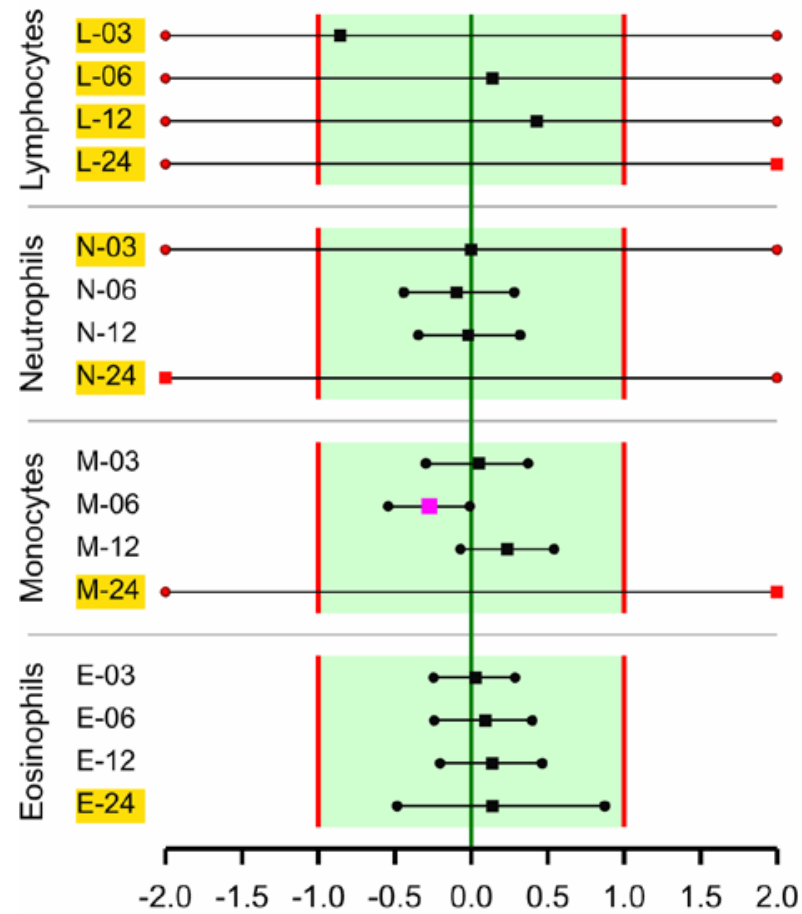

Equivalence Limit Scaled Difference
Male Control vs NK33+

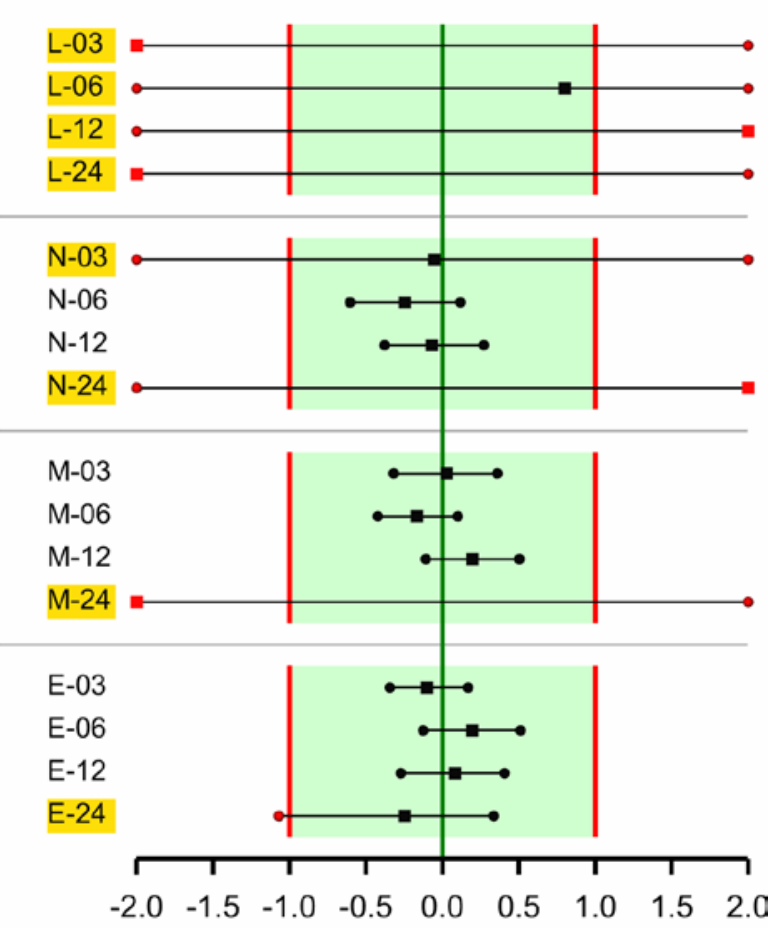

Equivalence Limit Scaled Difference

Figure 8 Equivalence testing of GM feeding groups versus the control feed for males in study A. For estimates (square symbols) on the left of zero the GM feed has a smaller mean than the control feed (see also Table 9). Endpoints labelled with a golden background have a large residual variance compared to the historical studies (variance ratio VR $>150 \%$ ). Fuchsia coloured symbols denote a significant difference. 
Female Control vs NK11-

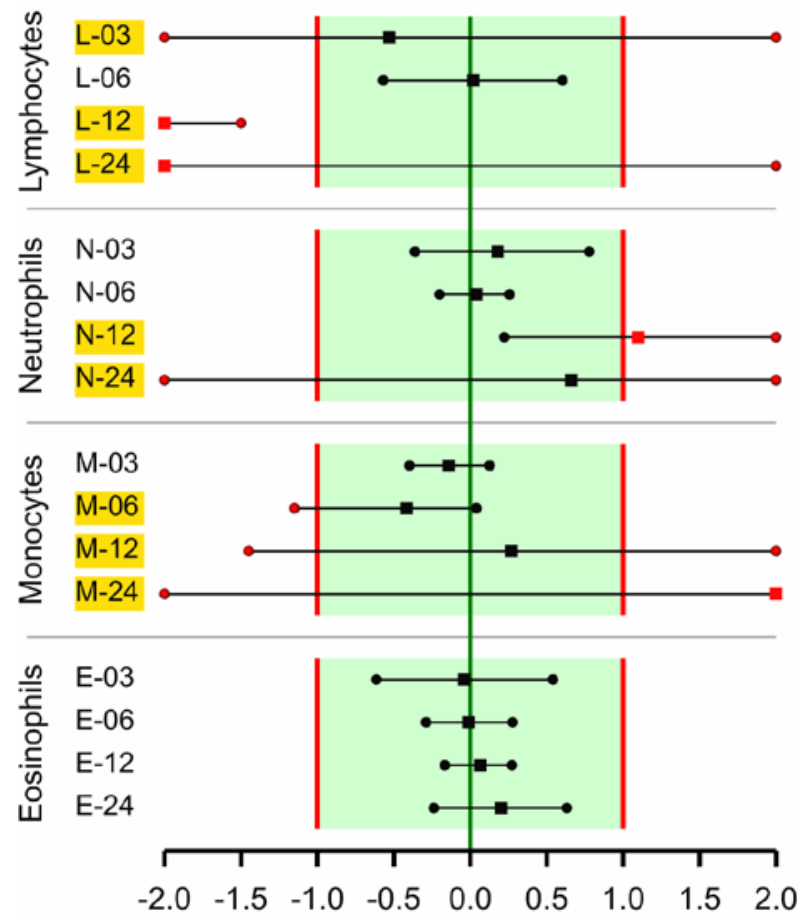

Female Control vs NK11+

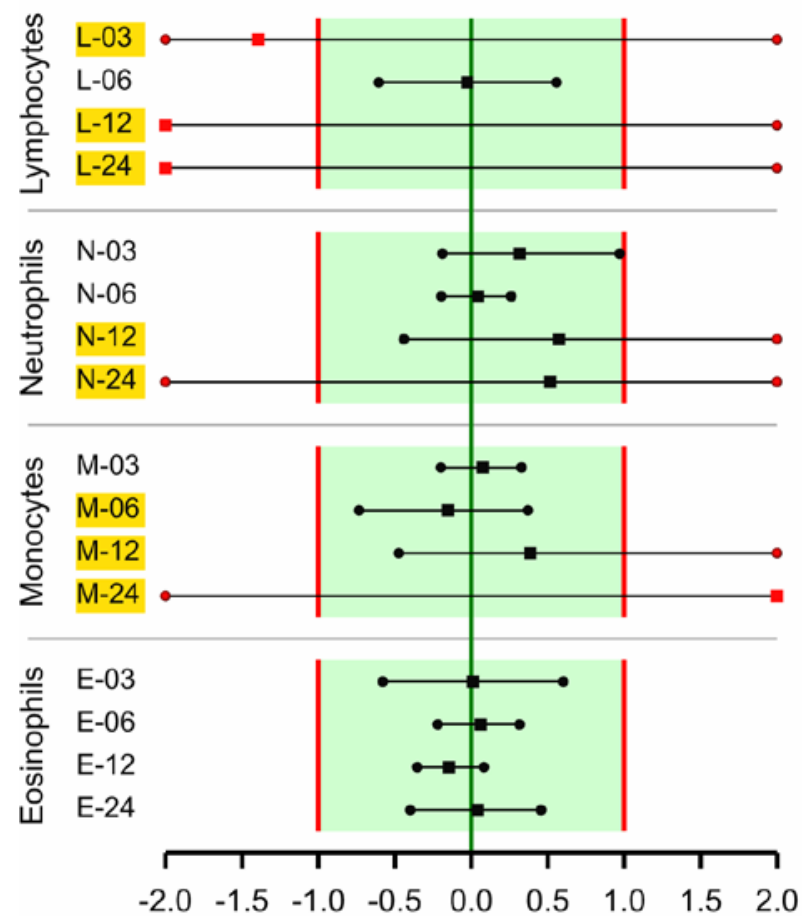

Equivalence Limit Scaled Difference
Female Control vs NK33-

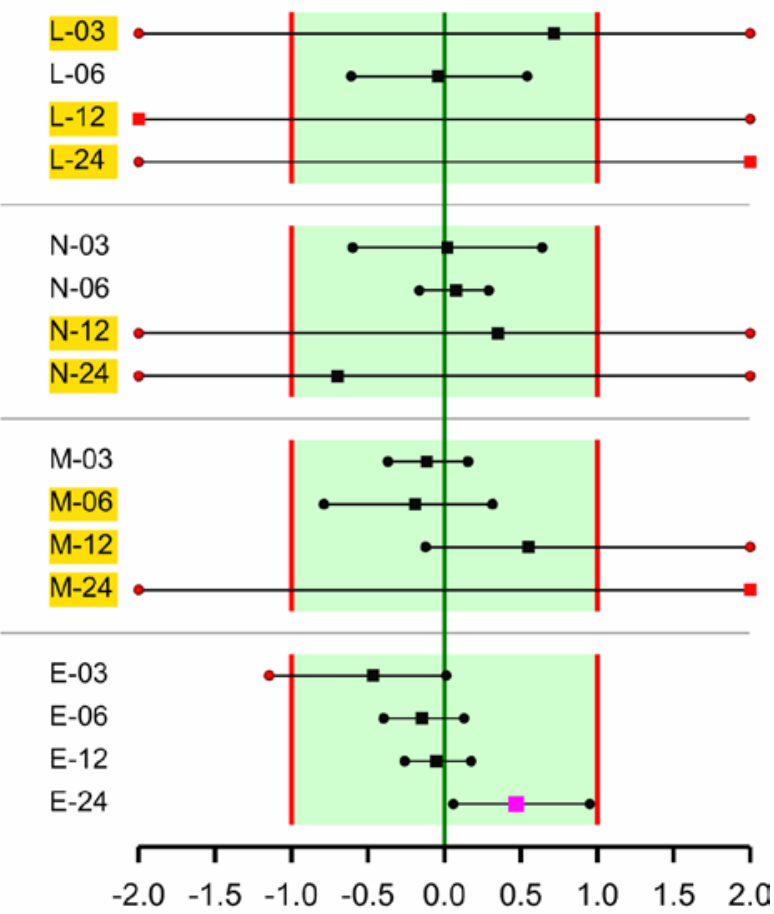

Female Control vs NK33+

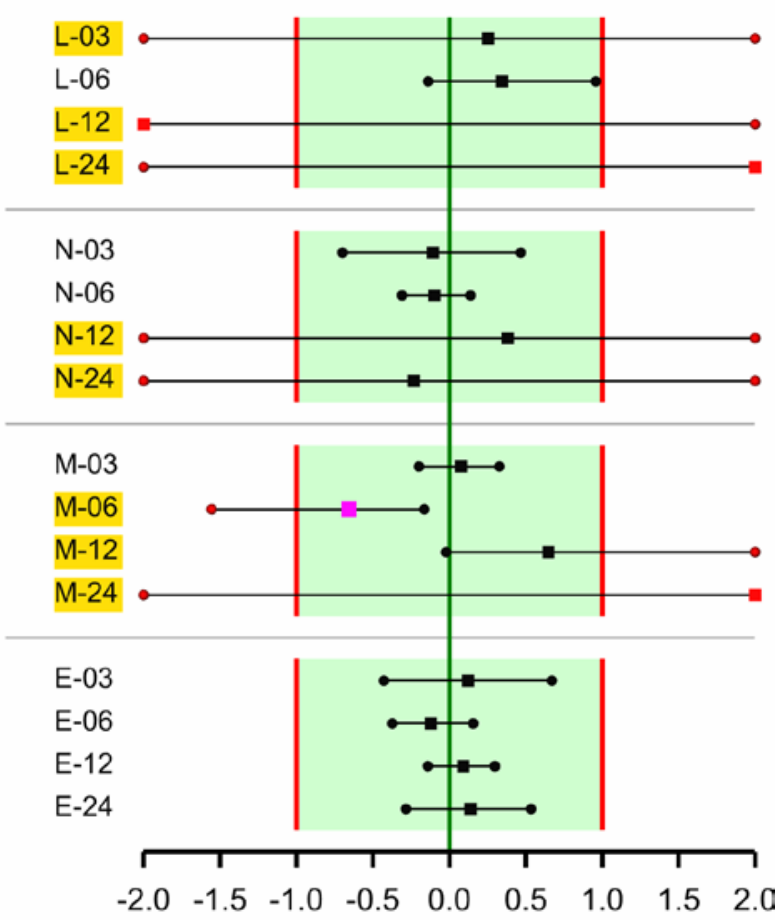

Equivalence Limit Scaled Difference

Figure 9 Equivalence testing of GM feeding groups versus the control feed for females in study A. For estimates (square symbols) on the left of zero the GM feed has a smaller mean than the control feed (see also Table 9). Endpoints labelled with a golden background have a large residual variance compared to the historical studies (variance ratio VR $>150 \%$ ). Fuchsia coloured symbols denote a significant difference. 

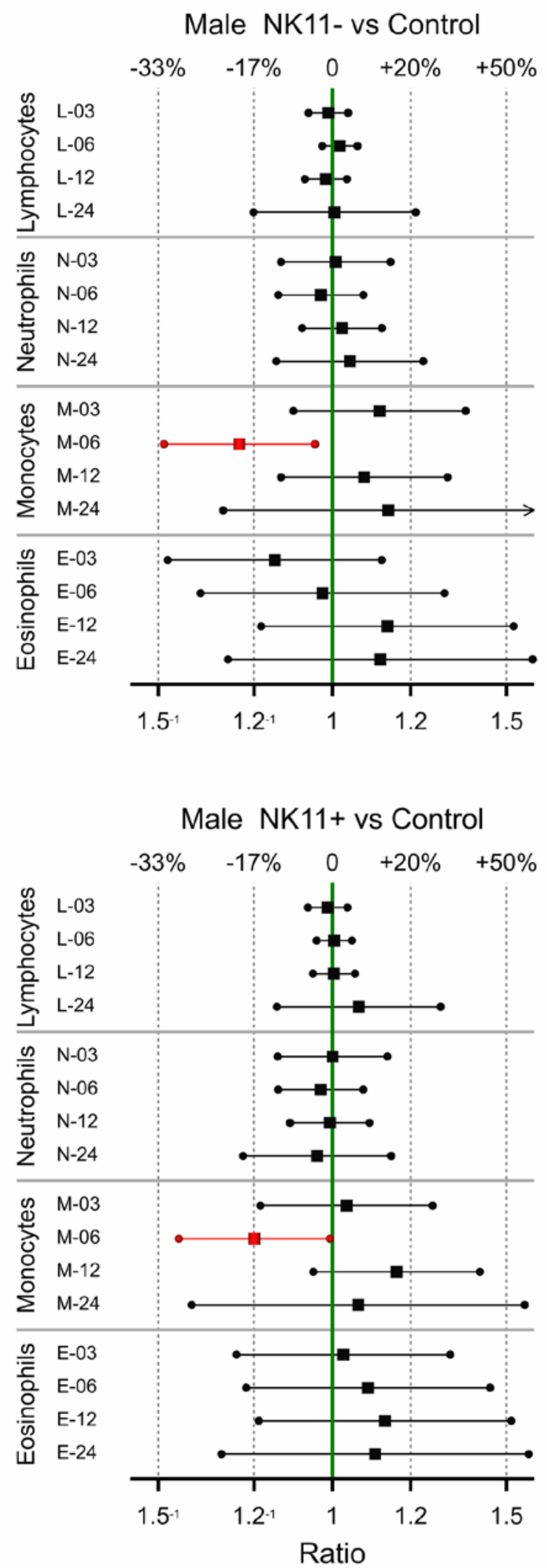
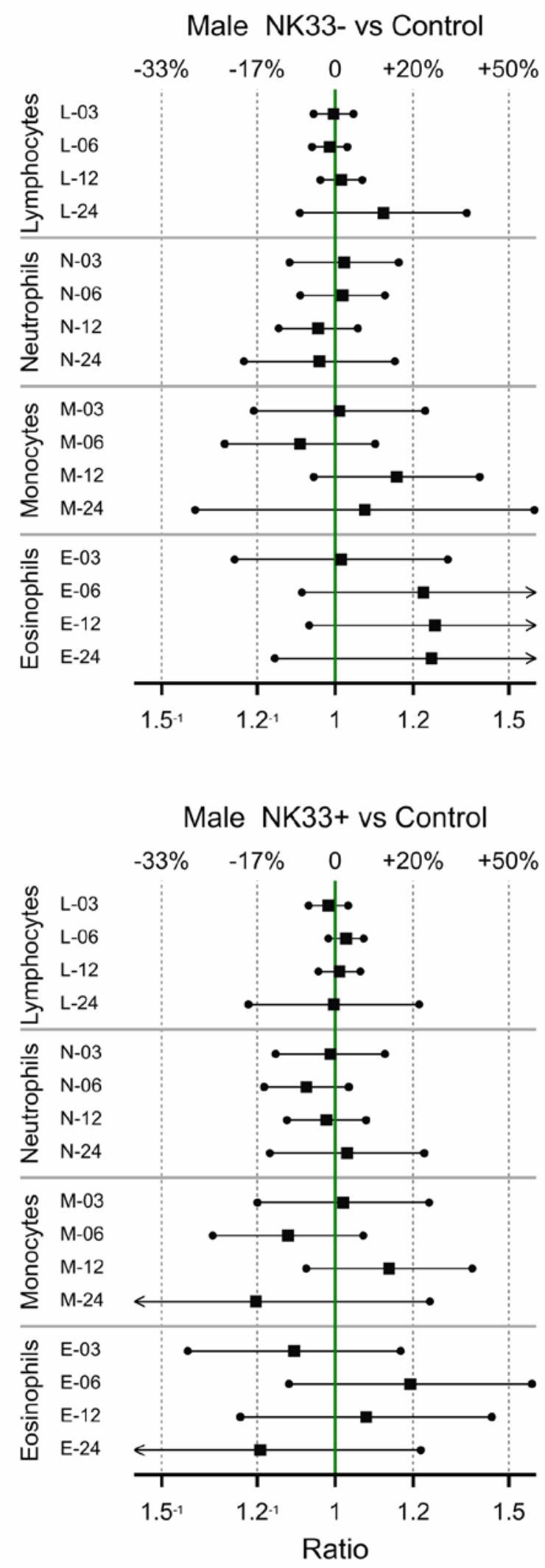

Figure 10 95\% confidence intervals for the four comparisons for males in Study A. Intervals that do not encompass the ratio= 1 line are given in red, these represent significant differences at the $5 \%$ level. 
Female NK11- vs Control

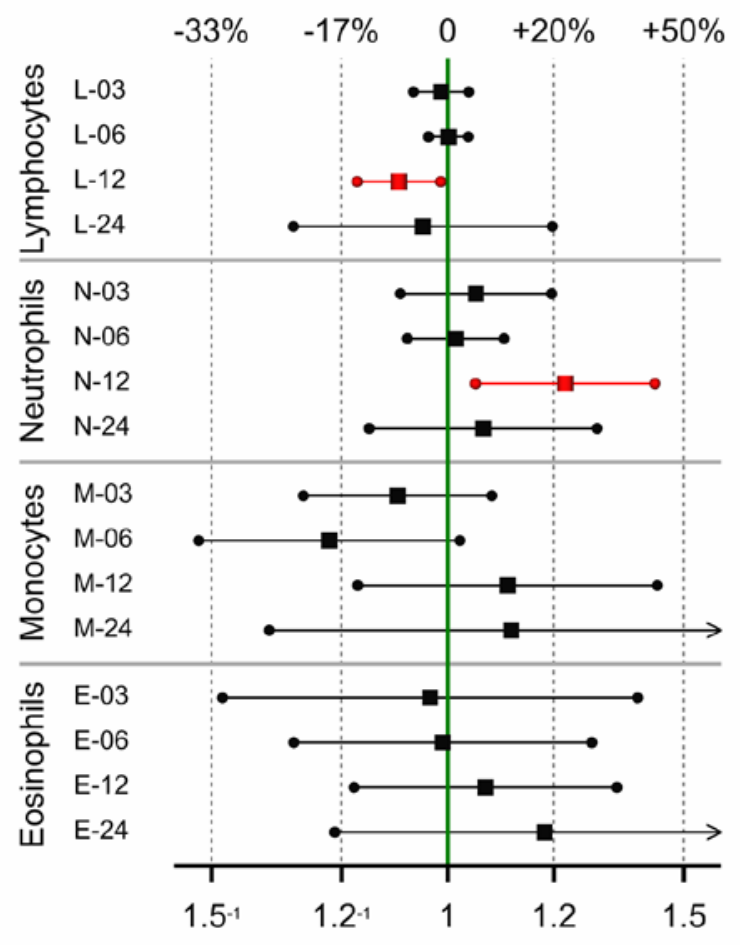

Female NK11+ vs Control

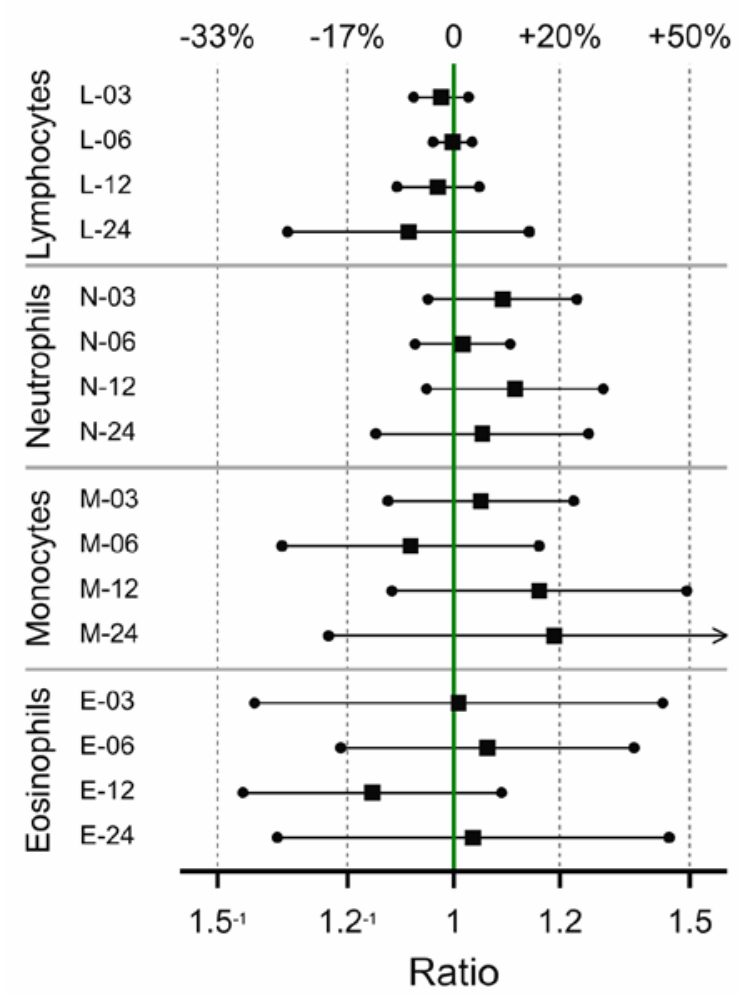

Female NK33- vs Control

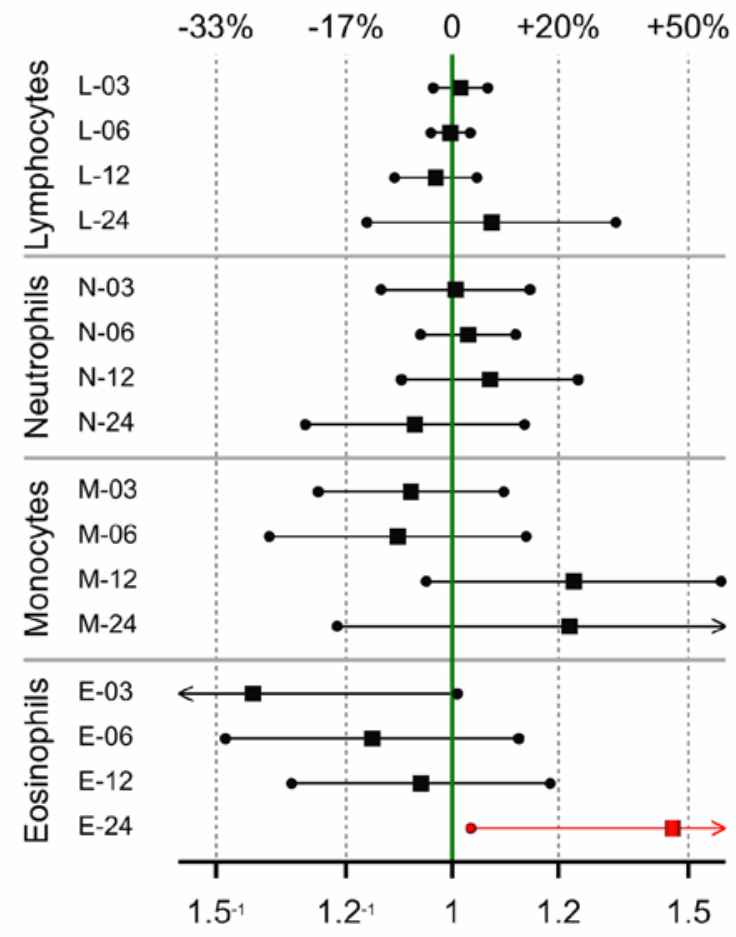

Female NK33+ vs Control

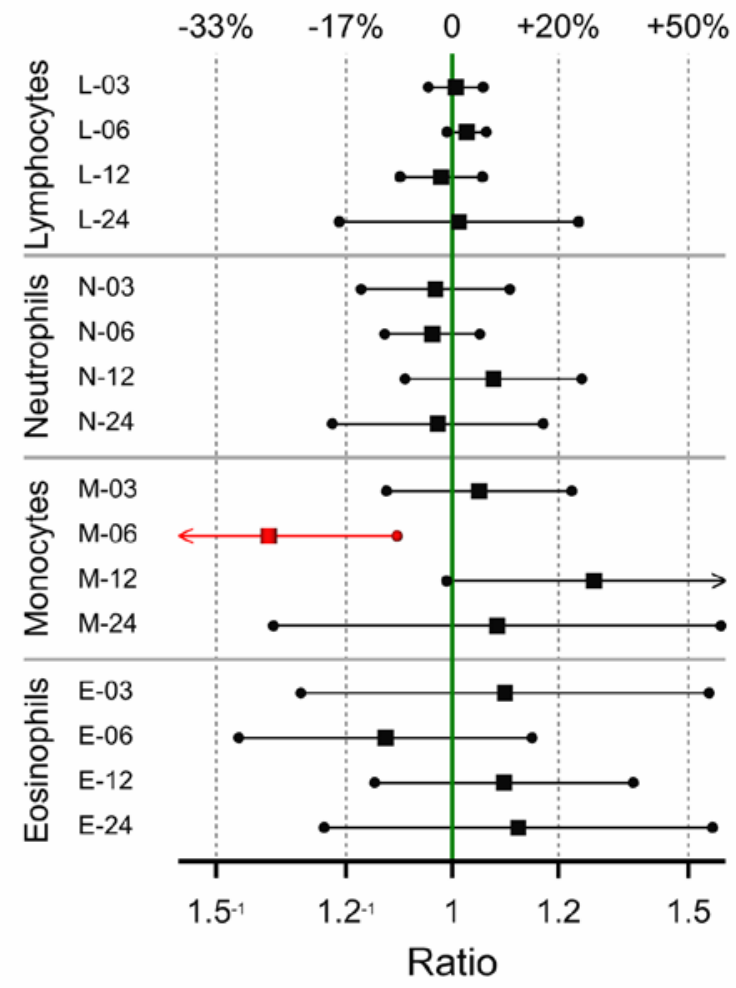

Figure $1195 \%$ confidence intervals for the four comparisons for females in Study A. Intervals that do not encompass the ratio $=1$ line are given in red, these represent significant differences at the $5 \%$ level. 
Male Control vs NK11-

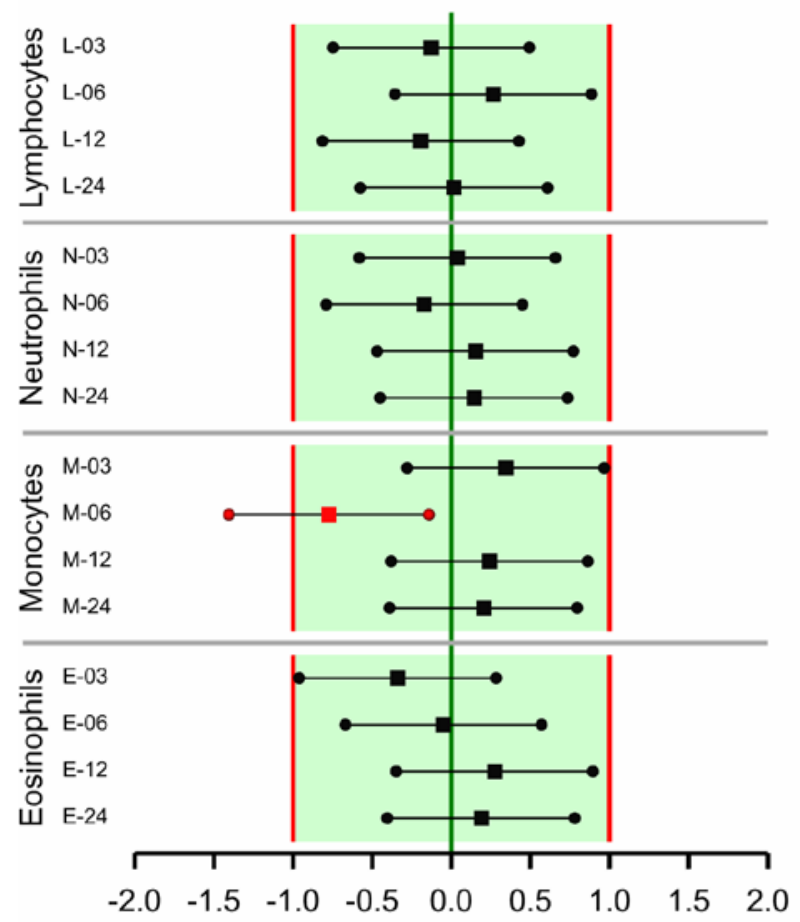

Male Control vs NK11+

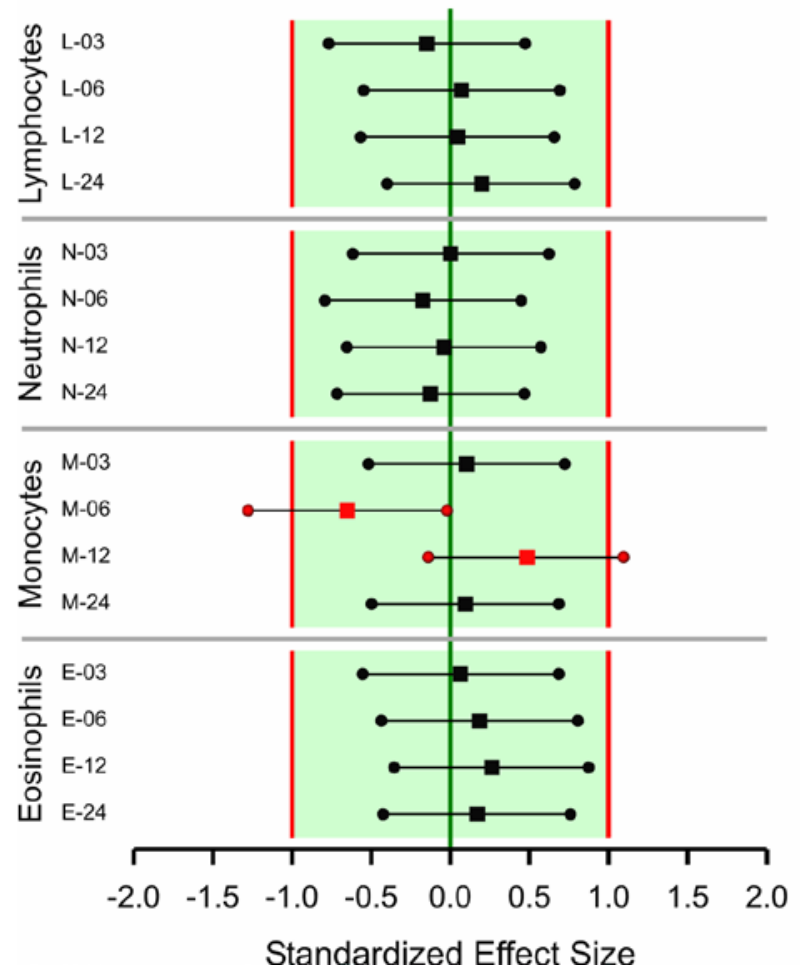

Male Control vs NK33-

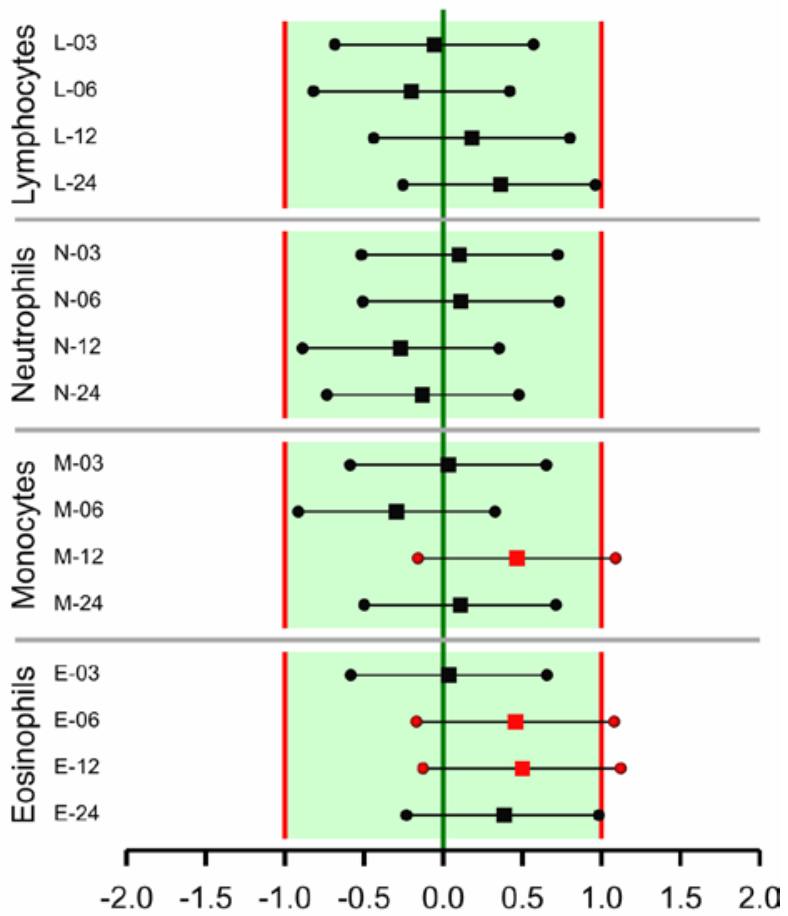

Male Control vs NK33+

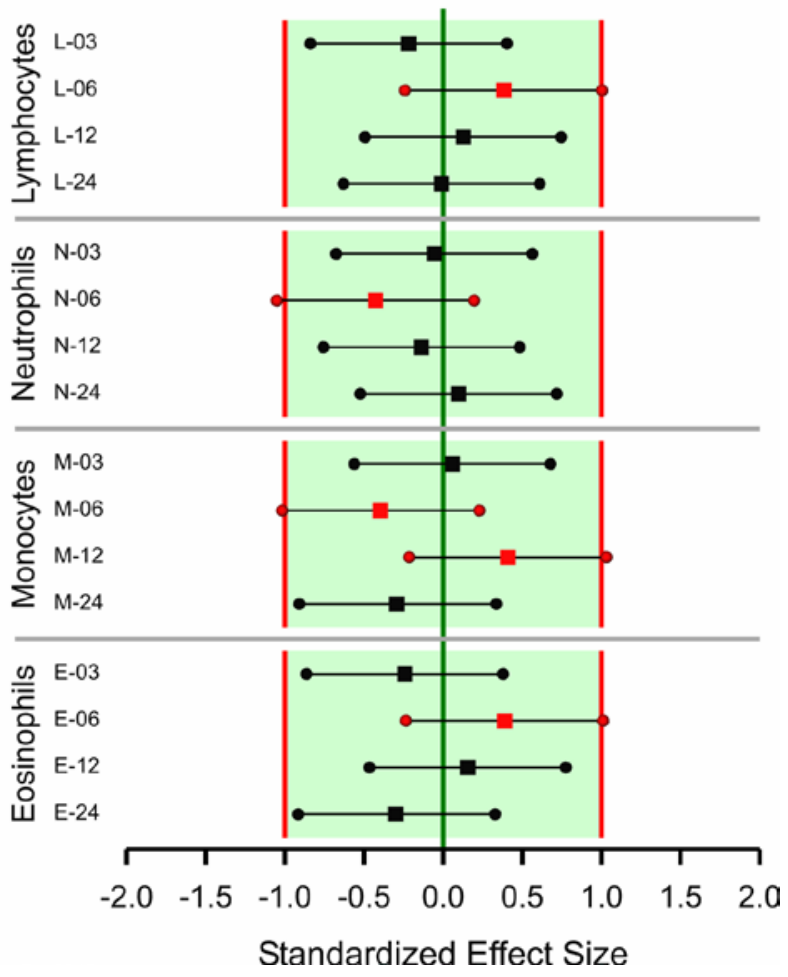

Figure 12 Confidence intervals for Standardized Effect Sized (SES) for male rats in Study A. Intervals that extend beyond the interval $(-1,1)$ are given in red. 
Female Control vs NK11-

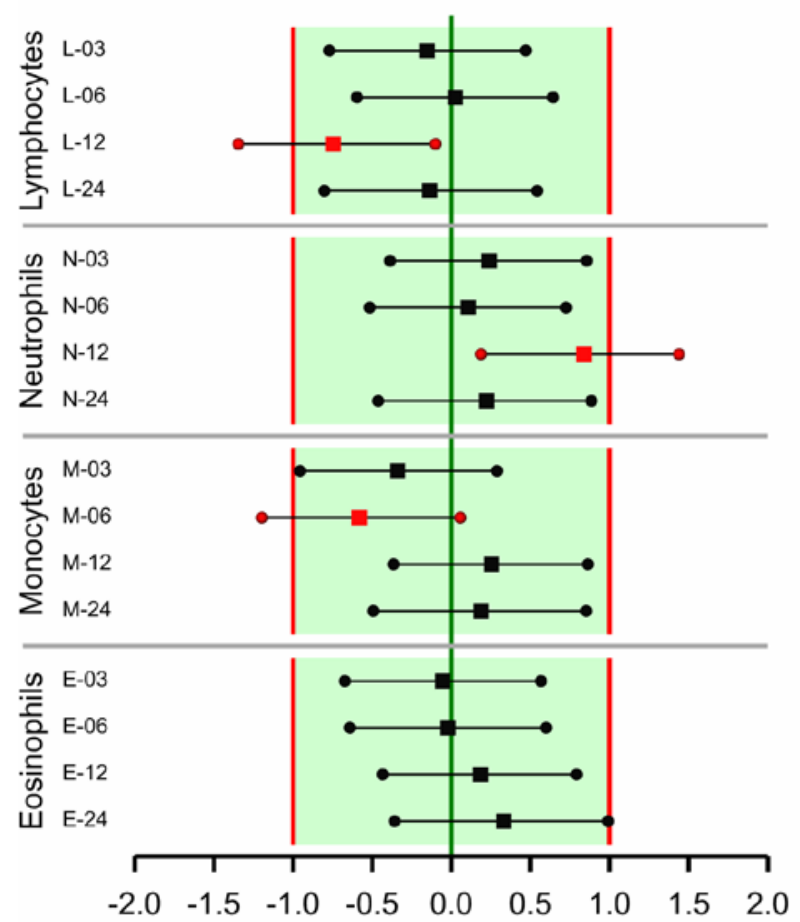

Female Control vs NK11+

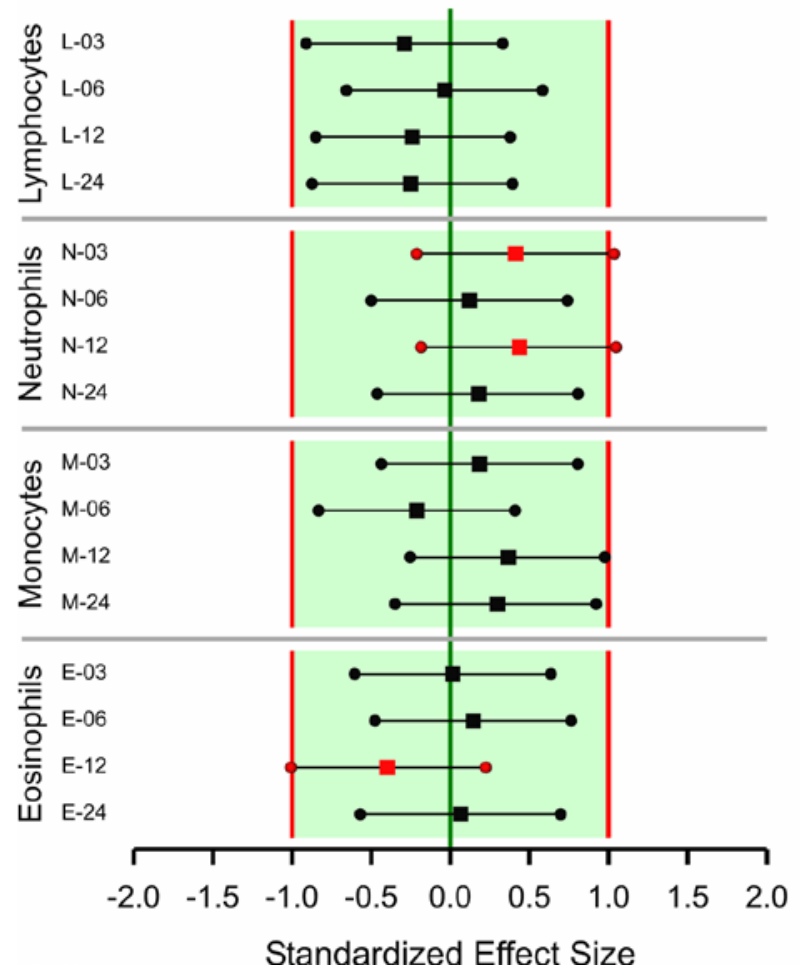

Female Control vs NK33-

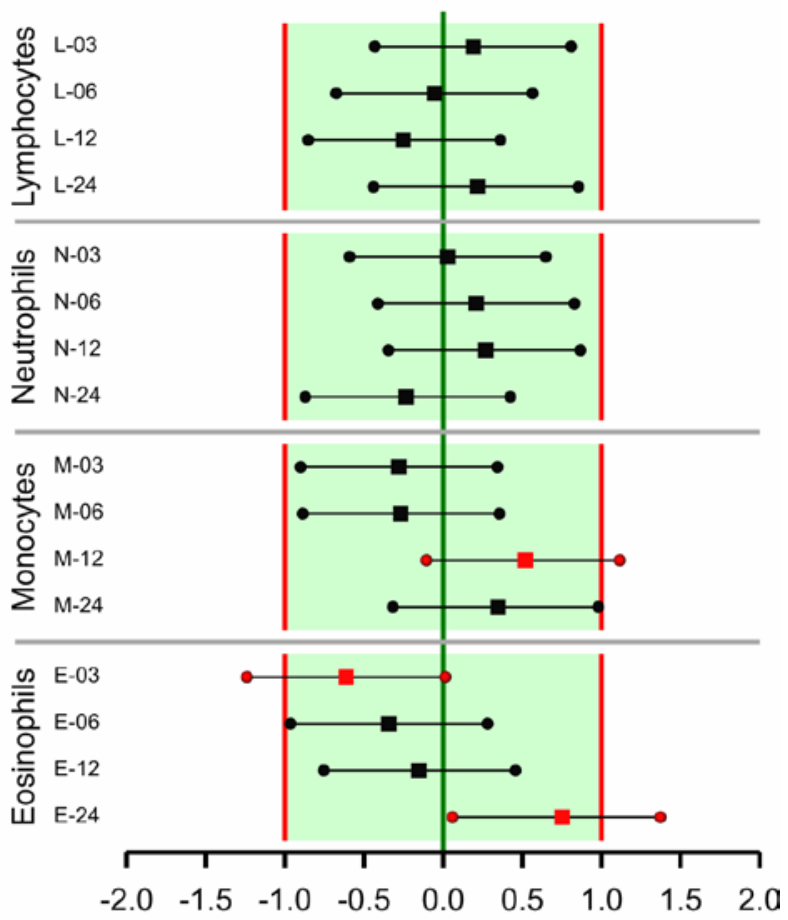

Female Control vs NK33+

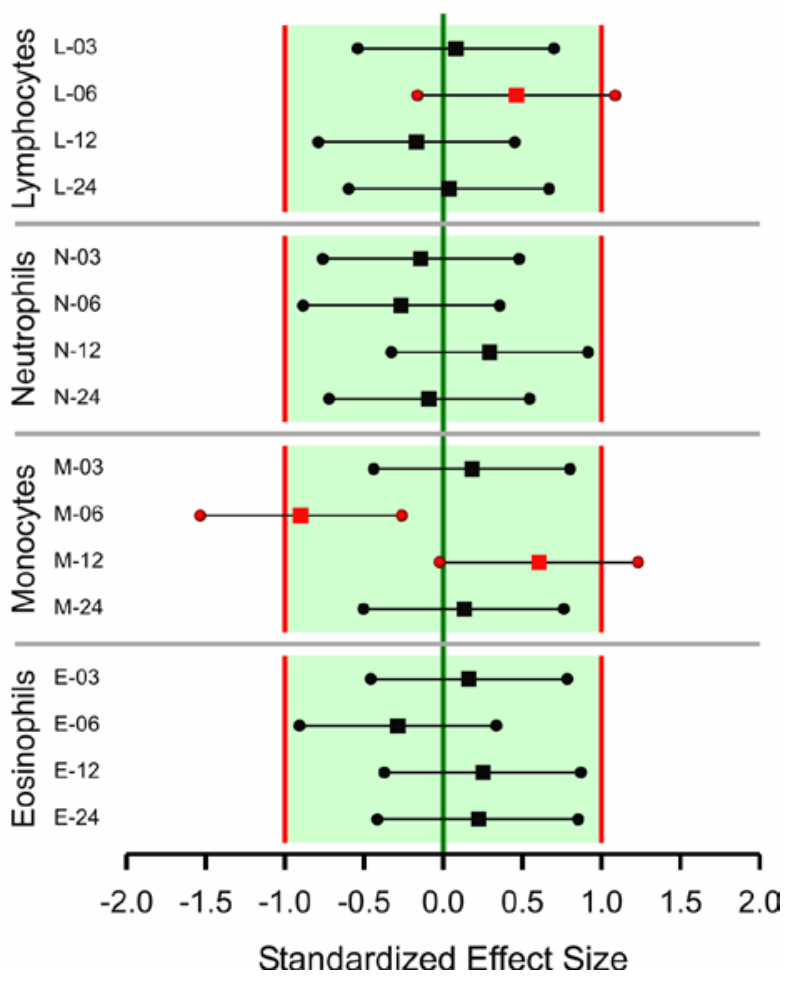

Figure 13 Confidence intervals for Standardized Effect Sized (SES) for female rats in Study A. Intervals that extend beyond the interval $(-1,1)$ are given in red. 
Table $995 \%$ Confidence interval plus estimate for the ratio $\Delta$ of the GMO feeds versus the Control feed for Study A. Intervals are based on an ANOVA with 5 feeding groups. Ratios with corresponding $95 \%$ confidence intervals that do not encompass the value 1 are coloured red; this is equivalent to a significant difference according to a t-test with significance level $5 \%$.

\begin{tabular}{|l|r|r|r|r|r|r|r|r|r|r|r|r|r|}
\hline diffWBC & \multicolumn{3}{|c|}{ NK11- vs Control } & \multicolumn{3}{|c|}{ NK33- vs Control } & \multicolumn{2}{c|}{ NK11+ vs Control } & \multicolumn{3}{c|}{ NK33+ vs Control } \\
\hline Males & Lower & Ratio & Upper & Lower & Ratio & Upper & Lower & Ratio & Upper & Lower & Ratio & Upper \\
\hline Lymphocytes-03 & 0.946 & 0.991 & 1.037 & 0.950 & 0.996 & 1.043 & 0.945 & 0.989 & 1.036 & 0.940 & 0.984 & 1.030 \\
\hline Lymphocytes-06 & 0.977 & 1.018 & 1.060 & 0.947 & 0.987 & 1.028 & 0.964 & 1.005 & 1.047 & 0.984 & 1.025 & 1.068 \\
\hline Lymphocytes-12 & 0.938 & 0.985 & 1.034 & 0.966 & 1.014 & 1.065 & 0.956 & 1.004 & 1.054 & 0.962 & 1.010 & 1.061 \\
\hline Lymphocytes-24 & 0.832 & 1.005 & 1.214 & 0.921 & 1.119 & 1.360 & 0.879 & 1.064 & 1.287 & 0.816 & 0.997 & 1.217 \\
\hline Neutrophils-03 & 0.887 & 1.008 & 1.145 & 0.899 & 1.021 & 1.160 & 0.881 & 1.000 & 1.137 & 0.870 & 0.989 & 1.123 \\
\hline Neutrophils-06 & 0.882 & 0.973 & 1.075 & 0.922 & 1.018 & 1.124 & 0.881 & 0.973 & 1.074 & 0.847 & 0.935 & 1.032 \\
\hline Neutrophils -12 & 0.932 & 1.023 & 1.122 & 0.876 & 0.961 & 1.054 & 0.906 & 0.994 & 1.091 & 0.893 & 0.980 & 1.075 \\
\hline Neutrophils -24 & 0.878 & 1.041 & 1.235 & 0.808 & 0.964 & 1.150 & 0.812 & 0.965 & 1.147 & 0.858 & 1.028 & 1.231 \\
\hline Monocytes -03 & 0.913 & 1.116 & 1.364 & 0.827 & 1.010 & 1.234 & 0.846 & 1.034 & 1.263 & 0.834 & 1.019 & 1.245 \\
\hline Monocytes -06 & 0.676 & 0.805 & 0.960 & 0.773 & 0.921 & 1.098 & 0.699 & 0.834 & 0.994 & 0.751 & 0.895 & 1.067 \\
\hline Monocytes -12 & 0.887 & 1.077 & 1.308 & 0.951 & 1.154 & 1.401 & 0.956 & 1.161 & 1.410 & 0.934 & 1.134 & 1.377 \\
\hline Monocytes -24 & 0.776 & 1.139 & 1.672 & 0.721 & 1.072 & 1.593 & 0.721 & 1.062 & 1.565 & 0.554 & 0.831 & 1.247 \\
\hline Eosinophils -03 & 0.682 & 0.875 & 1.122 & 0.791 & 1.014 & 1.301 & 0.800 & 1.026 & 1.316 & 0.708 & 0.909 & 1.165 \\
\hline Eosinophils -06 & 0.736 & 0.978 & 1.299 & 0.925 & 1.229 & 1.633 & 0.818 & 1.087 & 1.444 & 0.897 & 1.192 & 1.583 \\
\hline Eosinophils -12 & 0.847 & 1.137 & 1.525 & 0.941 & 1.263 & 1.694 & 0.843 & 1.131 & 1.517 & 0.801 & 1.075 & 1.442 \\
\hline Eosinophils -24 & 0.784 & 1.118 & 1.594 & 0.868 & 1.252 & 1.806 & 0.772 & 1.105 & 1.580 & 0.577 & 0.839 & 1.221 \\
\hline
\end{tabular}

\begin{tabular}{|l|r|r|r|r|r|r|r|r|r|r|r|r|r|}
\hline diffWBC & \multicolumn{3}{|c|}{ NK11- vs Control } & \multicolumn{3}{|c|}{ NK33- vs Control } & \multicolumn{3}{|c|}{ NK11+ vs Control } & \multicolumn{3}{c|}{ NK33+ vs Control } \\
\hline Females & Lower & Ratio & Upper & Lower & Ratio & Upper & Lower & Ratio & Upper & Lower & Ratio & Upper \\
\hline Lymphocytes-03 & 0.943 & 0.989 & 1.037 & 0.968 & 1.014 & 1.063 & 0.934 & 0.979 & 1.026 & 0.960 & 1.006 & 1.054 \\
\hline Lymphocytes-06 & 0.968 & 1.001 & 1.036 & 0.964 & 0.997 & 1.031 & 0.965 & 0.998 & 1.032 & 0.991 & 1.025 & 1.060 \\
\hline Lymphocytes-12 & 0.856 & 0.920 & 0.988 & 0.906 & 0.972 & 1.043 & 0.907 & 0.973 & 1.045 & 0.914 & 0.981 & 1.053 \\
\hline Lymphocytes-24 & 0.767 & 0.958 & 1.197 & 0.864 & 1.070 & 1.325 & 0.752 & 0.925 & 1.139 & 0.824 & 1.012 & 1.242 \\
\hline Neutrophils-03 & 0.922 & 1.050 & 1.195 & 0.885 & 1.006 & 1.143 & 0.957 & 1.087 & 1.236 & 0.855 & 0.972 & 1.104 \\
\hline Neutrophils -06 & 0.933 & 1.014 & 1.102 & 0.947 & 1.027 & 1.115 & 0.936 & 1.016 & 1.102 & 0.890 & 0.966 & 1.048 \\
\hline Neutrophils-12 & 1.049 & 1.224 & 1.428 & 0.917 & 1.067 & 1.242 & 0.955 & 1.111 & 1.293 & 0.922 & 1.073 & 1.249 \\
\hline Neutrophils -24 & 0.874 & 1.063 & 1.293 & 0.777 & 0.938 & 1.132 & 0.875 & 1.050 & 1.261 & 0.814 & 0.976 & 1.169 \\
\hline Monocytes -03 & 0.781 & 0.918 & 1.079 & 0.795 & 0.932 & 1.092 & 0.894 & 1.048 & 1.229 & 0.893 & 1.047 & 1.228 \\
\hline
\end{tabular}




\begin{tabular}{|c|c|c|c|c|c|c|c|c|c|c|c|c|}
\hline Monocytes -06 & 0.652 & 0.816 & 1.021 & 0.730 & 0.911 & 1.135 & 0.745 & 0.929 & 1.158 & 0.585 & 0.730 & 0.910 \\
\hline Monocytes -12 & 0.857 & 1.108 & 1.433 & 0.956 & 1.232 & 1.587 & 0.899 & 1.159 & 1.492 & 0.990 & 1.276 & 1.643 \\
\hline Monocytes -24 & 0.736 & 1.115 & 1.689 & 0.821 & 1.223 & 1.824 & 0.807 & 1.189 & 1.752 & 0.736 & 1.080 & 1.586 \\
\hline Eosinophils -03 & 0.679 & 0.970 & 1.385 & 0.500 & 0.710 & 1.009 & 0.710 & 1.009 & 1.432 & 0.771 & 1.095 & 1.555 \\
\hline Eosinophils -06 & 0.768 & 0.992 & 1.281 & 0.678 & 0.872 & 1.121 & 0.824 & 1.060 & 1.363 & 0.693 & 0.892 & 1.147 \\
\hline Eosinophils -12 & 0.851 & 1.067 & 1.337 & 0.759 & 0.947 & 1.183 & 0.696 & 0.869 & 1.086 & 0.875 & 1.093 & 1.364 \\
\hline Eosinophils -24 & 0.824 & 1.181 & 1.694 & 1.032 & 1.460 & 2.065 & 0.739 & 1.034 & 1.447 & 0.803 & 1.120 & 1.563 \\
\hline
\end{tabular}

Table 10 Means and coefficient of variation (CV) for study A. Means of GM feeds which are significantly different from the non-GM Control feed are marked, with red background colouring, as follows: $D$ : $P<0.01$ by Dunnett-test, $d: P<0.05$ by Dunnett-test, $T: P<0.01$ by $t$-test but not by Dunnett-test, $t: \mathrm{P}<0.05$ by t-test but not by Dunnett-test, $\mathrm{W}: \mathrm{P}<0.01$ by Wilcoxon signed rank test, $\mathrm{w}: \mathrm{P}<0.05$ by Wilcoxon signed rank test. Dunnett- and t-tests are based on an ANOVA with 5 treatment groups, while Wilcoxon tests only uses data for the specific GM feed and the control feed.

\begin{tabular}{|c|c|c|c|c|c|c|c|c|c|c|c|c|c|c|}
\hline \multirow{2}{*}{$\begin{array}{l}\text { diffWBC } \\
\text { Males } \\
\end{array}$} & \multicolumn{2}{|c|}{ Control } & \multicolumn{3}{|c|}{ NK11- } & \multicolumn{3}{|c|}{ NK33- } & \multicolumn{3}{|c|}{ NK11+ } & \multicolumn{3}{|c|}{ NK33+ } \\
\hline & Mean & $\mathrm{CV}$ & Mean & $\mathrm{CV}$ & Sig & Mean & $\mathrm{CV}$ & Sig & Mean & $\mathrm{CV}$ & Sig & Mean & $\mathrm{CV}$ & Sig \\
\hline Lymphocytes-03 & 71.31 & 5.8 & 70.76 & 8.0 & & 71.40 & 7.3 & & 70.68 & 8.3 & & 70.61 & 12.0 & \\
\hline Lymphocytes-06 & 69.65 & 7.5 & 70.80 & 6.4 & & 68.72 & 7.7 & & 70.05 & 9.3 & & 71.34 & 6.1 & \\
\hline Lymphocytes-12 & 61.49 & 9.8 & 60.50 & 8.8 & & 62.35 & 9.6 & & 61.33 & 12.6 & & 62.03 & 8.9 & \\
\hline Lymphocytes-24 & 47.07 & 31.2 & 45.97 & 23.5 & & 49.61 & 15.6 & & 48.79 & 25.5 & & 46.18 & 32.5 & \\
\hline Neutrophils-03 & 24.71 & 16.9 & 25.15 & 22.6 & & 25.88 & 32.7 & & 25.02 & 23.8 & & 25.44 & 33.0 & \\
\hline Neutrophils -06 & 26.95 & 18.0 & 26.14 & 15.4 & & 27.43 & 19.3 & & 26.33 & 22.0 & & 25.11 & 15.9 & \\
\hline Neutrophils -12 & 36.41 & 15.8 & 37.12 & 14.9 & & 35.05 & 17.2 & & 37.26 & 24.2 & & 35.70 & 15.6 & \\
\hline Neutrophils -24 & 48.24 & 28.2 & 49.72 & 23.0 & & 45.58 & 18.1 & & 46.63 & 27.0 & & 50.00 & 28.8 & \\
\hline Monocytes -03 & 1.887 & 24.1 & 2.200 & 36.0 & & 2.012 & 36.2 & & 2.075 & 40.8 & & 1.988 & 31.7 & \\
\hline Monocytes -06 & 1.200 & 40.9 & 0.899 & 43.7 & tw & 1.100 & 46.9 & & 0.987 & 50.3 & tw & 1.025 & 35.3 & \\
\hline Monocytes -12 & 0.526 & 63.3 & 0.600 & 61.1 & & 0.688 & 62.3 & & 0.702 & 61.3 & & 0.687 & 73.6 & \\
\hline Monocytes -24 & 3.488 & 80.8 & 3.717 & 57.4 & & 3.167 & 53.6 & & 3.163 & 51.5 & & 2.842 & 79.9 & \\
\hline Eosinophils -03 & 2.087 & 47.6 & 1.887 & 56.4 & & 2.187 & 42.9 & & 2.225 & 44.3 & & 1.962 & 53.1 & \\
\hline Eosinophils -06 & 2.200 & 54.5 & 2.161 & 60.4 & & 2.750 & 45.3 & & 2.638 & 62.6 & & 2.525 & 33.5 & \\
\hline Eosinophils -12 & 1.575 & 68.0 & 1.762 & 61.1 & & 1.887 & 39.5 & & 1.798 & 75.6 & & 1.575 & 50.5 & \\
\hline Eosinophils -24 & 1.202 & 78.0 & 1.424 & 71.1 & & 1.643 & 50.8 & & 1.413 & 83.1 & & 0.974 & 100.1 & \\
\hline
\end{tabular}




\begin{tabular}{|c|c|c|c|c|c|c|c|c|c|c|c|c|c|c|}
\hline \multirow{2}{*}{$\begin{array}{l}\text { diffWBC } \\
\text { Females }\end{array}$} & \multicolumn{2}{|c|}{ Control } & \multicolumn{3}{|c|}{ NK11- } & \multicolumn{3}{|c|}{ NK33- } & \multicolumn{3}{|c|}{ NK11+ } & \multicolumn{3}{|c|}{ NK33+ } \\
\hline & Mean & $\mathrm{CV}$ & Mean & $\mathrm{CV}$ & Sig & Mean & $\mathrm{CV}$ & Sig & Mean & $\mathrm{CV}$ & Sig & Mean & $\mathrm{CV}$ & Sig \\
\hline Lymphocytes-03 & 72.86 & 10.3 & 71.88 & 10.0 & & 73.79 & 9.1 & & 71.21 & 9.2 & & 73.23 & 9.6 & \\
\hline Lymphocytes-06 & 67.67 & 6.3 & 67.46 & 6.4 & & 67.40 & 5.8 & & 67.45 & 5.2 & & 69.30 & 6.5 & \\
\hline Lymphocytes-12 & 68.38 & 10.8 & 62.75 & 10.3 & tw & 66.51 & 13.6 & & 66.21 & 10.1 & & 66.70 & 9.9 & \\
\hline Lymphocytes-24 & 49.65 & 28.8 & 46.68 & 24.9 & & 52.92 & 15.2 & & 47.27 & 28.6 & & 49.99 & 26.4 & \\
\hline Neutrophils-03 & 23.20 & 27.7 & 24.44 & 28.9 & & 23.18 & 28.7 & & 24.88 & 24.1 & & 22.57 & 28.5 & \\
\hline Neutrophils -06 & 28.97 & 14.7 & 29.54 & 13.8 & & 29.61 & 14.0 & & 29.26 & 12.9 & & 27.91 & 15.5 & \\
\hline Neutrophils -12 & 28.96 & 25.3 & 34.32 & 18.8 & $d w$ & 30.83 & 28.0 & & 31.31 & 22.2 & & 30.15 & 20.1 & \\
\hline Neutrophils -24 & 46.84 & 29.6 & 49.76 & 22.0 & & 42.89 & 19.9 & & 48.77 & 24.1 & & 46.18 & 29.0 & \\
\hline Monocytes -03 & 1.875 & 37.6 & 1.716 & 41.5 & & 1.725 & 34.2 & & 2.025 & 42.7 & & 1.975 & 36.2 & \\
\hline Monocytes -06 & 1.125 & 53.7 & 0.851 & 75.4 & & 1.012 & 60.7 & & 0.938 & 38.6 & & 0.662 & 59.1 & dTW \\
\hline Monocytes -12 & 0.738 & 128.0 & 0.774 & 72.9 & & 0.943 & 73.1 & & 0.833 & 104.5 & & 0.988 & 91.6 & \\
\hline Monocytes -24 & 2.279 & 94.4 & 2.176 & 66.0 & & 2.375 & 59.8 & & 2.739 & 90.0 & & 2.420 & 66.1 & \\
\hline Eosinophils -03 & 2.063 & 59.0 & 1.964 & 61.8 & & 1.312 & 60.5 & & 1.875 & 42.1 & & 2.200 & 50.5 & \\
\hline Eosinophils -06 & 2.238 & 33.9 & 2.213 & 38.7 & & 1.975 & 49.6 & & 2.337 & 33.0 & & 2.125 & 58.0 & \\
\hline Eosinophils -12 & 1.925 & 36.0 & 2.155 & 57.2 & & 1.886 & 53.6 & & 1.643 & 34.9 & & 2.162 & 33.6 & \\
\hline Eosinophils -24 & 1.235 & 53.8 & 1.382 & 68.0 & & 1.812 & 61.3 & $\mathrm{t}$ & 1.216 & 60.6 & & 1.409 & 60.1 & \\
\hline
\end{tabular}




\subsection{Classical statistical analysis for Study A}

Table 10 presents the results of the t-tests, of Dunnett's tests and of Wilcoxon tests for the diffWBC endpoints for males and females. These result from a classical analysis of variance, according to the randomized block design, which is performed on the cage means after log transforming the data. For ease of interpretation results are expressed as means and coefficients of variation on the original scale, rather than as means and standard deviations on the log scale. Note however that $95 \%$ confidence intervals on the ratio scale are given in Table 9 and in Figure 10 and Figure 11. Exact p-values for the classical tests are given in Appendix 5.

Results of the Shapiro-Wilks test for normality and Bartlett's and Levene's test for homogeneity of variance are given in Appendix 6. Bartlett's' homogeneity test is significant at the $5 \%$ level for males for 3 variables (Lymphocytes-03, Lymphocytes-24 and Neutrophils-24) and for females for 2 variables (Lymphocytes-24 and Eosinophils-06). Levene's homogeneity test is significant for male variable Lymphocytes-03, and for no females variables. This implies that the important assumption of homogeneity of variance is generally fulfilled. For these significant cases, one might resort to Wilcoxon's' test instead of the t-test.

\subsection{Standardized effect sizes for Study A}

SES 95\% confidence intervals are given in Figure 12 and Figure 13. The number of intervals that extend outside the $-/+1$ SD limits equals 23 out of $128(18 \%)$.

\subsection{Equivalence testing for study $\mathrm{C}$ using historical data}

Figure 14 displays the ratio of the residual variance in the current G-TwYST study C as a percentage of the residual variance in the historical GRACE study. This indicates that, except for Eosinophils, the G-TwYST data are much more variable than the Grace data, resulting in a less useful equivalence analysis.

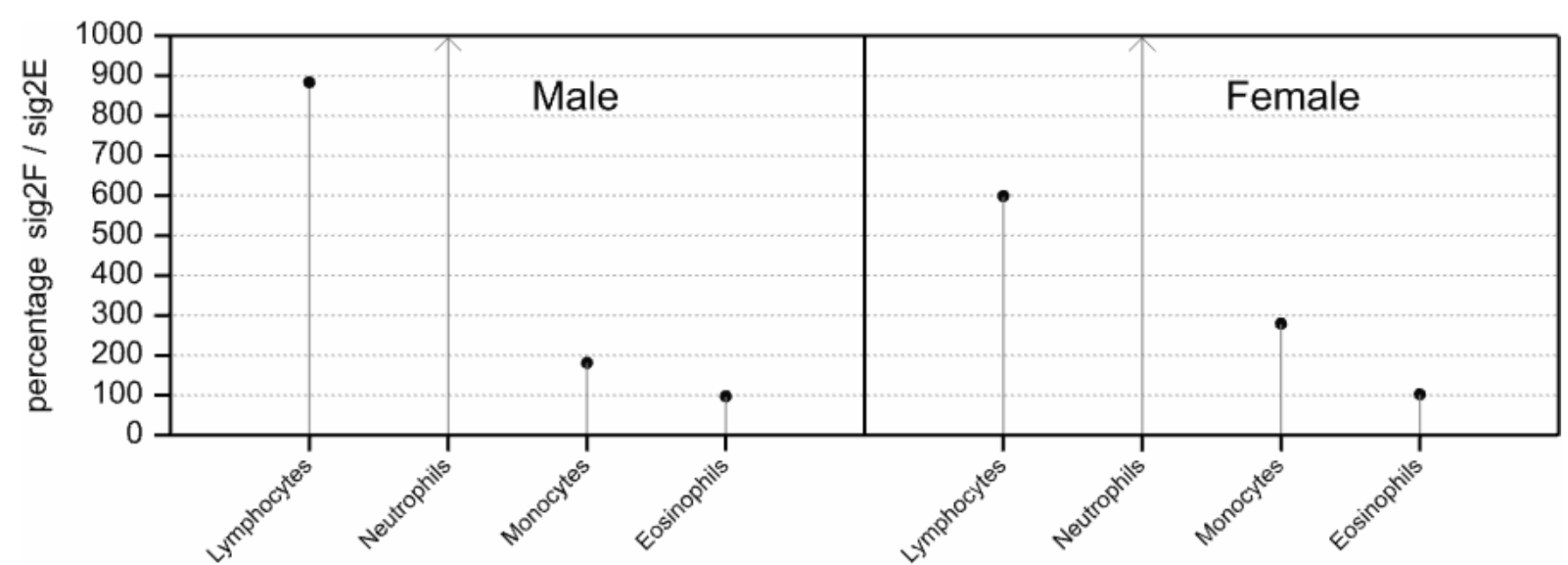

Figure 14 Residual variance (sig2F or $\sigma_{F}^{2}$ ) in the current G-TwYST C study as a percentage of the residual variance (sig2E or $\sigma_{E}^{2}$ ) in the historical GRACE studies.

Preliminary regulatory values $\alpha=0.05, \beta=0.20$ and $n_{0}=8$ were employed, where $n_{0}$ is equal to the number of cages for the diffWBC endpoints (Table 8). The intervals showing the main results of the equivalence tests are given in Figure 15; Figure 16 provides an alternative representation of the same intervals. The hypothesis of no difference is rejected in case the interval does not contain zero, which is denoted by fuchsia coloured estimates. The non-equivalence hypothesis is rejected when 
the interval fully lies inside the interval $(-1,1)$. For further interpretation the $95 \%$ confidence intervals for the ratios are given in Table 11 and Table 12, and also in Figure 17.

Table $1195 \%$ Confidence interval plus estimate for the ratio $\Delta$ of the GMO feeds with $50 \%$ maize inclusion rate versus the corresponding non-GM control feed for study $C$. Ratios with corresponding 95/99\% intervals that do not encompass the value 1 are coloured yellow/gold; this is equivalent to a significant difference according to a $5 / 1 \% \mathrm{t}$-test.

\begin{tabular}{|l|c|c|c|c|c|c|c|c|c|c|c|c|}
\hline diffWBC & \multicolumn{3}{|c|}{ NK11-/50 vs Con50 } & \multicolumn{3}{|c|}{ NK50- vs Con50 } & \multicolumn{3}{c|}{ NK11+/50 vs Con50 } & \multicolumn{3}{|c|}{ NK50+ vs Con50 } \\
\hline Males & low & ratio & upp & low & ratio & upp & low & ratio & upp & low & ratio & upp \\
\hline Lymphocytes & 0.89 & 1.02 & 1.16 & 0.92 & 1.05 & 1.20 & 0.83 & 0.95 & 1.09 & 0.80 & 0.92 & 1.05 \\
\hline Neutrophils & 0.35 & 0.74 & 1.55 & 0.19 & 0.40 & 0.84 & 0.56 & 1.17 & 2.46 & 0.60 & 1.26 & 2.66 \\
\hline Monocytes & 0.53 & 0.82 & 1.27 & 0.48 & 0.75 & 1.17 & 0.58 & 0.90 & 1.40 & 0.64 & 1.00 & 1.55 \\
\hline Eosinophils & 0.68 & 1.08 & 1.71 & 0.63 & 1.01 & 1.60 & 0.70 & 1.12 & 1.78 & 0.76 & 1.21 & 1.93 \\
\hline
\end{tabular}

\begin{tabular}{|l|c|c|c|c|c|c|c|c|c|c|c|c|}
\hline diffWBC & \multicolumn{1}{|c|}{ NK11-/50 vs Con50 } & \multicolumn{3}{|c|}{ NK50- vs Con50 } & \multicolumn{3}{|c|}{ NK11+/50 vs Con50 } & \multicolumn{3}{|c|}{ NK50+ vs Con50 } \\
\hline Females & low & ratio & upp & low & ratio & upp & low & ratio & upp & low & ratio & upp \\
\hline Lymphocytes & 0.91 & 1.03 & 1.15 & 0.94 & 1.06 & 1.19 & 0.89 & 1.00 & 1.12 & 0.92 & 1.04 & 1.16 \\
\hline Neutrophils & 0.46 & 0.81 & 1.40 & 0.48 & 0.83 & 1.44 & 0.56 & 0.98 & 1.70 & 0.42 & 0.73 & 1.26 \\
\hline Monocytes & 0.70 & 1.12 & 1.80 & 0.69 & 1.10 & 1.78 & 0.56 & 0.91 & 1.46 & 0.75 & 1.21 & 1.94 \\
\hline Eosinophils & 0.68 & 1.12 & 1.86 & 0.60 & 0.99 & 1.64 & 0.57 & 0.95 & 1.56 & 0.90 & 1.48 & 2.44 \\
\hline
\end{tabular}

Table $1295 \%$ Confidence interval plus estimate for the ratio $\Delta$ of the feeds with $50 \%$ maize inclusion rate versus the corresponding $33 \%$ inclusion rate for study $\mathrm{C}$, and for the $\mathrm{GM}$ $33 \%$ feeds versus the non-GM $33 \%$ feed. Ratios with corresponding $95 / 99 \%$ intervals that do not encompass the value 1 are coloured yellow/gold; this is equivalent to a significant difference according to a $5 / 1 \%$ t-test.

\begin{tabular}{|l|c|c|c|c|c|c|c|c|c|c|c|c|c|c|c|}
\hline diffWBC & \multicolumn{3}{|c|}{ Con50 vs Con33 } & \multicolumn{2}{c|}{ NK50- vs NK33- } & \multicolumn{3}{c|}{ NK50+ vs NK33+ } & \multicolumn{3}{c|}{ NK33- vs Con33 } & \multicolumn{2}{c|}{ NK33+ vs Con33 } \\
\hline Males & low & ratio & upp & low & ratio & upp & low & ratio & upp & low & ratio & upp & low & ratio & upp \\
\hline Lymphocytes & 1.04 & 1.19 & 1.36 & 0.97 & 1.11 & 1.27 & 0.89 & 1.02 & 1.16 & 0.98 & 1.12 & 1.28 & 0.94 & 1.07 & 1.22 \\
\hline Neutrophils & 0.29 & 0.60 & 1.27 & 0.16 & 0.33 & 0.70 & 0.52 & 1.08 & 2.28 & 0.34 & 0.72 & 1.52 & 0.33 & 0.70 & 1.48 \\
\hline Monocytes & 0.81 & 1.26 & 1.96 & 0.44 & 0.68 & 1.05 & 0.75 & 1.16 & 1.81 & 0.90 & 1.40 & 2.17 & 0.70 & 1.08 & 1.68 \\
\hline Eosinophils & 0.51 & 0.81 & 1.29 & 0.48 & 0.76 & 1.20 & 0.56 & 0.90 & 1.43 & 0.68 & 1.08 & 1.72 & 0.69 & 1.09 & 1.74 \\
\hline
\end{tabular}

\begin{tabular}{|l|l|l|l|l|l|l|l|l|l|l|l|l|l|l|l|l|}
\hline diffWBC & \multicolumn{3}{|c|}{ Con50 vs Con33 } & \multicolumn{2}{c|}{ NK50- vs NK33- } & \multicolumn{3}{c|}{ NK50+ vs NK33+ } & \multicolumn{2}{c|}{ NK33- vs Con33 } & \multicolumn{2}{c|}{ NK33+ vs Con33 } \\
\hline Females & low & ratio & upp & low & ratio & upp & low & ratio & upp & low & ratio & upp & low & ratio & upp \\
\hline Lymphocytes & 0.80 & 0.90 & 1.00 & 0.92 & 1.04 & 1.16 & 0.91 & 1.03 & 1.15 & 0.82 & 0.91 & 1.03 & 0.81 & 0.90 & 1.01 \\
\hline Neutrophils & 0.95 & 1.65 & 2.88 & 0.52 & 0.91 & 1.58 & 0.47 & 0.82 & 1.42 & 0.87 & 1.51 & 2.62 & 0.84 & 1.47 & 2.55 \\
\hline Monocytes & 0.75 & 1.21 & 1.94 & 0.59 & 0.95 & 1.53 & 0.81 & 1.31 & 2.11 & 0.87 & 1.40 & 2.25 & 0.69 & 1.11 & 1.79 \\
\hline Eosinophils & 0.65 & 1.07 & 1.76 & 0.58 & 0.95 & 1.57 & 0.88 & 1.46 & 2.40 & 0.68 & 1.12 & 1.84 & 0.66 & 1.08 & 1.79 \\
\hline
\end{tabular}




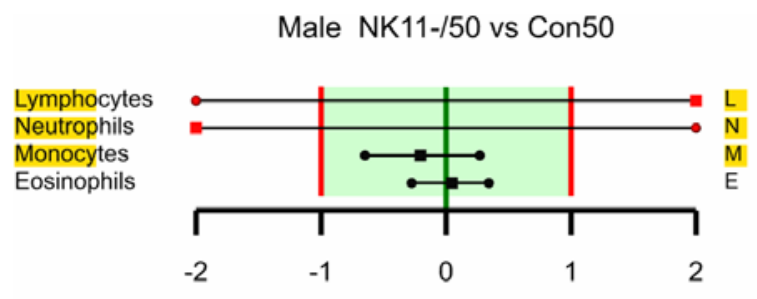

Female NK11-/50 vs Con50

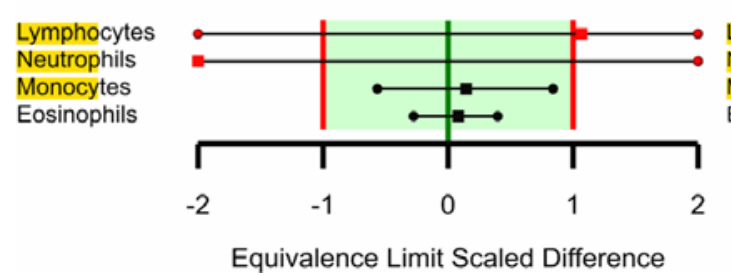

Male NK50- vs Con50

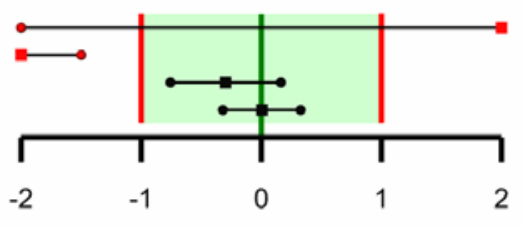

Female NK50-vs Con50

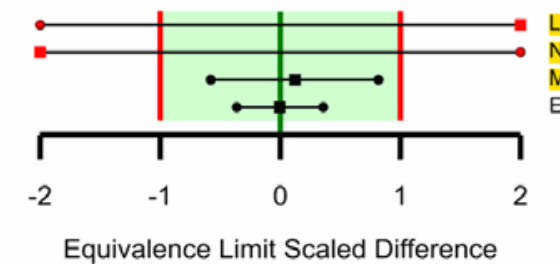

Male NK $11+/ 50$ vs Con50

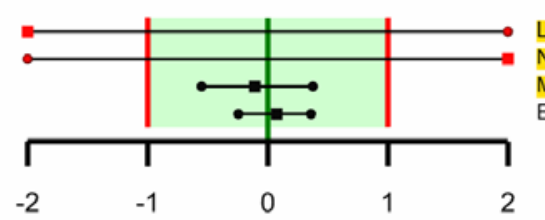

Female NK11+/50 vs Con50

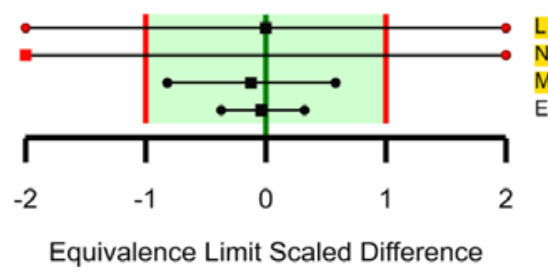

Male NK50+ vs Con50

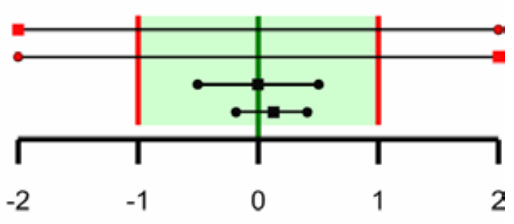

Female NK50+ vs Con50

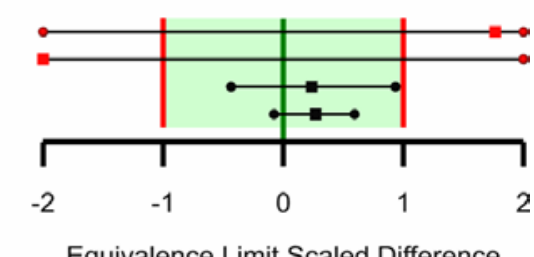

Male Con50 vs Con33

Male NK50- vs NK33-

Male NK50+ vs NK33+

Male NK33- vs Con33

Male NK33+ vs Con33

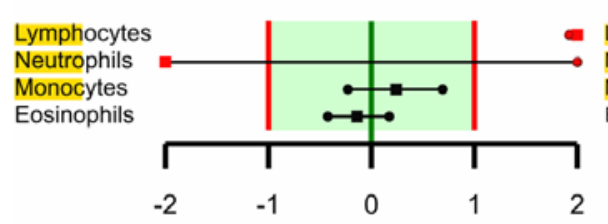

Female Con50 vs Con33
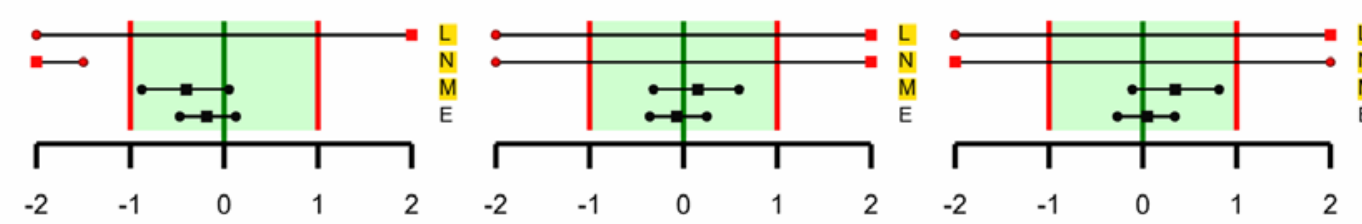

Female NK50- vs NK33-

Female NK50+ vs NK33+

Female NK33-vs Con33

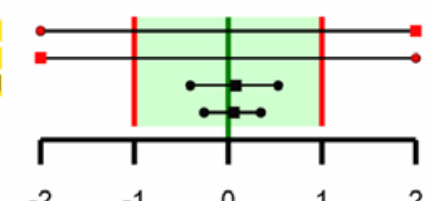

Female NK33+vs Con33
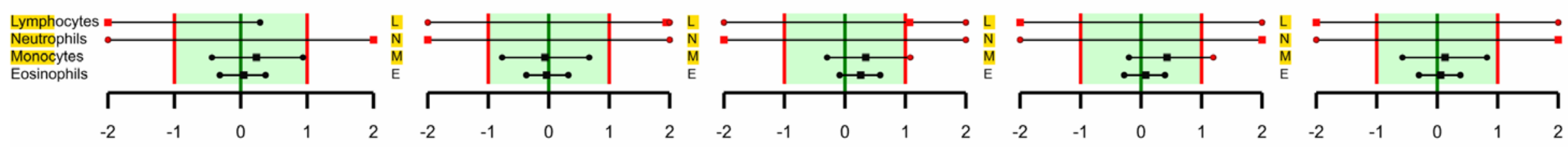

Equivalence Limit Scaled Difference Equivalence Limit Scaled Difference Equivalence Limit Scaled Difference Equivalence Limit Scaled Difference

Figure 15 Equivalence testing of GM feeding groups versus the control feed for males in study C. Endpoints labelled with a golden background have a large residual variance compared to the historical studies (VR>150\%). Fuchsia coloured symbols denote a significant difference. 

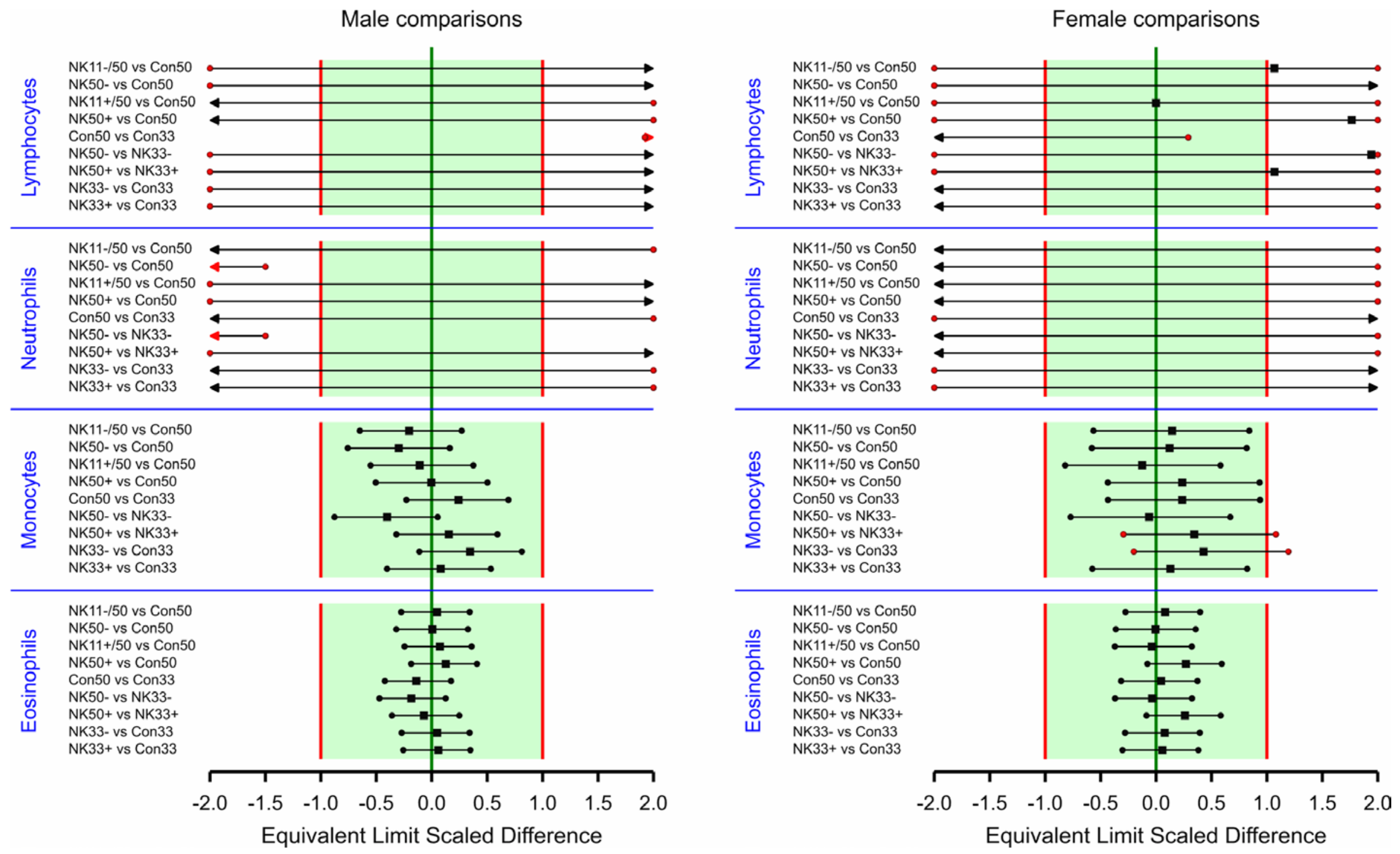

Figure 16 Equivalence testing of GM feeding groups versus the control feed for males in study C; alternative representation of the intervals in Figure 15. 


\section{Male Comparisons}

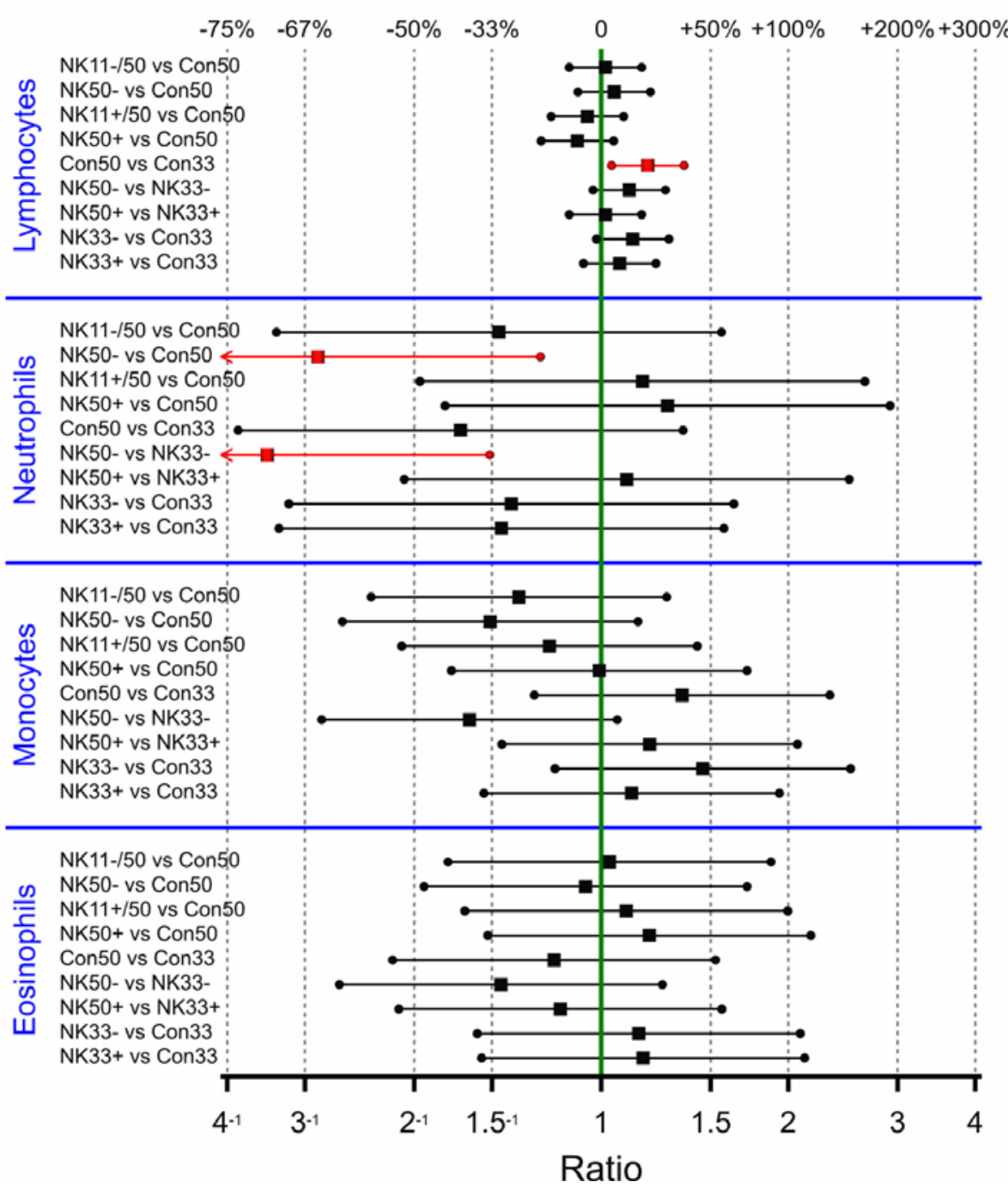

\section{Female Comparisons}

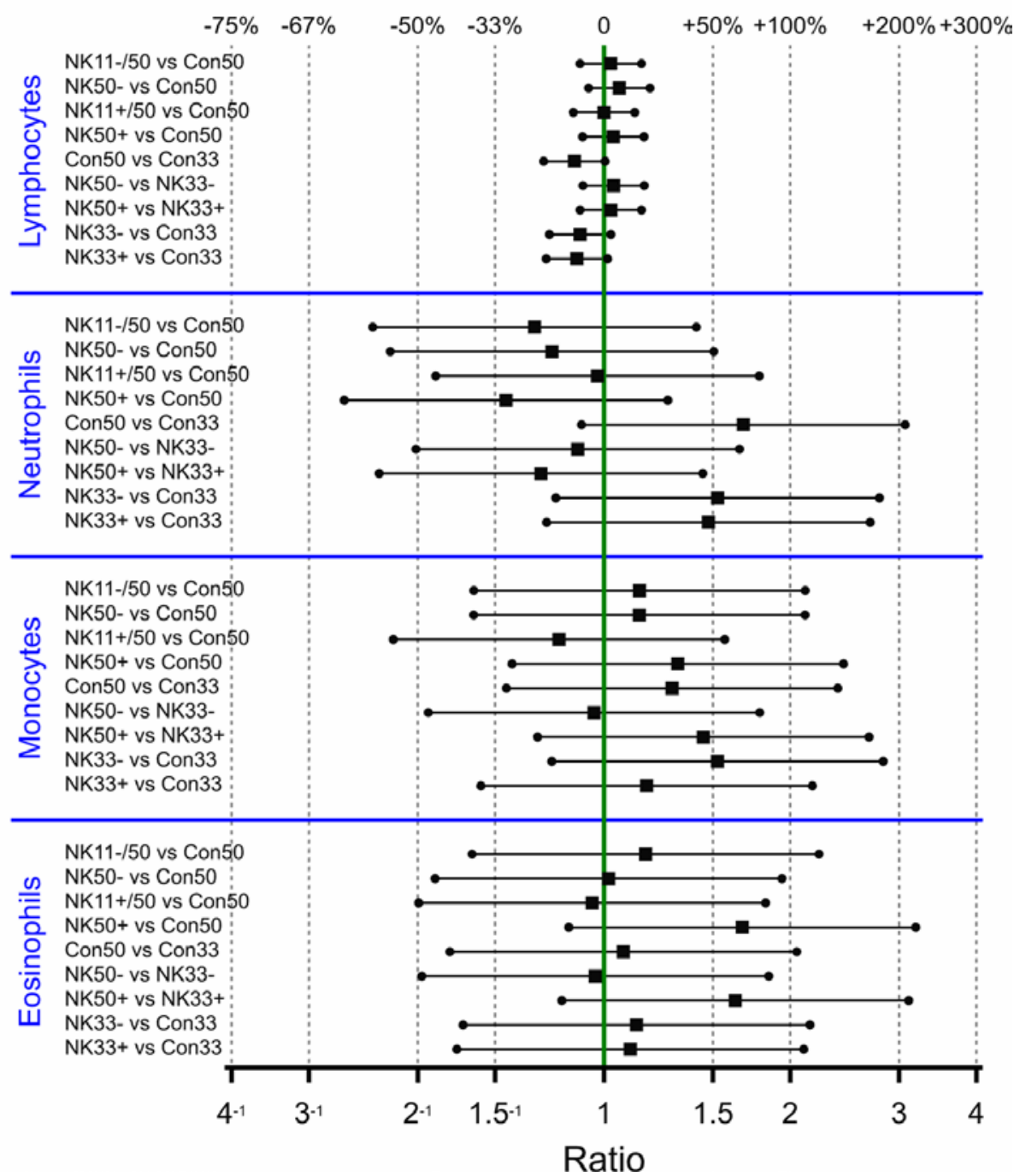

Figure $1795 \%$ confidence intervals for the nine comparisons in Study $C$. Intervals that do not encompass the ratio=1 line are given in red, these represent significant differences at the $5 \%$ level. 


\subsection{Classical statistical analysis for Study C}

Table 13 presents the results of the t-tests, of Dunnett's tests and of Wilcoxon tests for the study $C$ diffWBC endpoints for males and females. These result from a classical analysis of variance, according to the randomized block design, which is performed on the cage means after log transforming the data. For ease of interpretation results are expressed as means on the original scale, rather than on the log scale. Note however that $95 \%$ confidence intervals on the ratio scale are given in Table 11 and Table 12, and also in Figure 17. Exact p-values for the classical tests are given in Appendix 5

Results of the Shapiro-Wilks test for normality and Bartlett's and Levene's test for homogeneity of variance are given in Appendix 6. Bartlett's' homogeneity test is significant at the $5 \%$ level for male variable Neutrophils and for females variables Lymphocytes and Neutrophils. Levene's homogeneity test is significant for male variables Neutrophils and Monocytes, and for no female variables. For these significant cases, one might resort to Wilcoxon's' test instead of the t-test.

\subsection{Standardized effect sizes for Study C}

SES 95\% confidence intervals are given in Figure 18. The SES interval lies completely within the $(-1,1)$ interval only when the difference between the group means is very close to zero. 
Table 13 Means per feeding group and results of statistical tests for nine comparisons for Study $C$. Significant difference are marked, with red background colouring, as follows: $D$ : $P<0.01$ by Dunnett-test, $d: P<0.05$ by Dunnett-test, $T: P<0.01$ by t-test but not by Dunnett-test, $t: P<0.05$ by $t$-test but not by Dunnett-test, $W: P<0.01$ by Wilcoxon signed rank test, $w$ : $P<0.05$ by Wilcoxon signed rank test. See text.

\begin{tabular}{|c|c|c|c|c|c|c|c|c|c|c|c|c|c|c|c|c|c|}
\hline diffWBC & \multicolumn{8}{|c|}{ Means } & NK11- & NK50- & NK11+ & NK50+ & Con50 & NK50- & NK50+ & NK33- & NK33+ \\
\hline Males & Con50 & NK11- & NK50- & NK11+ & NK50+ & Con33 & NK33- & NK33+ & Con50 & Con50 & Con50 & Con50 & Con33 & NK33- & NK33+ & Con33 & Con33 \\
\hline Lymphocytes & 78.09 & 79.41 & 82.66 & 74.41 & 73.78 & 67.00 & 74.09 & 71.94 & & & & & tW & & & & \\
\hline Neutrophils & 18.75 & 17.06 & 14.25 & 22.00 & 22.38 & 29.19 & 21.28 & 24.41 & & $\mathrm{t}$ & & & & $\mathrm{T}$ & & & \\
\hline Monocytes & 1.969 & 2.031 & 1.625 & 2.094 & 2.000 & 1.625 & 2.781 & 1.781 & & & & & & & & & \\
\hline Eosinophils & 1.156 & 1.469 & 1.469 & 1.500 & 1.844 & 2.188 & 1.844 & 1.875 & & & & & & & & & \\
\hline
\end{tabular}

\begin{tabular}{|c|c|c|c|c|c|c|c|c|c|c|c|c|c|c|c|c|c|}
\hline diffWBC & \multicolumn{8}{|c|}{ Means } & \multirow{2}{*}{$\begin{array}{l}\text { NK11- } \\
\text { Con50 }\end{array}$} & \multirow{2}{*}{$\begin{array}{l}\text { NK50- } \\
\text { Con50 }\end{array}$} & \multirow{2}{*}{$\begin{array}{l}\text { NK11+ } \\
\text { Con50 }\end{array}$} & \multirow{2}{*}{$\begin{array}{l}\text { NK50+ } \\
\text { Con50 }\end{array}$} & \multirow{2}{*}{$\begin{array}{l}\text { Con50 } \\
\text { Con33 }\end{array}$} & \multirow{2}{*}{$\begin{array}{l}\text { NK50- } \\
\text { NK33- }\end{array}$} & \multirow{2}{*}{$\begin{array}{l}\text { NK50+ } \\
\text { NK33+ }\end{array}$} & \multirow{2}{*}{$\begin{array}{l}\text { NK33- } \\
\text { Con33 }\end{array}$} & \multirow{2}{*}{$\begin{array}{l}\text { NK33- } \\
\text { Con3 }\end{array}$} \\
\hline Females & Con50 & NK11- & NK50- & NK11+ & NK50+ & Con33 & NK33- & NK33+ & & & & & & & & & \\
\hline Lymphocytes & 72.72 & 74.03 & 76.41 & 72.44 & 74.84 & 80.53 & 73.84 & 73.16 & & & & & & & & & \\
\hline Neutrophils & 24.75 & 23.03 & 21.00 & 25.28 & 21.88 & 17.44 & 23.12 & 24.44 & & & & & & & & & \\
\hline Monocytes & 1.156 & 1.406 & 1.312 & 1.094 & 1.344 & 0.875 & 1.531 & 0.969 & & & & & & & & & \\
\hline Eosinophils & 1.375 & 1.531 & 1.250 & 1.188 & 1.938 & 1.156 & 1.500 & 1.438 & & & & & & & & & \\
\hline
\end{tabular}



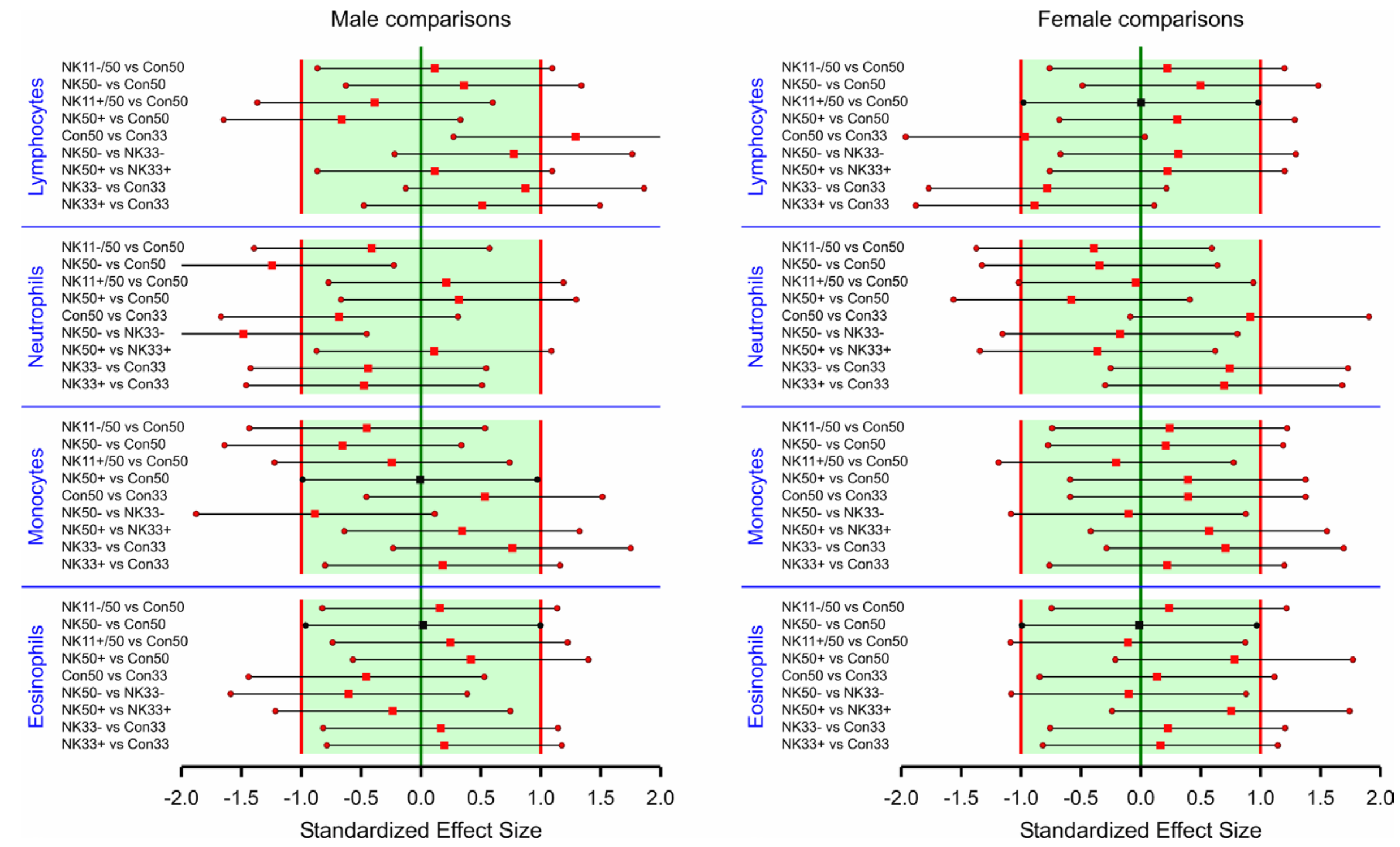

Figure 18 Confidence intervals for Standardized Effect Sized (SES) for Study C. Intervals that extend beyond the interval $(-1,1)$ are given in red. 


\section{References}

Goedhart PW, van der Voet H (2017). G-TwYST Study B. A 90-day toxicity study in rats fed GM maize NK603. Statistical report. Report 31.10.17, Biometris, Wageningen, The Netherlands.

https://doi.org/10.18174/455203 with appendices https://doi.org/10.18174/455204

Goedhart PW, van der Voet H (2018a). G-TwYST Study A. Combined chronic toxicity and carcinogenicity study in rats fed GM maize NK603. Main statistical report. Report 32.02.18, Biometris, Wageningen, The Netherlands. https://doi.org/10.18174/455205

Goedhart PW, van der Voet H (2018b). G-TwYST Study A. Combined chronic toxicity and carcinogenicity study in rats fed GM maize NK603. Statistical report, 3 months. Report 33.02.18, Biometris, Wageningen, The Netherlands. https://doi.org/10.18174/455206

Goedhart PW, van der Voet H (2018c). G-TwYST Study A. Combined chronic toxicity and carcinogenicity study in rats fed GM maize NK603. Statistical report, 6 months. Report 34.02.18, Biometris, Wageningen, The Netherlands. https://doi.org/10.18174/455207

Goedhart PW, van der Voet H (2018d). G-TwYST Study A. Combined chronic toxicity and carcinogenicity study in rats fed GM maize NK603. Statistical report, 12 months. Report 35.02.18, Biometris, Wageningen, The Netherlands. https://doi.org/10.18174/455208

Goedhart PW, van der Voet H (2018e). G-TwYST Study A. Combined chronic toxicity and carcinogenicity study in rats fed GM maize NK603. Statistical report, 24 months. Report 36.02.18, Biometris, Wageningen, The Netherlands. https://doi.org/10.18174/455209

Goedhart PW, van der Voet H (2018f). G-TwYST Study C. A 90-day toxicity study in rats fed GM maize NK603. Statistical report. Report 37.03.18, Biometris, Wageningen, The Netherlands. https://doi.org/10.18174/455210 with appendices https://doi.org/10.18174/455211 


\section{List of Tables}

Table 1 Data files for G-TwYST studies A and C

Table 2 Number of animals for which diffWBC data are available

Table 3 The five smallest and five largest observed diffWBC percentages for every response variable observed in study A for Males. Outliers are listed in yellow.Table 3 The five smallest and five largest observed diffWBC percentages for every response variable observed in study A for Males. Outliers are listed in yellow.

Table 4 The five smallest and five largest observed diffWBC percentages for every response variable observed in study A for Females. Outliers are listed in yellow.

Table 5 The five smallest and five largest observed diffWBC percentages for every response variable observed in study $\mathrm{C}$ for Males. No outliers were identified.

Table 6 The five smallest and five largest observed diffWBC percentages for every response variable observed in study $C$ for Females. No outliers were identified.

Table 7 Summary statistics for male and female rats for Study A classified by the feeding groups: number of cages $(\mathrm{N})$, means (Mean), standard deviations $(\mathrm{Sd})$ and coefficients of variation (CV). The summary statistics are obtained from cage means.Table 7 Summary statistics for male and female rats for Study A classified by the feeding groups: number of cages (N), means (Mean), standard deviations (Sd) and coefficients of variation (CV). The summary statistics are obtained from cage means.Table $8 \quad$ Summary statistics for male and female rats for Study $\mathrm{C}$ classified by the feeding groups: means (Mean), standard deviations (Sd) and coefficients of variation (CV). The summary statistics are obtained from cage means. All means are based on 8 cages.

Table $995 \%$ Confidence interval plus estimate for the ratio $\Delta$ of the GMO feeds versus the Control feed for Study A. Intervals are based on an ANOVA with 5 feeding groups. Ratios with corresponding 95\% confidence intervals that do not encompass the value 1 are coloured red; this is equivalent to a significant difference according to a t-test with significance level $5 \%$.

Table 10 Means and coefficient of variation (CV) for study A. Means of GM feeds which are significantly different from the non-GM Control feed are marked, with red background colouring, as follows: $D: P<0.01$ by Dunnett-test, $d: P<0.05$ by Dunnett-test, $T: P<0.01$ by ttest but not by Dunnett-test, $t: P<0.05$ by t-test but not by Dunnett-test, $W: P<0.01$ by Wilcoxon signed rank test, $\mathrm{w}: \mathrm{P}<0.05$ by Wilcoxon signed rank test. Dunnett- and t-tests are based on an ANOVA with 5 treatment groups, while Wilcoxon tests only uses data for the specific GM feed and the control feed.

Table $1195 \%$ Confidence interval plus estimate for the ratio $\Delta$ of the GMO feeds with $50 \%$ maize inclusion rate versus the corresponding non-GM control feed for study C. Ratios with corresponding 95/99\% intervals that do not encompass the value 1 are coloured yellow/gold; this is equivalent to a significant difference according to a $5 / 1 \%$ t-test. 
Table $1295 \%$ Confidence interval plus estimate for the ratio $\Delta$ of the feeds with $50 \%$ maize inclusion rate versus the corresponding $33 \%$ inclusion rate for study $\mathrm{C}$, and for the GM $33 \%$ feeds versus the non-GM $33 \%$ feed. Ratios with corresponding $95 / 99 \%$ intervals that do not encompass the value 1 are coloured yellow/gold; this is equivalent to a significant difference according to a 5/1\% t-test.

Table 13 Means per feeding group and results of statistical tests for nine comparisons for Study C. Significant difference are marked, with red background colouring, as follows: $D: P<0.01$ by Dunnett-test, $d: \mathrm{P}<0.05$ by Dunnett-test, $T: \mathrm{P}<0.01$ by t-test but not by Dunnett-test, $t: \mathrm{P}<0.05$ by $\mathrm{t}$-test but not by Dunnett-test, $W: \mathrm{P}<0.01$ by Wilcoxon signed rank test, $w$ : $\mathrm{P}<0.05$ by Wilcoxon signed rank test. See text. 


\section{List of Figures}

Figure 1 Residuals along the $y$-axis versus fitted values along the $x$-axis resulting from an analysis of variance for Study A in Males on log transformed cage means.Error! Reference source not found.

Figure 2 Normal probability plots of residuals resulting from an analysis of variance for Study A in Males on log transformed cage means.

Figure 3 Residuals along the $y$-axis versus fitted values along the $x$-axis resulting from an analysis of variance for Study A in Females on log transformed cage means.

Figure 4 Normal probability plots of residuals resulting from an analysis of variance for Study A in Females on log transformed cage means.

Figure 5 Residuals along the $y$-axis versus fitted values along the $x$-axis resulting from an analysis of variance for Study $C$ on log transformed cage means and normal probability plots for males (top) and females (bottom).

Figure 6 Mean values of Lymphocytes and Neutrophils versus month for each feeding group in Study A for males (left) and females (right).

Figure 7 Residual variance (sig2F or $\sigma_{F}^{2}$ ) in the current G-TwYST A study as a percentage of the residual variance (sig2E or $\sigma_{E}^{2}$ ) in the historical GRACE studies for males (top panel) and females (bottom panel) that survived for 24 months.

Figure 8 Equivalence testing of GM feeding groups versus the control feed for males in study A. For estimates (square symbols) on the left of zero the GM feed has a smaller mean than the control feed (see also Table 9). Endpoints labelled with a golden background have a large residual variance compared to the historical studies (variance ratio $V R>150 \%$ ). Fuchsia coloured symbols denote a significant difference.

Figure 9 Equivalence testing of GM feeding groups versus the control feed for females in study $A$. For estimates (square symbols) on the left of zero the GM feed has a smaller mean than the control feed (see also Table 9). Endpoints labelled with a golden background have a large residual variance compared to the historical studies (variance ratio $V R>150 \%$ ). Fuchsia coloured symbols denote a significant difference.

Figure $1095 \%$ confidence intervals for the four comparisons for males in Study A. Intervals that do not encompass the ratio $=1$ line are given in red, these represent significant differences at the $5 \%$ level.

Figure $1195 \%$ confidence intervals for the four comparisons for females in Study A. Intervals that do not encompass the ratio $=1$ line are given in red, these represent significant differences at the $5 \%$ level.

Figure 12 Confidence intervals for Standardized Effect Sized (SES) for male rats in Study A. Intervals that extend beyond the interval $(-1,1)$ are given in red.Figure 12 Confidence intervals for Standardized Effect Sized (SES) for male rats in Study A. Intervals that extend beyond the interval $(-1,1)$ are given in red. 
Figure 13 Confidence intervals for Standardized Effect Sized (SES) for female rats in Study A. Intervals that extend beyond the interval $(-1,1)$ are given in red.

Figure 14 Residual variance (sig2F or $\boldsymbol{\sigma}_{F}^{2}$ ) in the current G-TwYST C study as a percentage of the residual variance (sig2E or $\sigma_{E}^{2}$ ) in the historical GRACE studies.

Figure 15 Equivalence testing of GM feeding groups versus the control feed for males in study C. Endpoints labelled with a golden background have a large residual variance compared to the historical studies (VR>150\%). Fuchsia coloured symbols denote a significant difference.

Figure 16 Equivalence testing of GM feeding groups versus the control feed for males in study C; alternative representation of the intervals in Figure 15.

Figure 17 95\% confidence intervals for the nine comparisons in Study C. Intervals that do not encompass the ratio $=1$ line are given in red, these represent significant differences at the $5 \%$ level.

Figure 18 Confidence intervals for Standardized Effect Sized (SES) for Study C. Intervals that extend beyond the interval $(-1,1)$ are given in red.Figure 18 Confidence intervals for Standardized Effect Sized (SES) for Study C. Intervals that extend beyond the interval $(-1,1)$ are given in red. 


\section{Appendix 1. Graphs of cage means on the original scale for study A}

Red symbols denote means for feeding groups while the red line denotes the overall mean. Note that observations might overlap.

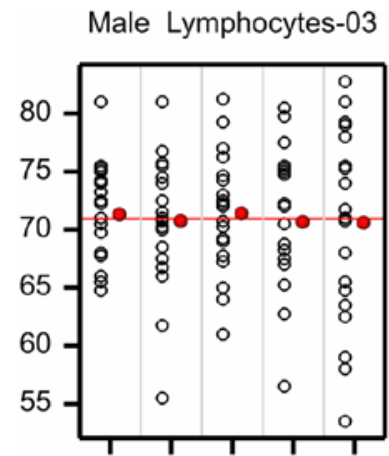

Con 11- 33- 11+ 33+

Male Neutrophils-03

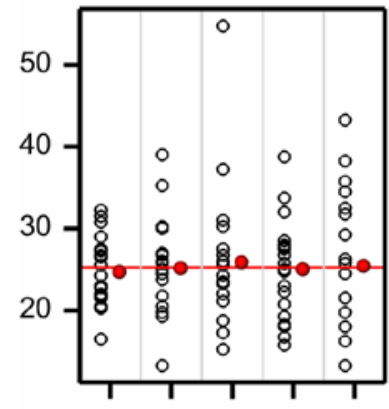

Con 11- 33- 11+ 33+

Male Monocytes-03

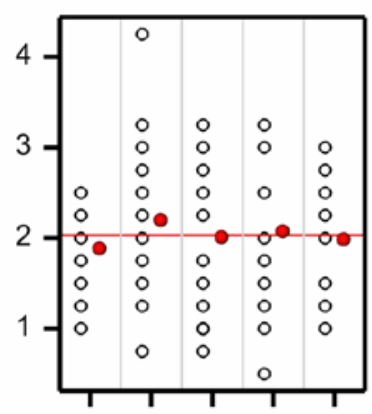

Con 11- 33- 11+ 33+

Male Eosinophils-03

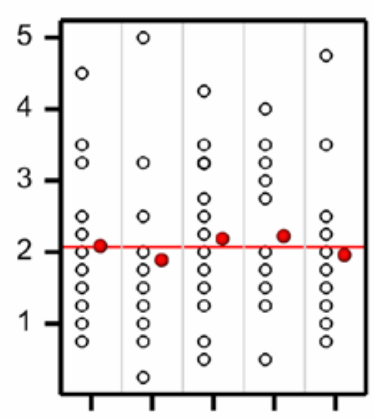

Con 11- 33- 11+ 33+
Male Lymphocytes-06

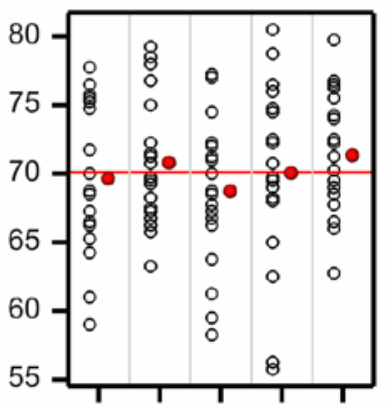

Con 11- 33- 11+33+

Male Neutrophils-06

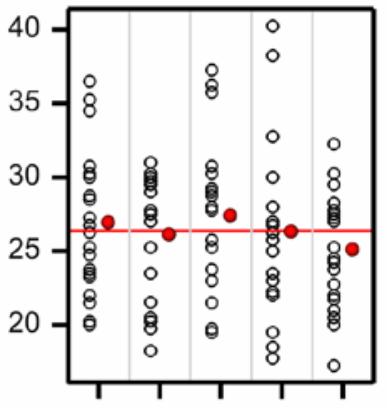

Con 11- 33- 11+ 33+

Male Monocytes-06

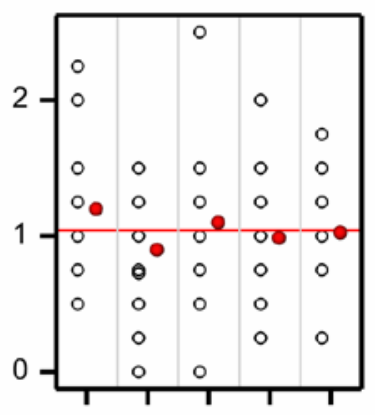

Con 11- 33- 11+ 33+

Male Eosinophils-06

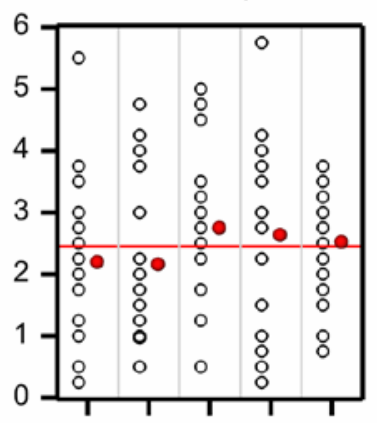

Con 11- 33- 11+ 33+
Male Lymphocytes-12

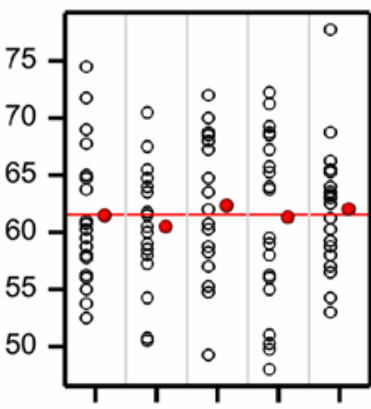

Con 11- 33- 11+ 33+

Male Neutrophils-12

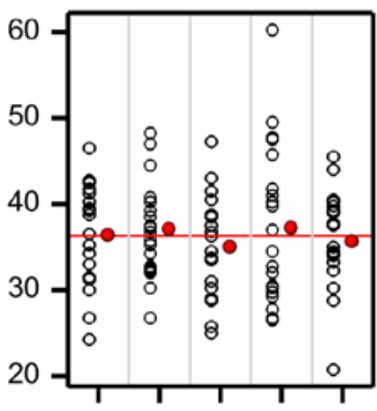

Con 11- 33- $11+33+$

Male Monocytes-12

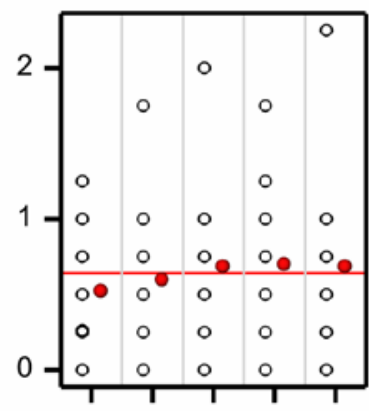

Con 11- 33- 11+ 33+

Male Eosinophils-12

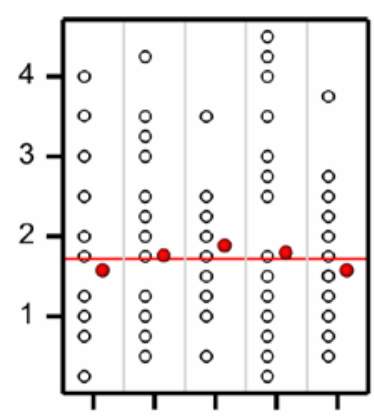

Con 11- 33- 11+ 33+
Male Lymphocytes-24

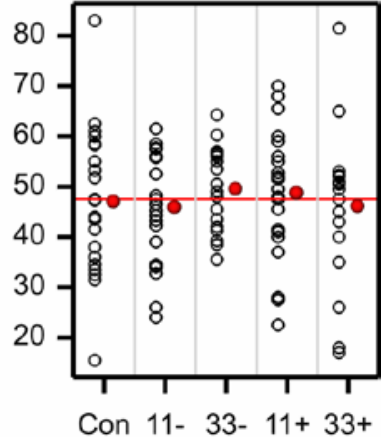

Male Neutrophils-24

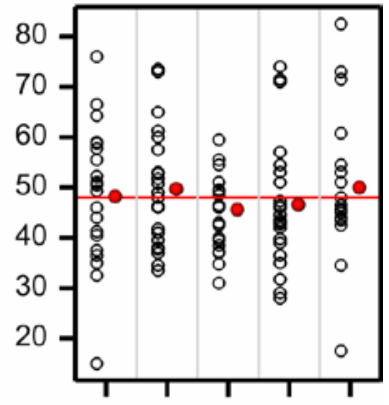

Con 11- 33- $11+33+$

Male Monocytes-24

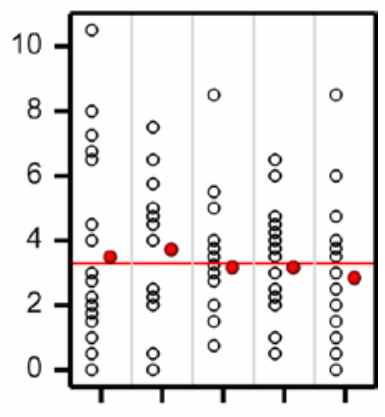

Con 11- 33- 11+33+

Male Eosinophils-24

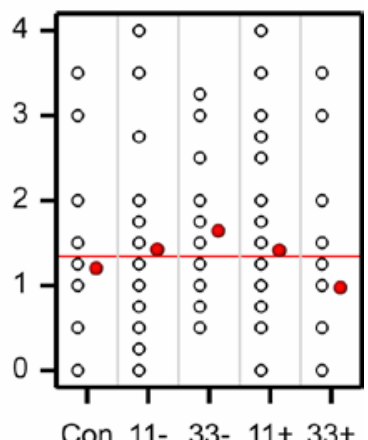




\section{Appendix 1. Graphs of cage means on the original scale for study A( continued)}

Red symbols denote means for feeding groups while the red line denotes the overall mean. Note that observations might overlap.

Female Lymphocytes-03

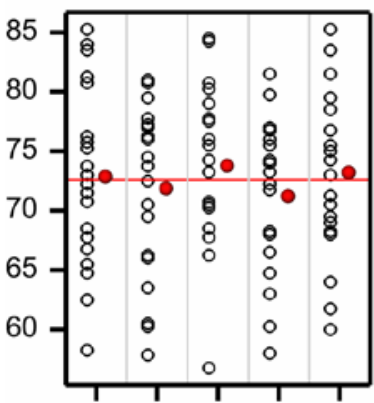

Con 11- 33- $11+33+$

Female Neutrophils-03

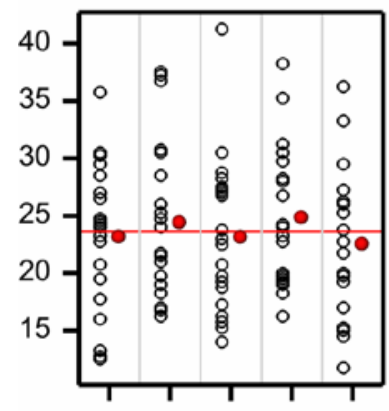

Con 11- 33- 11+33+

Female Monocytes-03

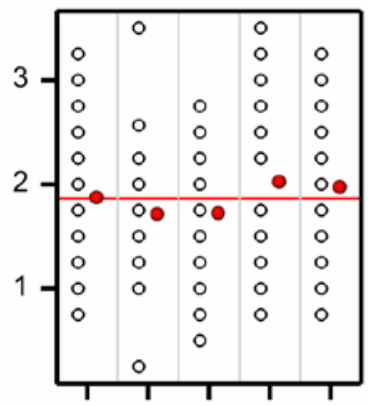

Con 11- 33- 11+ 33+

Female Eosinophils-03

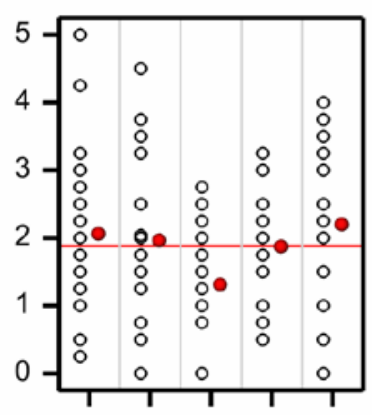

Con 11- 33- 11+ 33+
Female Lymphocytes-06

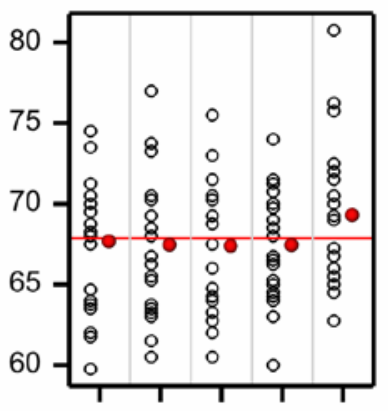

Con 11- 33- 11+ 33+

Female Neutrophils-06

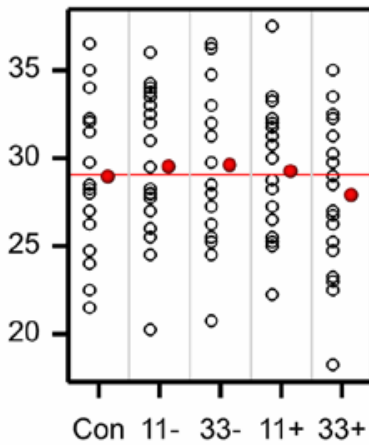

Female Monocytes-06

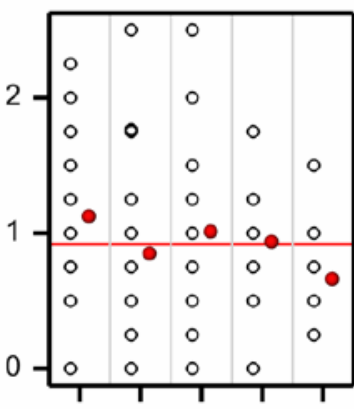

Con 11- 33- $11+33+$

Female Eosinophils-06

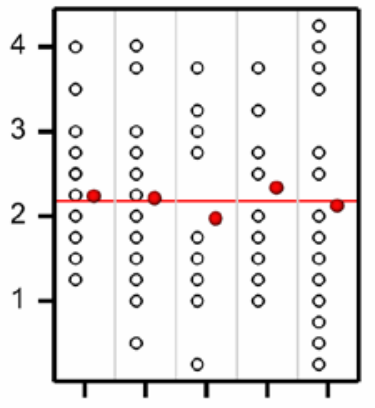

Con 11- 33- $11+33+$
Female Lymphocytes-12

Female Lymphocytes-24

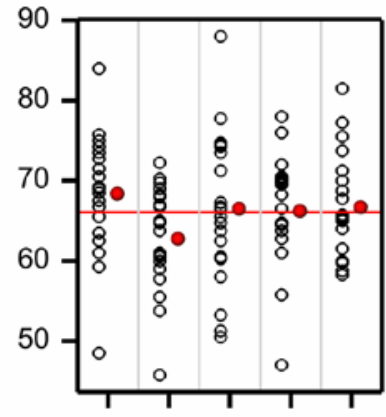

Con 11- 33- 11+ 33+

Female Neutrophils-12

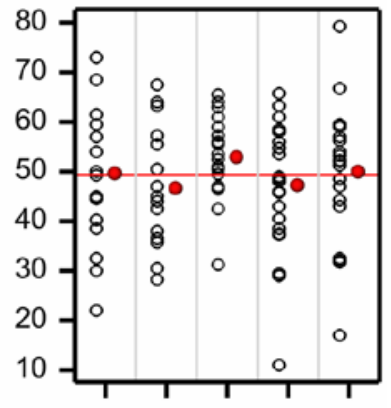

Con 11- 33- 11+ 33+

Female Neutrophils-24

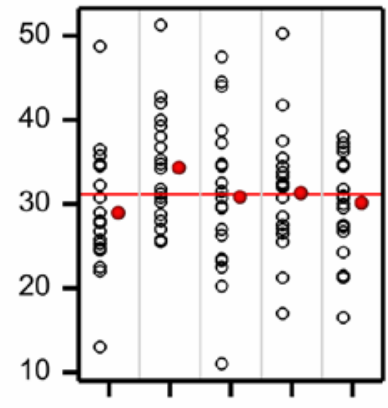

Con 11- 33- 11+33+

Female Monocytes-12

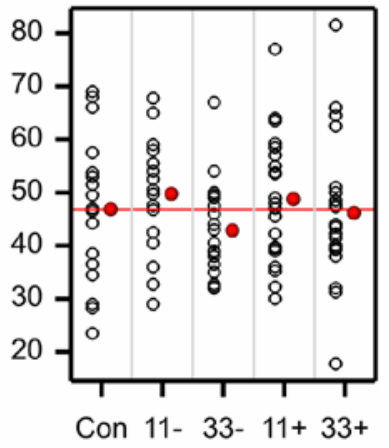

Female Monocytes-24

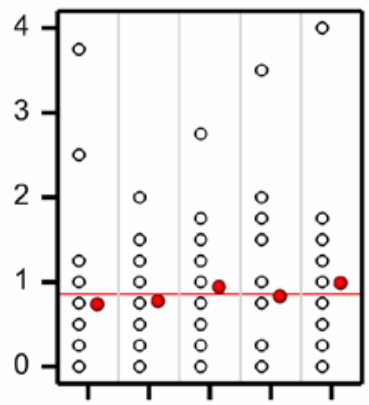

Con 11- 33- 11+ 33+

Female Eosinophils-12

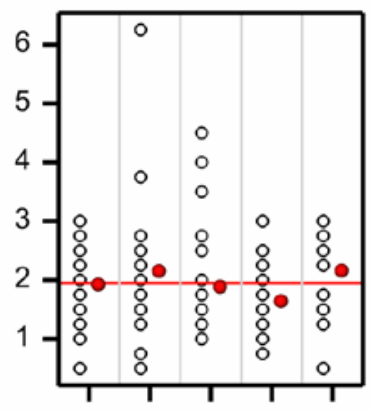

Con 11- 33- 11+ 33+

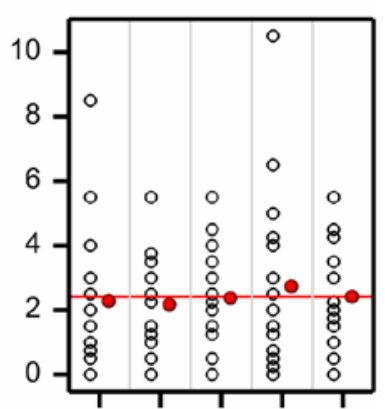

Con 11- 33- 11+ 33+

Female Eosinophils-24

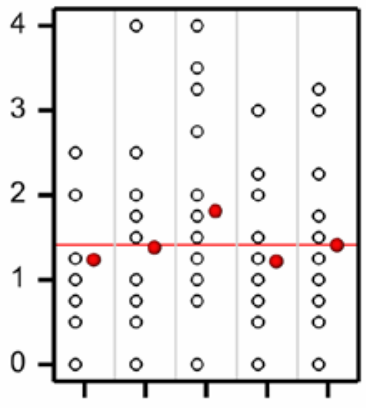

Con 11- 33- 11+ 33+ 


\section{Appendix 2. Graphs of cage means on the log scale for study A}

Red symbols denote means for feeding groups while the red line denotes the overall mean. Note that observations might overlap.

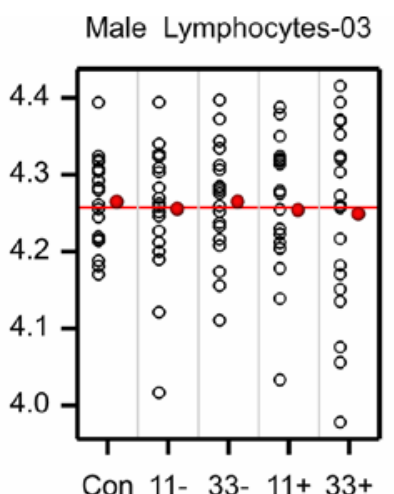

Male Neutrophils-03

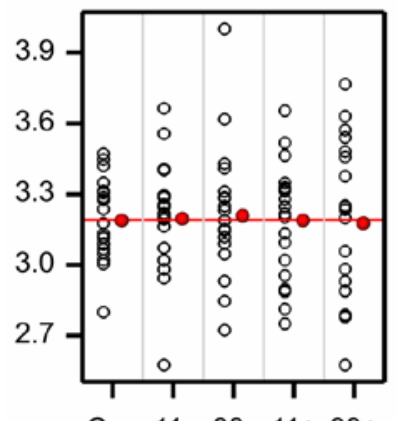

Con 11- 33- 11+ 33+

Male Monocytes-03

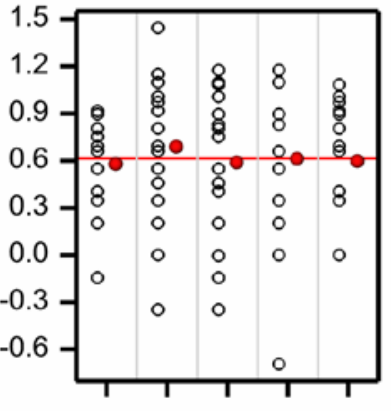

Con 11- 33- 11+ 33+

Male Eosinophils-03

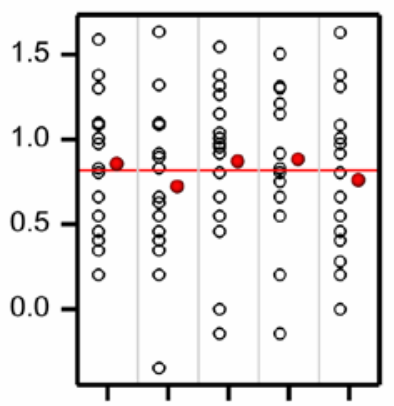

Con 11- 33- 11+ 33+
Male Lymphocytes-06

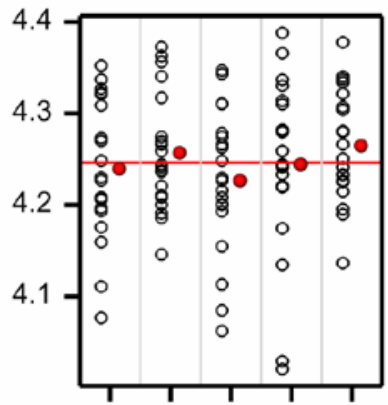

Con 11- 33- $11+33+$

Male Neutrophils-06

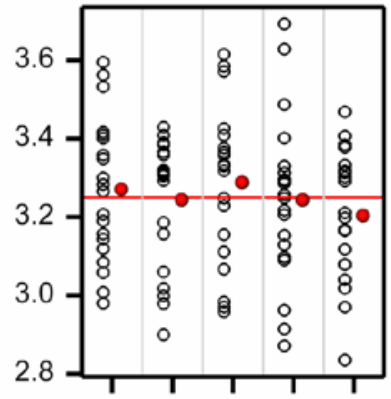

Con 11- 33- $11+33+$

Male Monocytes-06

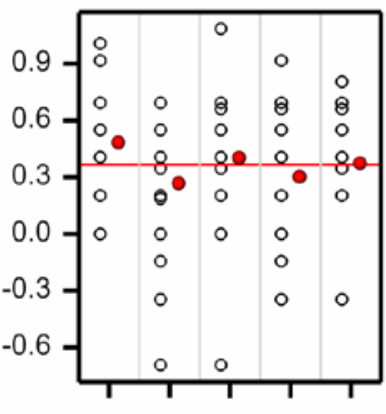

Con 11- 33- 11+ 33+

Male Eosinophils-06

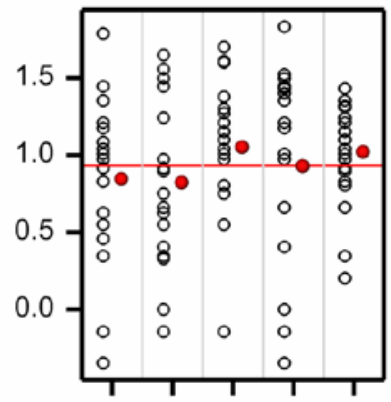

Con 11- 33- 11+ 33+
Male Lymphocytes-12

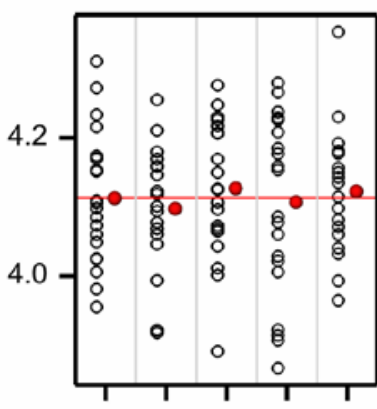

Con 11- 33- 11+ 33+

Male Neutrophils-12

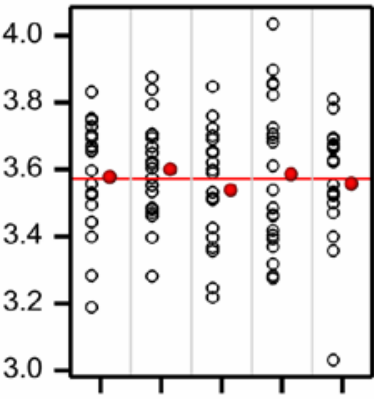

Con 11- 33- 11+ 33+

Male Monocytes-12

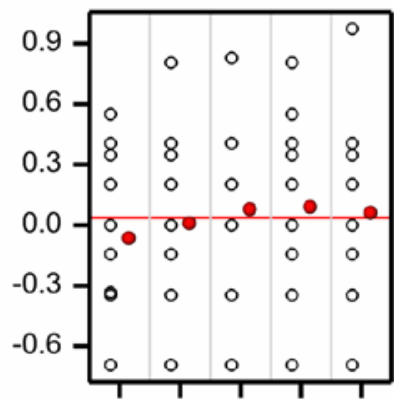

Con 11- 33- 11+ 33+

Male Eosinophils-12

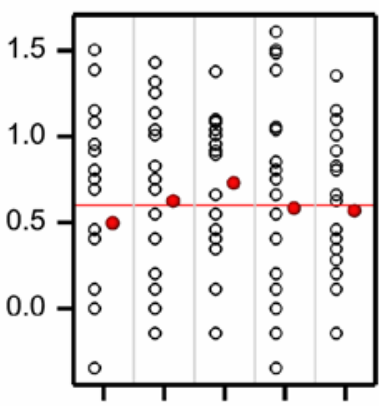

Con 11- 33- 11+ 33+
Male Lymphocytes-24

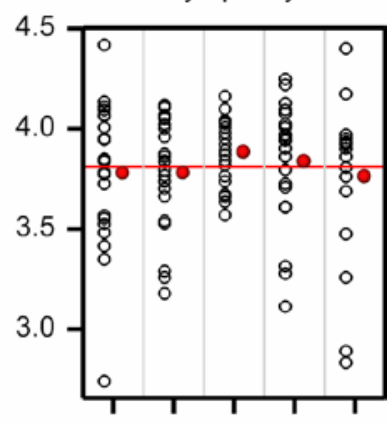

Con 11- 33- 11+33+

Male Neutrophils-24

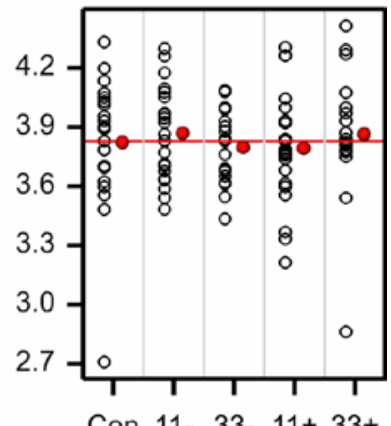

Male Monocytes-24

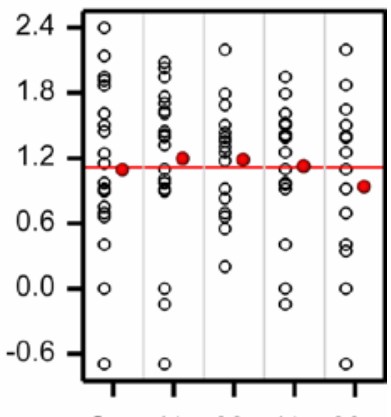

Con 11- 33- 11+ 33+

Male Eosinophils-24

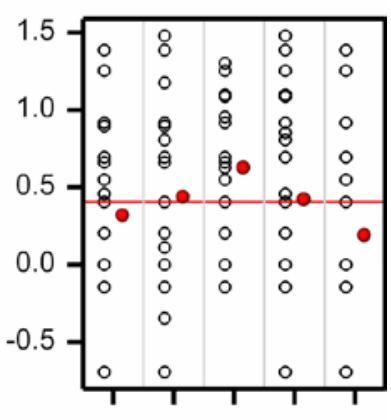

Con 11- 33- 11+ 33+ 


\section{Appendix 2. Graphs of cage means on the log scale for study A(continued)}

Red symbols denote means for feeding groups while the red line denotes the overall mean. Note that observations might overlap.

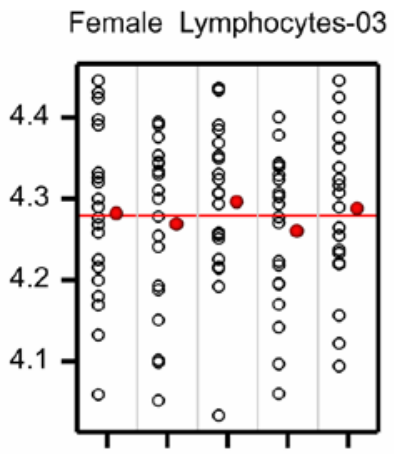

Con 11- 33- 11+ 33+

Female Neutrophils-03

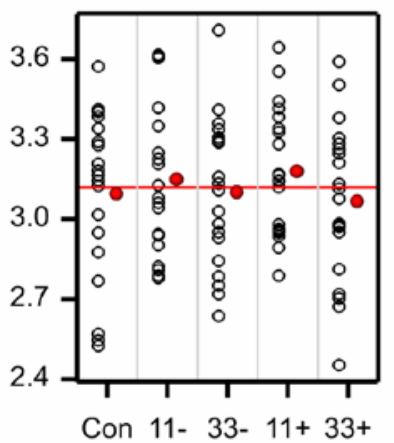

Female Monocytes-03

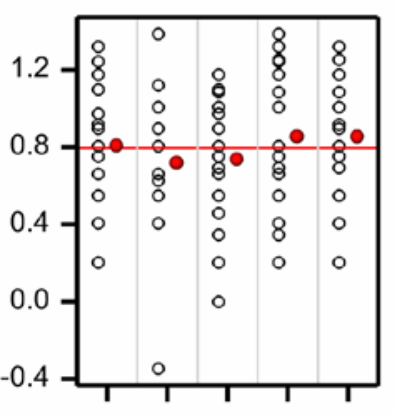

Con 11- 33- 11+ 33+

Female Eosinophils-03

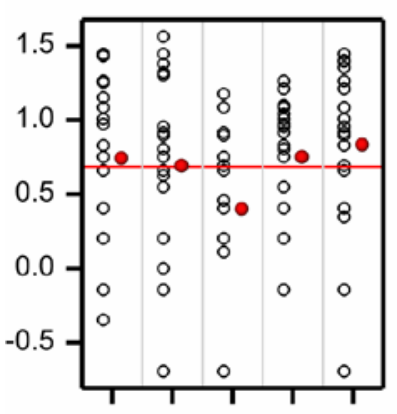

Con 11- 33- 11+ 33+
Female Lymphocytes-06

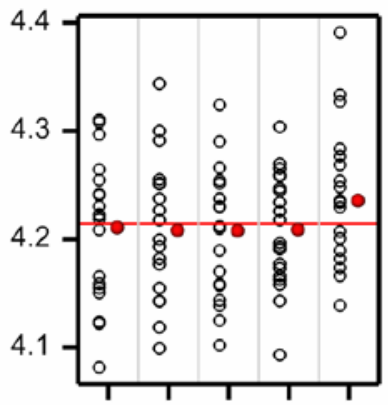

Con 11- 33- 11+ 33+

Female Neutrophils-06

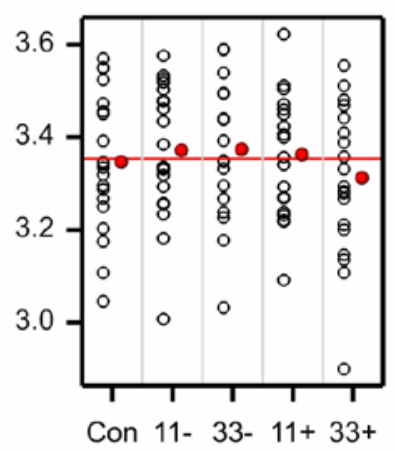

Female Monocytes-06

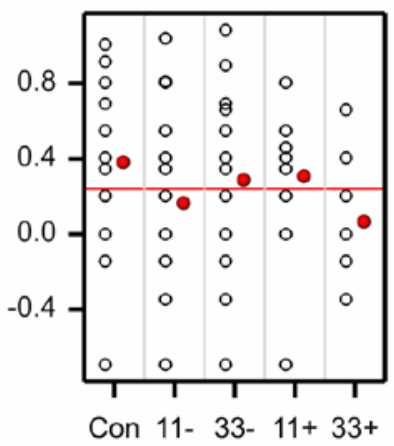

Female Eosinophils-06

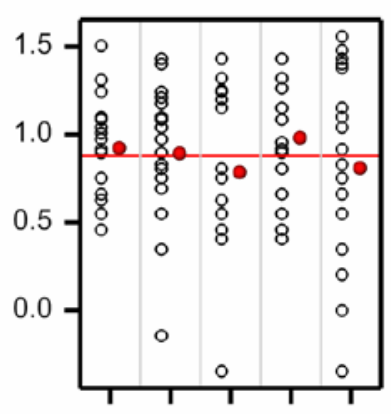

Con 11- 33- 11+ 33+
Female Lymphocytes-12

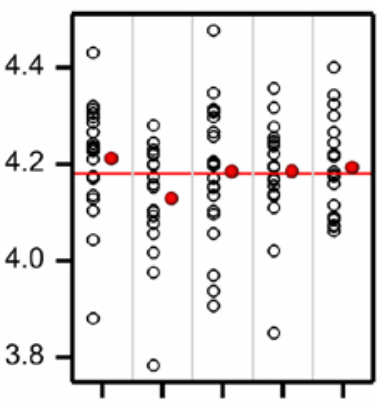

Con 11- 33- 11+ 33+

Female Neutrophils-12

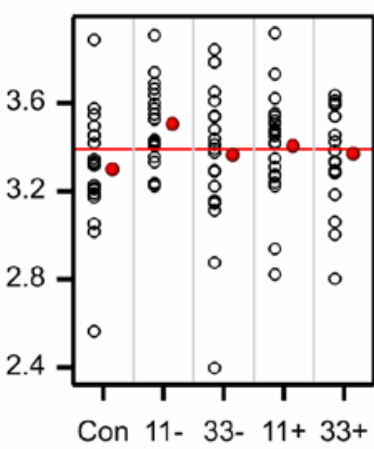

Female Monocytes-12

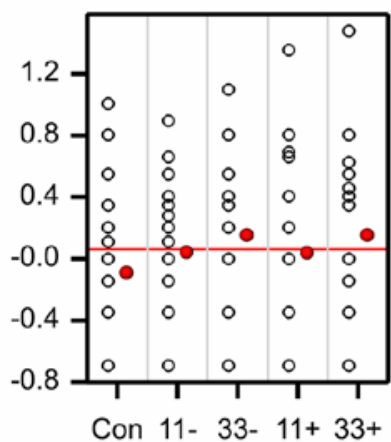

Female Eosinophils-12

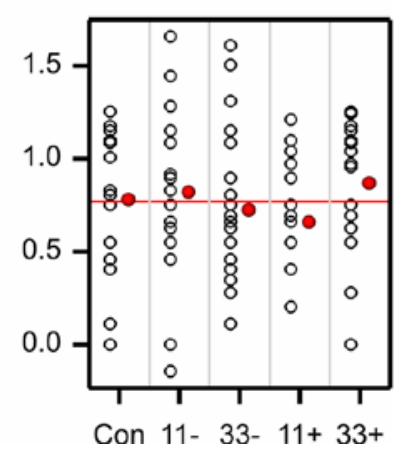

Female Lymphocytes-24

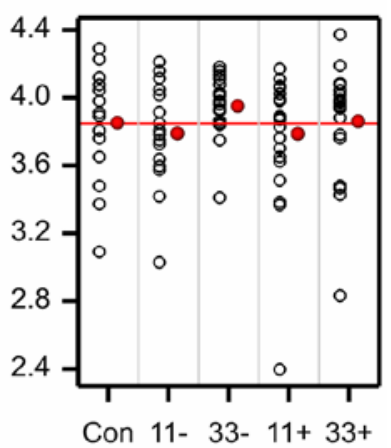

Female Neutrophils-24

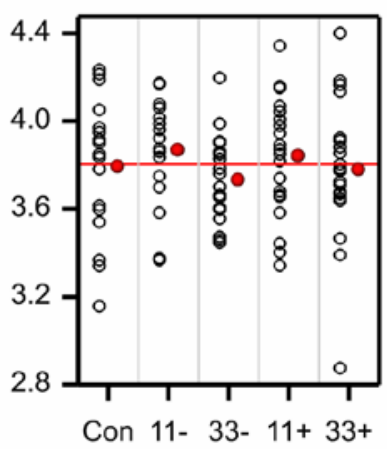

Female Monocytes-24

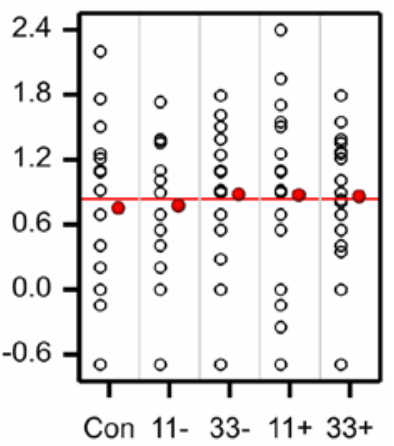

Female Eosinophils-24

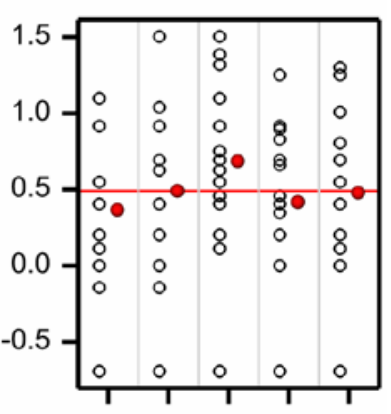

Con 11- 33- 11+ 33+ 


\section{Appendix 3. Graphs of cage means on the original scale for study $C$}

Red symbols denote means for feeding groups while the red line denotes the overall mean. Note that observations might overlap.
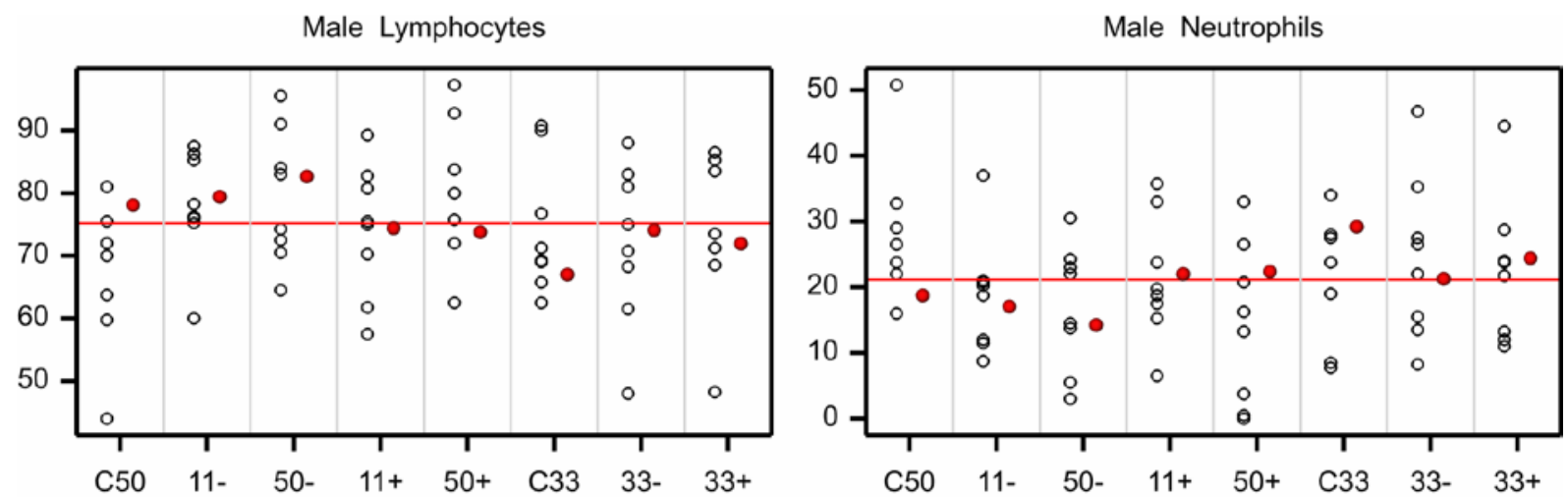

Male Monocytes
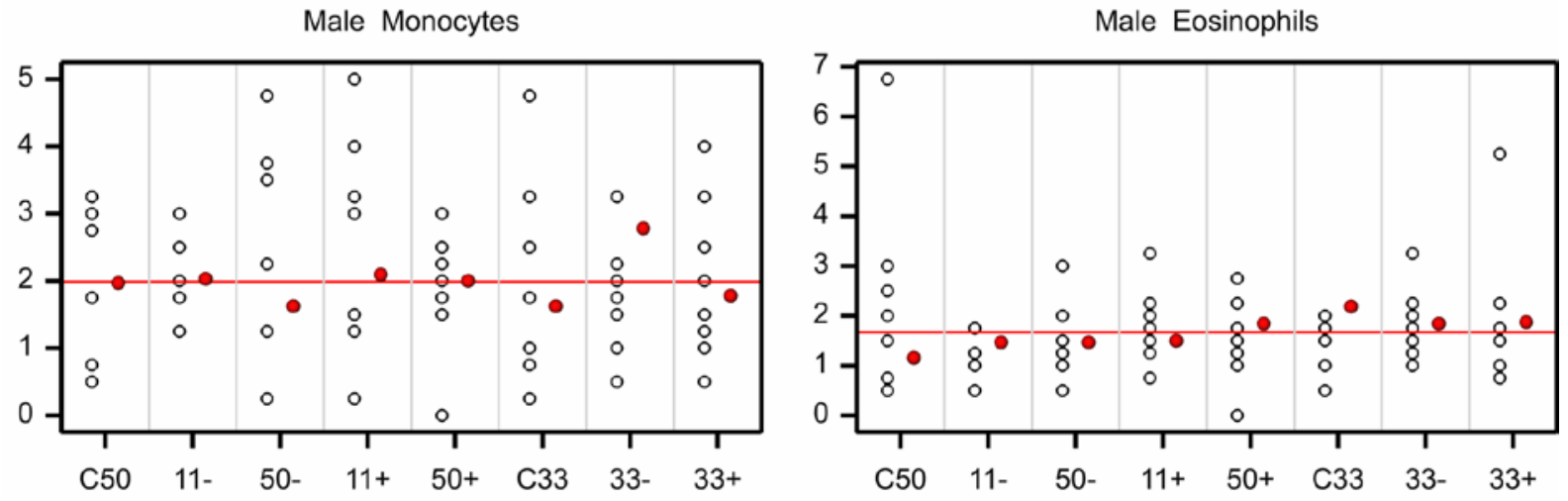

Female Lymphocytes

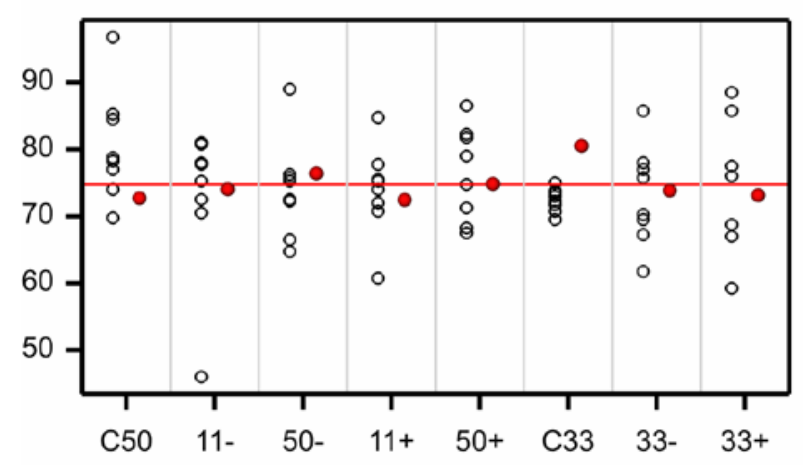

Female Neutrophils

Female Monocytes
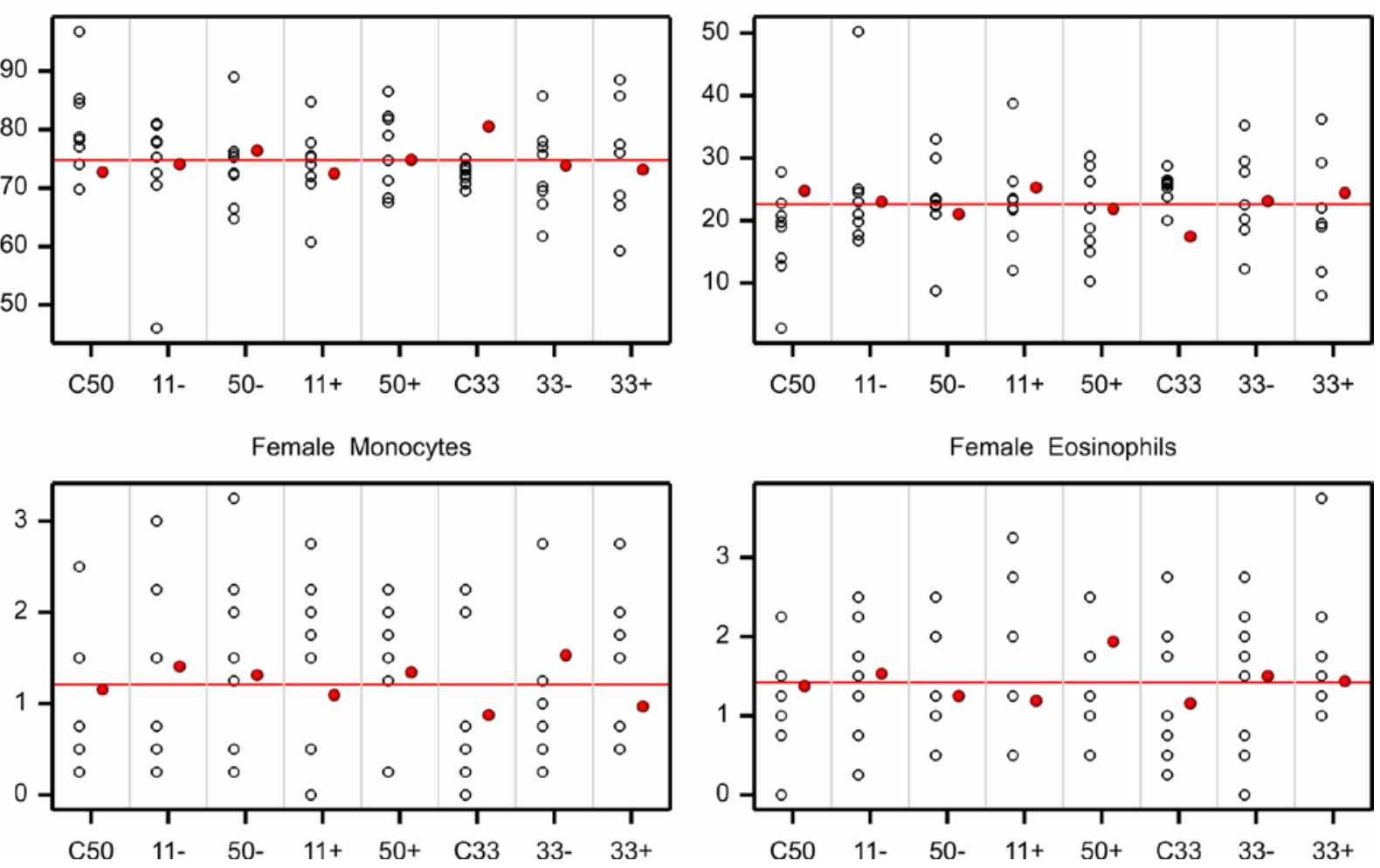

Female Eosinophils

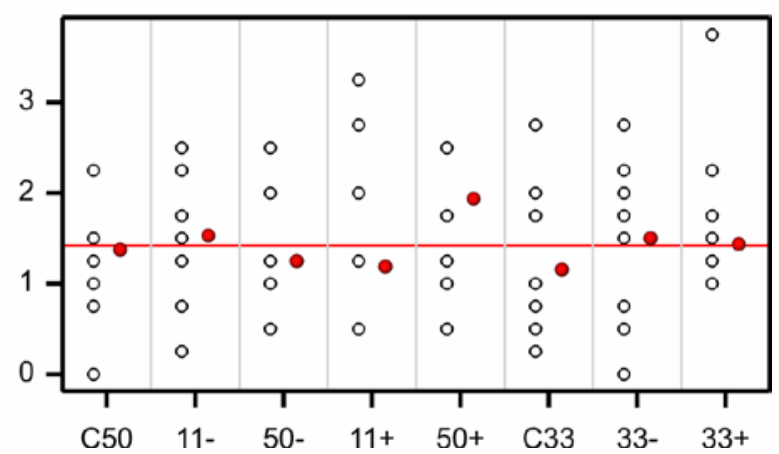




\section{Appendix 4. Graphs of cage means on the log scale for study C}

Red symbols denote means for feeding groups while the red line denotes the overall mean. Note that observations might overlap.
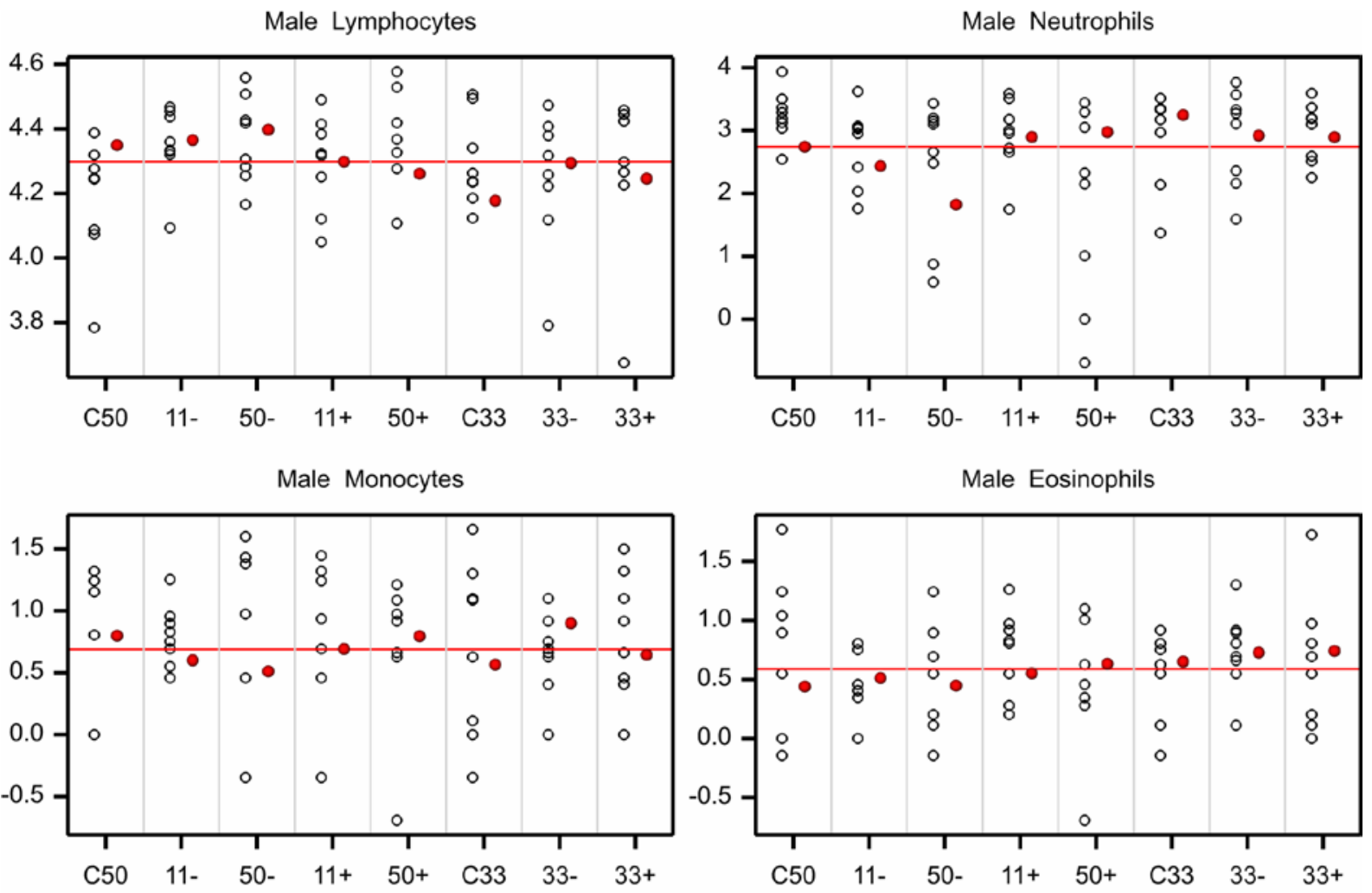

Female Lymphocytes
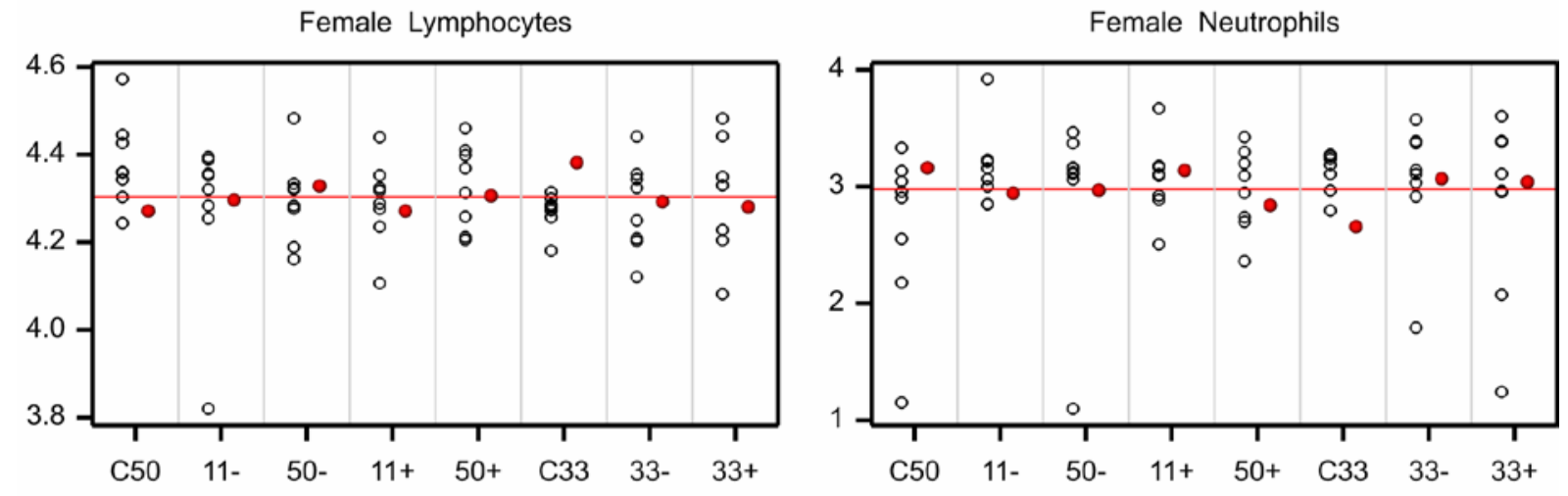

Female Monocytes

Female Eosinophils
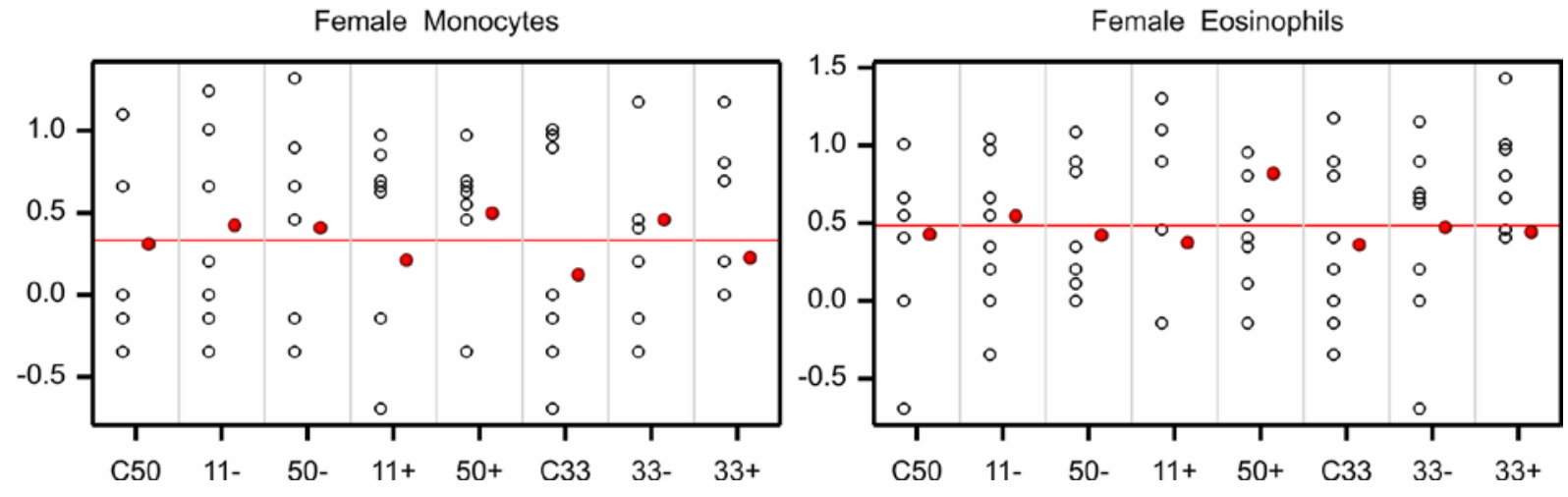
Appendix 5. P-values for difference tests for studies A and C

\begin{tabular}{|c|c|c|c|c|c|c|c|c|c|c|c|c|}
\hline \multirow{2}{*}{$\begin{array}{l}\text { A diffWBC } \\
\text { Males }\end{array}$} & \multicolumn{3}{|c|}{ NK11- } & \multicolumn{3}{|c|}{ NK33- } & \multicolumn{3}{|c|}{ NK11+ } & \multicolumn{3}{|c|}{ NK33+ } \\
\hline & Dunnet & t-test & Wilcox & Dunnet & t-test & Wilcox & Dunnet & t-test & Wilcox & Dunnet & t-test & Wilcox \\
\hline Lymphocytes-03 & 0.983 & 0.687 & 0.869 & 0.999 & 0.860 & 0.829 & 0.972 & 0.642 & 0.732 & 0.896 & 0.491 & 0.812 \\
\hline Lymphocytes-06 & 0.816 & 0.403 & 0.177 & 0.920 & 0.527 & 0.388 & 0.998 & 0.823 & 0.956 & 0.561 & 0.229 & 0.133 \\
\hline Lymphocytes-12 & 0.928 & 0.540 & 0.522 & 0.943 & 0.567 & 0.452 & 1.000 & 0.885 & 0.596 & 0.984 & 0.689 & 0.756 \\
\hline Lymphocytes-24 & 1.000 & 0.956 & 0.869 & 0.606 & 0.254 & 0.196 & 0.917 & 0.522 & 0.860 & 1.000 & 0.973 & 0.782 \\
\hline Neutrophils-03 & 1.000 & 0.902 & 0.869 & 0.993 & 0.746 & 0.869 & 1.000 & 0.994 & 0.898 & 0.999 & 0.860 & 0.784 \\
\hline Neutrophils-06 & 0.953 & 0.588 & 0.644 & 0.990 & 0.725 & 0.522 & 0.951 & 0.584 & 0.648 & 0.466 & 0.180 & 0.177 \\
\hline Neutrophils-12 & 0.968 & 0.630 & 0.784 & 0.809 & 0.397 & 0.312 & 1.000 & 0.898 & 0.701 & 0.978 & 0.664 & 0.825 \\
\hline Neutrophils-24 & 0.971 & 0.637 & 0.784 & 0.981 & 0.678 & 0.304 & 0.983 & 0.684 & 0.860 & 0.994 & 0.761 & 0.818 \\
\hline Monocytes-03 & 0.648 & 0.278 & 0.237 & 1.000 & 0.921 & 0.794 & 0.992 & 0.744 & 0.732 & 0.999 & 0.853 & 0.837 \\
\hline Monocytes-06 & 0.056 & 0.017 & 0.028 & 0.759 & 0.355 & 0.266 & 0.134 & 0.043 & 0.042 & 0.534 & 0.214 & 0.144 \\
\hline Monocytes-12 & 0.861 & 0.448 & 0.393 & 0.391 & 0.145 & 0.141 & 0.355 & 0.129 & 0.248 & 0.505 & 0.199 & 0.162 \\
\hline Monocytes-24 & 0.904 & 0.503 & 0.452 & 0.990 & 0.729 & 0.966 & 0.994 & 0.758 & 0.490 & 0.773 & 0.367 & 0.579 \\
\hline Eosinophils-03 & 0.661 & 0.287 & 0.507 & 1.000 & 0.912 & 0.794 & 0.999 & 0.838 & 0.632 & 0.858 & 0.446 & 0.641 \\
\hline Eosinophils-06 & 1.000 & 0.874 & 1.000 & 0.407 & 0.152 & 0.198 & 0.940 & 0.561 & 0.546 & 0.548 & 0.222 & 0.191 \\
\hline Eosinophils-12 & 0.799 & 0.388 & 0.260 & 0.329 & 0.118 & 0.142 & 0.821 & 0.408 & 0.926 & 0.967 & 0.626 & 0.455 \\
\hline Eosinophils-24 & 0.924 & 0.533 & 0.408 & 0.554 & 0.225 & 0.167 & 0.949 & 0.581 & 0.820 & 0.759 & 0.356 & 0.065 \\
\hline A diffWBC & \multicolumn{3}{|c|}{ NK11- } & \multicolumn{3}{|c|}{ NK33- } & \multicolumn{3}{|c|}{ NK11+ } & \multicolumn{3}{|c|}{ NK33+ } \\
\hline Females & Dunnet & t-test & Wilcox & Dunnet & t-test & Wilcox & Dunnet & t-test & Wilcox & Dunnet & t-test & Wilcox \\
\hline Lymphocytes-03 & 0.971 & 0.637 & 0.352 & 0.934 & 0.550 & 0.701 & 0.768 & 0.361 & 0.452 & 0.997 & 0.799 & 0.898 \\
\hline Lymphocytes-06 & 1.000 & 0.939 & 0.953 & 0.999 & 0.863 & 0.920 & 1.000 & 0.910 & 0.869 & 0.397 & 0.147 & 0.076 \\
\hline Lymphocytes-12 & 0.076 & 0.023 & 0.020 & 0.842 & 0.428 & 0.452 & 0.863 & 0.450 & 0.546 & 0.956 & 0.596 & 0.812 \\
\hline Lymphocytes-24 & 0.985 & 0.703 & 0.850 & 0.916 & 0.532 & 0.146 & 0.859 & 0.458 & 0.330 & 1.000 & 0.912 & 0.940 \\
\hline Neutrophils-03 & 0.872 & 0.460 & 0.293 & 1.000 & 0.929 & 0.985 & 0.499 & 0.195 & 0.245 & 0.976 & 0.655 & 0.701 \\
\hline Neutrophils-06 & 0.992 & 0.740 & 0.595 & 0.910 & 0.511 & 0.430 & 0.987 & 0.706 & 0.701 & 0.816 & 0.403 & 0.277 \\
\hline Neutrophils-12 & 0.038 & 0.011 & 0.011 & 0.812 & 0.399 & 0.388 & 0.448 & 0.171 & 0.090 & 0.760 & 0.355 & 0.475 \\
\hline Neutrophils-24 & 0.919 & 0.537 & 0.791 & 0.893 & 0.499 & 0.244 & 0.950 & 0.593 & 0.229 & 0.996 & 0.786 & 0.860 \\
\hline Monocytes-03 & 0.674 & 0.294 & 0.309 & 0.788 & 0.378 & 0.629 & 0.940 & 0.561 & 0.508 & 0.943 & 0.566 & 0.575 \\
\hline Monocytes-06 & 0.222 & 0.075 & 0.084 & 0.813 & 0.400 & 0.681 & 0.907 & 0.506 & 0.260 & 0.020 & 0.006 & 0.003 \\
\hline Monocytes-12 & 0.843 & 0.429 & 0.451 & 0.298 & 0.105 & 0.182 & 0.602 & 0.250 & 0.164 & 0.180 & 0.059 & 0.145 \\
\hline Monocytes-24 & 0.954 & 0.602 & 0.733 & 0.692 & 0.317 & 0.273 & 0.772 & 0.377 & 0.639 & 0.982 & 0.689 & 0.834 \\
\hline
\end{tabular}




\begin{tabular}{|l|l|l|l|l|l|l|l|l|l|l|l|l|}
\hline Eosinophils-03 & 0.999 & 0.866 & 0.965 & 0.171 & 0.056 & 0.153 & 1.000 & 0.962 & 1.000 & 0.961 & 0.608 & 0.601 \\
\hline Eosinophils-06 & 1.000 & 0.948 & 1.000 & 0.652 & 0.281 & 0.235 & 0.974 & 0.648 & 0.481 & 0.776 & 0.367 & 0.588 \\
\hline Eosinophils-12 & 0.944 & 0.569 & 0.571 & 0.968 & 0.630 & 0.644 & 0.533 & 0.213 & 0.107 & 0.843 & 0.429 & 0.360 \\
\hline Eosinophils-24 & 0.750 & 0.360 & 0.193 & 0.101 & 0.033 & 0.216 & 0.999 & 0.843 & 0.691 & 0.894 & 0.499 & 0.589 \\
\hline
\end{tabular}

\begin{tabular}{|c|c|c|c|c|c|c|c|c|c|c|c|c|}
\hline C: diffWBC & \multicolumn{3}{|c|}{ NK11-/50 vs Con50 } & \multicolumn{3}{|c|}{ NK50- vs Con50 } & \multicolumn{3}{|c|}{ NK11+/50 vs Con50 } & \multicolumn{3}{|c|}{ NK50+ vs Con50 } \\
\hline Males & Dunnet & t-test & Wilcox & Dunnet & t-test & Wilcox & Dunnet & t-test & Wilcox & Dunnet & t-test & Wilcox \\
\hline Lymphocytes & 0.998 & 0.818 & 0.641 & 0.884 & 0.478 & 0.250 & 0.856 & 0.444 & 0.547 & 0.486 & 0.191 & 0.250 \\
\hline Neutrophils & 0.825 & 0.413 & 0.547 & 0.055 & 0.016 & 0.148 & 0.981 & 0.676 & 0.742 & 0.922 & 0.531 & 0.547 \\
\hline Monocytes & 0.777 & 0.371 & 0.547 & 0.497 & 0.196 & 0.383 & 0.968 & 0.629 & 0.844 & 1.000 & 0.987 & 0.945 \\
\hline Eosinophils & 0.993 & 0.754 & 0.844 & 1.000 & 0.973 & 0.933 & 0.967 & 0.628 & 0.742 & 0.820 & 0.409 & 0.641 \\
\hline C: diffWBC & \multicolumn{3}{|c|}{ NK11-/50 vs Con50 } & \multicolumn{3}{|c|}{ NK50- vs Con50 } & \multicolumn{3}{|c|}{ NK11+/50 vs Con50 } & \multicolumn{3}{|c|}{ NK50+ vs Con50 } \\
\hline Females & Dunnet & t-test & Wilcox & Dunnet & t-test & Wilcox & Dunnet & t-test & Wilcox & Dunnet & t-test & Wilcox \\
\hline Lymphocytes & 0.977 & 0.661 & 0.742 & 0.712 & 0.322 & 0.742 & 1.000 & 0.999 & 0.250 & 0.931 & 0.546 & 0.547 \\
\hline Neutrophils & 0.848 & 0.436 & 1.000 & 0.896 & 0.493 & 0.547 & 1.000 & 0.935 & 1.000 & 0.600 & 0.251 & 0.383 \\
\hline Monocytes & 0.968 & 0.632 & 0.933 & 0.981 & 0.679 & 0.553 & 0.982 & 0.680 & 0.383 & 0.846 & 0.434 & 0.461 \\
\hline Eosinophils & 0.971 & 0.639 & 0.528 & 1.000 & 0.980 & 0.844 & 0.998 & 0.829 & 0.674 & 0.341 & 0.124 & 0.195 \\
\hline
\end{tabular}

\begin{tabular}{|c|c|c|c|c|c|c|c|c|c|c|c|c|c|c|c|}
\hline C: diffWBC & \multicolumn{3}{|c|}{ Con50 vs Con33 } & \multicolumn{3}{|c|}{ NK50- vs NK33- } & \multicolumn{3}{|c|}{ NK50+ vs NK33+ } & \multicolumn{3}{|c|}{ NK33- vs Con33 } & \multicolumn{3}{|c|}{ NK33+ vs Con33 } \\
\hline Males & Dunn & t-test & Wlcx & Dunn & t-test & Wlcx & Dunn & t-test & Wlcx & Dunn & t-test & Wlcx & Dunn & t-test & Wlcr \\
\hline Lymphocytes & & 0.013 & 0.008 & & 0.127 & 0.055 & & 0.818 & 0.945 & 0.154 & 0.087 & 0.195 & 0.493 & 0.312 & 0.742 \\
\hline Neutrophils & & 0.178 & 0.078 & & 0.005 & 0.055 & & 0.828 & 0.844 & 0.583 & 0.381 & 0.313 & 0.536 & 0.344 & 0.547 \\
\hline Monocytes & & 0.292 & 0.250 & & 0.083 & 0.109 & & 0.496 & 0.735 & 0.229 & 0.134 & 0.313 & 0.909 & 0.719 & 0.800 \\
\hline Eosinophils & & 0.365 & 0.674 & & 0.232 & 0.547 & & 0.639 & 0.624 & 0.924 & 0.744 & 0.844 & 0.895 & 0.697 & 1.000 \\
\hline C: diffWBC & \multicolumn{3}{|c|}{ Con50 vs Con33 } & \multicolumn{3}{|c|}{ NK50- vs NK33- } & \multicolumn{3}{|c|}{ NK50+ vs NK33+ } & \multicolumn{3}{|c|}{ NK33- vs Con33 } & \multicolumn{3}{|c|}{ NK33+ vs Con33 } \\
\hline Females & Dunn & t-test & Wlcx & Dunn & t-test & Wlcx & Dunn & t-test & WIcx & Dunn & t-test & Wlcx & Dunn & t-test & WICx \\
\hline Lymphocytes & & 0.058 & 0.250 & & 0.534 & 0.148 & & 0.659 & 0.641 & 0.214 & 0.124 & 0.109 & 0.145 & 0.082 & 0.195 \\
\hline Neutrophils & & 0.074 & 0.109 & & 0.728 & 0.461 & & 0.471 & 0.742 & 0.246 & 0.144 & 0.250 & 0.288 & 0.171 & 0.383 \\
\hline Monocytes & & 0.433 & 0.461 & & 0.836 & 0.844 & & 0.259 & 0.195 & 0.276 & 0.164 & 0.148 & 0.871 & 0.664 & 0.834 \\
\hline Eosinophils & & 0.787 & 0.461 & & 0.840 & 0.844 & & 0.137 & 0.078 & 0.864 & 0.655 & 0.726 & 0.925 & 0.745 & 0.834 \\
\hline
\end{tabular}




\section{Appendix 6. Tests for normality and homogeneity of variance for studies A and C}

P-values for the Shapiro-Wilks (SW) normality test are given separately for each feeding group, and also for the ANOVA residuals. In addition P-values for Bartlett and Levene test for homogeneity of variance are given. P-values smaller than $0.01 / 0.05$ have a gold/yellow background.

\begin{tabular}{|l|c|c|c|c|c|c|c|c|}
\hline Study A: Male diffWBC & SW Control & SW NK11- & SW NK33- & SW NK11+ & SW NK33+ & SW residual & Bartlett & Levene \\
\hline Lymphocytes-03 & 0.548 & 0.096 & 0.998 & 0.430 & 0.266 & 0.155 & 0.019 & 0.013 \\
\hline Lymphocytes-06 & 0.561 & 0.284 & 0.509 & 0.066 & 0.925 & 0.199 & 0.268 & 0.713 \\
\hline Lymphocytes-12 & 0.879 & 0.502 & 0.567 & 0.089 & 0.575 & 0.754 & 0.366 & 0.072 \\
\hline Lymphocytes-24 & 0.177 & 0.044 & 0.622 & 0.043 & 0.003 & 0.077 & 0.004 & 0.116 \\
\hline Neutrophils-03 & 0.648 & 0.404 & 0.189 & 0.735 & 0.629 & 0.639 & 0.077 & 0.058 \\
\hline Neutrophils-06 & 0.715 & 0.007 & 0.272 & 0.668 & 0.728 & 0.867 & 0.790 & 0.962 \\
\hline Neutrophils-12 & 0.323 & 0.989 & 0.736 & 0.271 & 0.028 & 0.917 & 0.450 & 0.108 \\
\hline Neutrophils-24 & 0.010 & 0.805 & 0.583 & 0.500 & 0.005 & 0.006 & 0.050 & 0.724 \\
\hline Monocytes-03 & 0.116 & 0.528 & 0.202 & 0.045 & 0.074 & 0.025 & 0.178 & 0.196 \\
\hline Monocytes-06 & 0.394 & 0.021 & 0.031 & 0.087 & 0.050 & 0.633 & 0.409 & 0.343 \\
\hline Monocytes-12 & 0.050 & 0.486 & 0.224 & 0.504 & 0.178 & 0.888 & 0.756 & 0.500 \\
\hline Monocytes-24 & 0.885 & 0.011 & 0.872 & 0.068 & 0.332 & 0.202 & 0.066 & 0.105 \\
\hline Eosinophils-03 & 0.788 & 0.958 & 0.139 & 0.260 & 0.796 & 0.009 & 0.938 & 0.899 \\
\hline Eosinophils-06 & 0.392 & 0.433 & 0.198 & 0.108 & 0.041 & 0.076 & 0.110 & 0.056 \\
\hline Eosinophils-12 & 0.286 & 0.571 & 0.164 & 0.301 & 0.707 & 0.974 & 0.263 & 0.111 \\
\hline Eosinophils-24 & 0.467 & 0.476 & 0.203 & 0.241 & 0.207 & 0.139 & 0.505 & 0.430 \\
\hline Study A: Female diffWBC & SW Control & SW NK11- & SW NK33- & SW NK11+ & SW NK33+ & SW residual & Bartlett & Levene \\
\hline Lymphocytes-03 & 0.844 & 0.046 & 0.262 & 0.350 & 0.818 & 0.002 & 0.981 & 0.939 \\
\hline Lymphocytes-06 & 0.493 & 0.902 & 0.851 & 0.869 & 0.371 & 0.336 & 0.878 & 0.913 \\
\hline Lymphocytes-12 & 0.164 & 0.027 & 0.756 & 0.016 & 0.311 & 0.013 & 0.585 & 0.630 \\
\hline Lymphocytes-24 & 0.425 & 0.358 & 0.018 & 0.000 & 0.003 & 0.018 & 0.018 & 0.214 \\
\hline Neutrophils-03 & 0.117 & 0.139 & 0.663 & 0.587 & 0.949 & 0.121 & 0.862 & 0.877 \\
\hline Neutrophils-06 & 0.657 & 0.258 & 0.734 & 0.794 & 0.476 & 0.430 & 0.911 & 0.948 \\
\hline Neutrophils-12 & 0.193 & 0.705 & 0.096 & 0.304 & 0.077 & 0.222 & 0.133 & 0.476 \\
\hline Neutrophils-24 & 0.490 & 0.093 & 0.680 & 0.850 & 0.079 & 0.565 & 0.228 & 0.492 \\
\hline
\end{tabular}




\begin{tabular}{|c|c|c|c|c|c|c|c|c|}
\hline Monocytes-03 & 0.576 & 0.095 & 0.261 & 0.339 & 0.759 & 0.043 & 0.838 & 0.707 \\
\hline Monocytes-06 & 0.325 & 0.609 & 0.776 & 0.002 & 0.049 & 0.297 & 0.200 & 0.077 \\
\hline Monocytes-12 & 0.085 & 0.700 & 0.363 & 0.126 & 0.403 & 0.092 & 0.748 & 0.679 \\
\hline Monocytes-24 & 0.986 & 0.563 & 0.265 & 0.426 & 0.259 & 0.780 & 0.619 & 0.507 \\
\hline Eosinophils-03 & 0.049 & 0.281 & 0.029 & 0.014 & 0.014 & 0.000 & 0.581 & 0.709 \\
\hline Eosinophils-06 & 0.719 & 0.174 & 0.032 & 0.301 & 0.594 & 0.140 & 0.044 & 0.053 \\
\hline Eosinophils-12 & 0.133 & 0.302 & 0.333 & 0.414 & 0.019 & 0.377 & 0.436 & 0.479 \\
\hline Eosinophils-24 & 0.214 & 0.784 & 0.172 & 0.076 & 0.184 & 0.785 & 0.992 & 0.966 \\
\hline
\end{tabular}

\begin{tabular}{|c|c|c|c|c|c|c|c|c|c|c|c|}
\hline \multicolumn{12}{|l|}{ C: diffWBC } \\
\hline Males & Con50 & NK11-/50 & NK50- & NK11+/50 & NK50+ & Con33 & NK33- & NK33+ & Residual & Bartlett & Levene \\
\hline Lymphocytes & 0.071 & 0.846 & 0.541 & 0.281 & 0.005 & 0.198 & 0.737 & 0.197 & 0.033 & 0.500 & 0.887 \\
\hline Neutrophils & 0.429 & 0.029 & 0.300 & 0.016 & 0.518 & 0.875 & 0.386 & 0.386 & 0.409 & 0.006 & 0.001 \\
\hline Monocytes & 0.926 & 0.065 & 0.015 & 0.647 & 0.938 & 0.012 & 0.136 & 0.632 & 0.077 & 0.064 & 0.003 \\
\hline Eosinophils & 0.333 & 0.966 & 0.193 & 0.087 & 0.486 & 0.438 & 0.678 & 0.794 & 0.138 & 0.194 & 0.145 \\
\hline \multicolumn{12}{|l|}{ C: diffWBC } \\
\hline Females & Con50 & NK11-/50 & NK50- & NK11+/50 & NK50+ & Con33 & NK33- & NK33+ & Residual & Bartlett & Levene \\
\hline Lymphocytes & 0.001 & 0.480 & 0.498 & 0.044 & 0.830 & 0.745 & 0.721 & 0.915 & 0.063 & 0.045 & 0.387 \\
\hline Neutrophils & 0.036 & 0.000 & 0.859 & 0.044 & 0.077 & 0.072 & 0.538 & 0.022 & 0.001 & 0.004 & 0.164 \\
\hline Monocytes & 0.283 & 0.310 & 0.040 & 0.172 & 0.321 & 0.024 & 0.018 & 0.406 & 0.672 & 0.929 & 0.613 \\
\hline Eosinophils & 0.898 & 0.189 & 0.924 & 0.766 & 0.688 & 0.215 & 0.173 & 0.458 & 0.321 & 0.762 & 0.536 \\
\hline
\end{tabular}

